\title{
Sporopollenin-inspired design and synthesis of robust materials
}

\section{Authors:}

Christopher M. Glinkerman, ${ }^{1}$ Shaoting Lin, ${ }^{2}$ Jiahua Ni, ${ }^{2}$ Fu-Shuang Li, ${ }^{1}$ Xuanhe Zhao, ${ }^{2}$ Jing-Ke Weng ${ }^{1,3 *}$

\section{Affiliations:}

${ }^{1}$ Whitehead Institute for Biomedical Research, Cambridge, Massachusetts 02142, United States.

${ }^{2}$ Department of Mechanical Engineering, Massachusetts Institute of Technology, Cambridge, Massachusetts 02139, United States.

${ }^{3}$ Department of Biology, Massachusetts Institute of Technology, Cambridge, Massachusetts 02139, United States.

*Correspondence author, Email: wengj@wi.mit.edu, Tel.: +1 6173244921 


\begin{abstract}
Sporopollenin is a mechanically robust and chemically inert biopolymer that constitutes the outer protective exine layer of plant spores and pollen grains. Recent investigation of the molecular structure of pine sporopollenin revealed unique monomeric units and inter-unit linkages distinct from other previously known biopolymers, which could be harnessed for new material design. Here, we report the bioinspired synthesis of a series of sporopollenin analogues. This exercise confirms large portions of the previously proposed pine sporopollenin structural model, while the measured chemical, thermal, and mechanical properties of the synthetic sporopollenins indicate favorable attributes of a new kind of robust material. This study explores a new design framework of robust materials inspired by natural sporopollenins, and provides insights and reagents for future elucidation and engineering of sporopollenin biosynthesis in plants.
\end{abstract}

One Sentence Summary: Biopolymer-inspired synthesis of sporopollenin analogues validates prior pine sporopollenin structural hypothesis and illustrates a new design system for robust materials. 
Sporopollenin is the general designation for a class of chemically related and ubiquitous biopolymers that comprise the exine of plant spores and pollen grains (Figure 1A) (1). In its natural role, sporopollenin serves to protect the fragile gametes of land plants against myriad environmental insults including desiccation, ultraviolet irradiation, chemical degradation, and mechanical stress (1). As a result, sporopollenin has evolved to be one of the strongest and most chemically resistant known materials of direct biological origin boasting elastic moduli as high as $16 \pm 2.5 \mathrm{GPa}(2)$, resistance to hydrostatic pressures in excess of $10 \mathrm{GPa}(3)$, and inertness towards a wide variety of organic solvents (4). These properties have led to the successful application of natural sporopollenin to chromatography (5), solid phase peptide synthesis (6), catalyst solid supports (7), magnetic nanoparticle synthesis (8), and the encapsulation of enzymes (9), pharmaceuticals (10), and whole cells $(10,11)$. Despite these early successes, the implementation of sporopollenin-based technologies has been stymied by a historically limited understanding of the molecular structure of sporopollenin and lack of consistent supply of naturederived sporopollenin materials.

For over 200 years, the chemical community has painstakingly engaged in structural elucidation studies of plant sporopollenins spanning myriad degradative, spectroscopic, and spectrometric methodologies with limited progress (1). Facilitated by the implementation of ${ }^{13} \mathrm{C}$ magic angle spinning solid state nuclear magnetic resonance (MAS ssNMR) spectroscopy and degradative thioacidolysis, we recently reported the most detailed hypothesis for the molecular structure of Pinus rigida sporopollenin to date (Figure 1B) (12). Our study suggests that $P$. rigida sporopollenin is principally comprised of aliphatic polyketide-derived polyvinyl alcohol units crosslinked by $p$-coumaryl-substituted fatty acid-derived $\mathrm{C} 16$ aliphatic units via acetal linkages (Figure 1C) (12). This proposed structure has withstood recent spectroscopic scrutiny (13) and, therefore, represents a validated starting point for synthesis of structural analogues that retain sporopollenin's fascinating properties. Here, we aim to further demystify one of Nature's most robust materials and facilitate its potential application through the bioinspired synthesis of sporopollenin.

We envisioned the bioinspired synthetic sporopollenin analogues to arise from the wellprecedented acid-catalyzed crosslinking of commercial polyvinyl alcohol $(14,15)$ with a suite of synthetically accessed $\alpha, \omega$-dialdehydes (16). The central feature of this approach is the divergent preparation of variously substituted $\alpha, \omega$-dialdehyde crosslinkers via either oxidation of simple $\alpha, \omega$ alkanols or an alkylation/ring expansion sequence that permits variability of chain length, substituent identity/position, and chirality. Such an approach would permit the rapid and divergent preparation of myriad sporopollenin-like polymers with discrete control over linker length, linker 
substituent(s), degree of crosslinking, and polyvinyl alcohol backbone properties, including average molecular weight, polydispersity, and tacticity.

We crosslinked a series of unsubstituted $\alpha, \omega$-dialdehydes (1-5), prepared by oxidation of the corresponding commercially available $\alpha, \omega$-alkanols (S1-S5) (16), with $5 \%$ polyvinyl alcohol (PVA) in DMSO under acid-catalyzed conditions (Figure 2A) (14). While a temperature of $\geq 55^{\circ} \mathrm{C}$ was required for efficient crosslinking, extended periods of drying at $\geq 55{ }^{\circ} \mathrm{C}$ resulted in pronounced discoloration of the crosslinked material. This is likely due to the known acidcatalyzed decomposition of DMSO at elevated temperature (17). In order to minimize the impact of this undesired side-reaction, polymers were cured at $55-60^{\circ} \mathrm{C}$ and 760 torr for $2 \mathrm{~h}$ under air, then dried at $40{ }^{\circ} \mathrm{C}$ and 250 torr for $72 \mathrm{~h}$ under air resulting in nearly colorless, transparent crosslinked polymers. With an efficient synthetic methodology in hand, a suite of simplified sporopollenin analogues with theoretical degrees of crosslinking ranging from 5-50\% (Table S1), crosslinker length ranging from $\mathrm{C} 8-\mathrm{C} 16$, and PVA average molecular weight ranging from 31,000$186,000 \mathrm{~g} / \mathrm{mol}$ were prepared for physical, thermal, and chemical evaluation, totaling 13 discrete analogues (6-18, see Table S2 for numbering convention).

While these simplified sporopollenin analogues facilitated the optimization of the aforementioned curing method and the rapid establishment of preliminary structure-property relationships, we sought to more closely reproduce the authentic sporopollenin linker to both test the validity of the prior $P$. rigida sporopollenin structural model (12) and provide precedent for the divergent preparation of myriad functionalized linkers bearing substituents both natural and previously unobserved in sporopollenin across plant families (Figure 2B). Accordingly, alkylation of cyclohexylimine 19 (18) with bromoolefin 20 ( ${ }^{n} \mathrm{BuLi}$, DIPA, THF, 0-23 ${ }^{\circ} \mathrm{C}, 22 \mathrm{~h}, 69 \%$ ) provided, after subsequent oxidative cleavage $\left(\mathrm{NaIO}_{4}, \mathrm{KMnO}_{4}\right.$, acetone $\left./ \mathrm{H}_{2} \mathrm{O}, 23{ }^{\circ} \mathrm{C}, 20 \mathrm{~h}, 81 \%\right)$ and alkylation ( $\mathrm{Mel}, \mathrm{K}_{2} \mathrm{CO}_{3}$, acetone, reflux, 24 h, 89\%), ester 21. Ring expansion under BaeyerVilliger conditions (19) afforded the corresponding caprolactone $22\left(m \mathrm{CPBA}, \mathrm{CH}_{2} \mathrm{Cl}_{2}, 0-23{ }^{\circ} \mathrm{C}\right.$, $20 \mathrm{~h}, 91 \%$ ) which underwent smooth one-pot ring opening and esterification to afford diester 23 ( $\mathrm{NaOMe}, \mathrm{MeOH}, 23^{\circ} \mathrm{C}, 3.5 \mathrm{~h}, 85 \%$ ). This simultaneously exposed a key alcohol moiety for further functionalization. Steglich esterification (20) with TBS-protected p-coumaric acid (S8) (21) provided triester 24 (S8, DMAP, DCC, $\mathrm{CH}_{2} \mathrm{Cl}_{2}, 23-40{ }^{\circ} \mathrm{C}, 20 \mathrm{~h}, 69 \%$ ), which, after reduction $\left(\mathrm{LiBH}_{4}, \mathrm{Et}_{2} \mathrm{O}, 23{ }^{\circ} \mathrm{C}, 3 \mathrm{~h}, 64 \%\right.$ ), deprotection (TBAF, THF, $23{ }^{\circ} \mathrm{C}, 5 \mathrm{~min}, 90 \%$ ), and oxidation $\left(\mathrm{SO}_{3} \cdot \mathrm{py}, \mathrm{Et}_{3} \mathrm{~N}, \mathrm{DMSO} / \mathrm{CH}_{2} \mathrm{Cl}_{2}, 0{ }^{\circ} \mathrm{C}, 2.5 \mathrm{~h}, 58 \%\right)$, was converted to the target dialdehyde 25 (9 steps from commercial, $9 \%$ overall yield, see Figures $\mathbf{S 1 - S 1 0 ~ f o r ~ s p e c t r a ) . ~ I t ~ i s ~ n o t a b l e ~ t h a t ~ m a n y ~}$ intermediates en route to dialdehyde 25 (e.g. 23, 24) are closely related to proposed biosynthetic pathway intermediates and, thus, may be implemented in the future validation of sporopollenin 
biosynthetic hypotheses. While yet unexplored, we anticipate this synthetic route will permit rapid divergence to myriad sporopollenin linker analogues in the future (Figure 2C). Analogous to the simple $\alpha, \omega$-dialdehyde crosslinkers (16), synthetically accessed dialdehyde $\mathbf{2 5}$ was crosslinked with PVA (14) to afford synthetic linker sporopollenin analogue 26 (Figure 3A), representing the closest synthetic recapitulation of $P$. rigida sporopollenin disclosed to date (12).

Initial comparison of synthetic analogues 15 and 26 with authentic $P$. rigida sporopollenin revealed a high degree of similarity in both ${ }^{13} \mathrm{C}$ MAS ssNMR $(22,23)$ (Figure 3B) and attenuated total reflectance Fourier-transform infrared (ATR-FTIR, Figure 3C) spectroscopies (Figures S11-18) $(13,24)$. The presence of acetal cross-linkages in all three samples was confirmed by a broad NMR signal from 97-103 ppm corroborated by an IR band at $\sim 1100 \mathrm{~cm}^{-1}$ corresponding to an asymmetric stretching of aliphatic ethers. The broadness of both signals coupled with the fine structure of the IR band suggest the acetals are present in a variety of subtly distinct chemical environments as anticipated in a highly crosslinked, three-dimensional polymeric structure. Further mutual upfield NMR signals from 62-80 ppm and 20-52 ppm were consistent with oxygen-bearing and aliphatic methylene carbons, respectively, attributed to the skipped polyol backbone and aliphatic crosslinkers. The latter is further corroborated by the presence of methylene $\mathrm{C}-\mathrm{H}$ asymmetric and symmetric stretches in the IR spectra at 2920 and $2850 \mathrm{~cm}^{-1}$, respectively. More detailed comparison of synthetic linker sporopollenin analogue 26 and authentic $P$. rigida sporopollenin revealed a series of remarkably similar downfield NMR signals corresponding to the arene (111-121, 123-135, 155-162 ppm), olefin (111-121, 164-170 ppm), and carbonyl (164-170 ppm) of the $p$-coumaryl ester moiety with a sharp IR signal at $\sim 1514 \mathrm{~cm}^{-}$ ${ }^{1}$ attributed to an aromatic ring mode of the coumaryl ester (13). As chemical moieties previously predicted to be secondary to the structural linkages of sporopollenin were not included in current synthetic efforts, NMR signals at 87 and 96 ppm, diagnostic of a-pyrones, were observed only in the authentic $P$. rigida sporopollenin (12). Curiously, a medium to sharp IR signal at $\sim 830 \mathrm{~cm}^{-1}$ previously attributed in $P$. ponderosa sporopollenin to an aromatic $\mathrm{CH}$ out-of-plane bending (13) is present in all three samples, suggesting a nonaromatic origin. In addition to the highlighted signals, the IR fingerprint regions across samples demonstrate excellent overlap strongly supporting the highly similar nature of the authentic and synthetic samples.

In analogy to our prior $P$. rigida sporopollenin structural elucidation efforts, synthetic analogues 15 and 26 were submitted to degradative thioacidolysis followed by liquid chromatography-high resolution mass spectrometry (DT/LC-HRMS) analysis (12), and compared with authentic material to assess similarities in chemical reactivity (Figures 3D, S19-20). Spectrometry revealed the presence of analogous bis-thioenol ether degradation products in each 
sample, likely resulting from the in-source fragmentation of the corresponding bis-dithioacetal of the relevant crosslinking $\alpha, \omega$-dialdehyde, suggesting similar mechanisms of degradation across synthetic $(15,26)$ and authentic sporopollenins $(12)$. The chemical stability of analogue 15 was further examined by calculating mass differences in samples exposed to organic solvents over 24 $\mathrm{h}$. These solvolysis studies revealed no significant mass changes suggesting broad resistance of sporopollenin analogues to organic solvents, consistent with prior reports of the chemical resilience of natural sporopollenins (see Table S3) (4).

Taken together, the spectroscopic, spectrometric, and chemical reactivity data across synthetic sporopollenin analogues 15 and 26 bear remarkable similarities to authentic $P$. rigida sporopollenin (12), thus demonstrating, through chemical synthesis, the accuracy of large portions of our previous structural hypothesis, and providing access to both an underexplored class of bioinspired and biocompatible polymer and tools for sporopollenin biosynthetic pathway elucidation. While independent ${ }^{13} \mathrm{C}$ MAS ssNMR studies are currently unreported, the ATR-FTIR data disclosed herein are broadly consistent with recent analyses of $P$. ponderosa sporopollenin by Lutzke, et al. (13), and suggest high structural similarity between $P$. rigida and $P$. ponderosa sporopollenin.

With our structural hypothesis for $P$. rigida sporopollenin largely confirmed, we set out to examine the thermal and mechanical properties of the synthetic sporopollenin analogues. Prior studies on a variety of natural sporopollenins have revealed appealing properties including high modulus (2) and resistance to significant hydrostatic pressures (3), thus, we anticipated synthetic analogues would exhibit similar properties that would permit their wide application in materials science. To those ends, thermogravimetric analysis (TGA) was conducted on all analogues disclosed to ascertain their thermal stability and extent of contamination by low molecular weight solvents and process aids (Table S9, Figures S44-S57). Across all analogues, a series of three mass losses of $34.6 \pm 2.4 \%$ at $213 \pm 9.8^{\circ} \mathrm{C}, 54.1 \pm 4.0 \%$ at $453 \pm 5.9{ }^{\circ} \mathrm{C}$, and $9.8 \pm 1.4 \%$ at 535 $\pm 8.0^{\circ} \mathrm{C}$ corresponded to loss of residual solvents and process aids, thermal decomposition of the base polymer, and combustion, respectively. Differential scanning calorimetry (DSC) was also conducted on simplified sporopollenin analogue 15 , which revealed no significant thermal features below $110^{\circ} \mathrm{C}$, above which data interpretation was complicated by the loss of residual solvent (Figures S58-S62). Taken together, these data demonstrate that the method for polymer crosslinking is consistent in producing sporopollenin analogues containing approximately 35 wt/wt\% DMSO which resist thermal decomposition to approximately $453{ }^{\circ} \mathrm{C}$ and do not exhibit supramolecular organization into crystalline or semi-crystalline domains. 
In addition to thermal analysis, all synthetic sporopollenin analogues disclosed were submitted to a battery of mechanical property evaluations to assess both similarity to previously disclosed properties of natural sporopollenins $(2,3)$ and potential commercial utility as structurally sound polymeric materials. Durometer hardness testing of the analogues revealed almost uniform results averaging $71 \pm 5$ Shore $D$ regardless of crosslinker length, crosslinking density, and PVA average molecular weight (Table S4). This value is higher than that of high-density polyethylene (65 Shore D) (25), commonly utilized in commercial thermoplastic hard hats. We further performed compression testing of the analogues with controlled crosslinker length, crosslinker density, and PVA average molecular weight up to a stress of approximately $200 \mathrm{MPa}$, measuring their nominal stress versus nominal strain curves, Young's moduli, and hysteresis ratios (Tables S5-S6, Figures S21-S40). Compressive moduli ranged from $97 \pm 8 \mathrm{MPa}$ to $230 \pm 36 \mathrm{MPa}$ across all analogues with a crosslinker length of $\mathrm{C} 12$ yielding the maximal observed modulus value (Figure 4A). Due to the low solvent content of the polymers, both elastically active chain density and inter-/intramolecular interactions likely contribute to the moduli of all analogues (26). Since crosslinker density is proportional to elastically active chain density but inversely proportional to inter-/intramolecular interactions, there is no strong dependence of moduli on crosslinker density observed experimentally (Figure 4B). In addition, no strong dependence on PVA average molecular weight on moduli was observed (Figure 4C), which suggests inter-/intramolecular interactions of analogues with different PVA average molecular weights are similar. It is notable that in all instances observed, synthetic analogue $\mathbf{2 6}$ demonstrated decreased modulus compared to the analogous simplified synthetic analogue 15, suggesting that the presence of coumaryl substituents suppresses inter-/intramolecular interactions of analogues, thereby leading to reduced modulus (27). The hysteresis ratio across analogues varied between $0.48 \pm 0.01$ and $0.63 \pm 0.01$, indicating significant dissipation of energy over one compressive cycle. The dissipation of energy is recoverable (Figure S42). In addition, large plasticity and rate dependency are observed across all analogues in tensile testing (Figures S41, S43). The recoverable dissipation of energy, large plasticity, and rate dependency suggests the presence of residual acetic or sulfuric acid, utilized as a catalyst for polymer crosslinking, renders the dynamic forming and reforming of crosslinks between $\alpha, \omega$-dialdehydes and PVA chains at highly deformed states. In addition to compression and tensile tests, the swelling ratio of all analogues were also measured (Tables S7-S8). As shown in Figure 4D, the weight swelling ratio decreases from 1.71 to 1.02 as crosslinker density increases from $5 \%$ to $50 \%$. Notably, a plateau in the decrease in swelling ratio with increasing crosslinker density occurs between $20 \%$ and $30 \%$ crosslinking; the same level of crosslinking previously observed in natural $P$. rigida sporopollenin 
(12). This suggests that plants might have been subject to evolutionary pressure to minimize the swelling of their sporopollenin and demonstrates that they have successfully adapted in the most efficient manner possible. Overall, these thermal and mechanical analyses reveal the unique properties of natural and unnatural sporopollenin analogues and their potential to broadly impact the field of materials science.

Plant molecular biologists have long searched for plant genes likely involved in plant sporopollenin biosynthesis (1). At present, at least 11 enzyme-encoding genes from the model plant Arabidopsis thaliana have been implicated, on the basis of single and multiple mutant phenotypes, in pollen exine development (1). These genes include fatty acid reductase MALE STERILITY 2 (MS2), ACYL-COA SYNTHETASE 5 (ACOS5), POLYKETIDE SYNTHASE A/B (PKSA/B), TETRAKETIDE a-PYRONE REDUCTASE 1/2 (TKPR1/2), strictosidine synthase-like LESS ADHESIVE POLLEN 3 (LAP3), CYP703A2, CYP704B1, and IRREGULAR POLLEN EXINE $1 / 2(I P E 1 / 2)$ (1). As the precise roles and ordering of these enzymes in the sporopollenin biosynthetic pathway remain an open field for future research, we note that our current study provides a framework for synthesizing a variety of isotopically labeled, stable pathway intermediates that can be used as chemical tools to probe various sporopollenin biosynthetic hypotheses. Such efforts may ultimately enable bioengineering of sporopollenin biosynthesis in non-reproductive tissues of plants with potential applications ranging from enhancing plant biotic and abiotic stress resistance to carbon sequestration.

In summary, we report the first synthetic recapitulation of plant sporopollenin and analogues thereof based upon that of $P$. rigida (12). The synthetic sporopollenin analogues harbor numerous favorable chemical, thermal, and mechanical properties of robust polymers with potential industrial applications. These efforts have resulted in the validation of significant portions of our previous structural model for $P$. rigida sporopollenin (12), granted chemical access to an underexplored class of bioinspired and biocompatible polymers, revealed key structure-property relationships for the engineering of sporopollenin analogues, and provided chemical tools for the future elucidation of sporopollenin biosynthesis. This work affords foundational principles which will inform the development and application of robust sporopollenin-inspired polymers in the areas of pharmaceutical encapsulation, anti-fouling agents, and chemically-inert coatings while simultaneously demystifying one of Nature's most enigmatic materials. 
Acknowledgements: This work was supported by the Grantham Foundation (J.-K.W.). We thank Dr. Walter Massefski, Dr. Bruce Adams, Dr. Mohan Kumar, and John Grimes for assistance with NMR spectroscopy and DART-TOF spectrometry, Tenzin Kunchok for assistance with mass spectrometry, and the MIT Institute for Solider Nanotechnologies for access to material characterization instrumentation.

Author Contributions: C.M.G. and J.-K.W. designed the research. C.M.G. designed and executed the synthesis of simplified and synthetic crosslinkers, their polymerization with polyvinyl alcohol, and the spectroscopic and degradative spectrometric characterization thereof. C.M.G., S.L., and J.N. mechanically characterized the polymers. All authors interpreted the results and contributed to the manuscript.

Competing Interests: The authors declare no competing interests. 


\section{Figures}

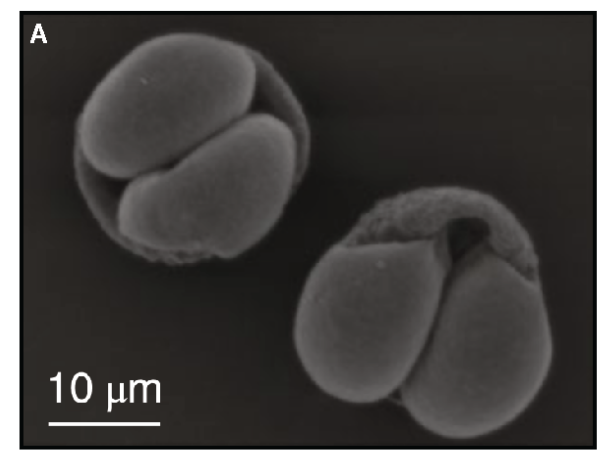

B Elucidation Workflow

$$
P \text {. rigida pollen }
$$

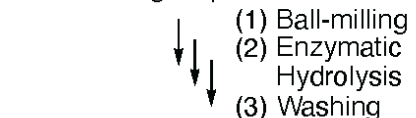

$P$ rigida sporopol

$$
\begin{aligned}
& \text { (2) Enzymatic } \\
& \text { Hydrolysis } \\
& \text { (3) Washing }
\end{aligned}
$$

Thioacidolysis

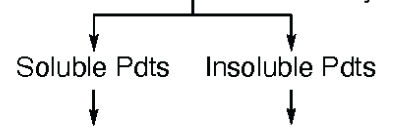

Solution State MAS SSNMR

NMR

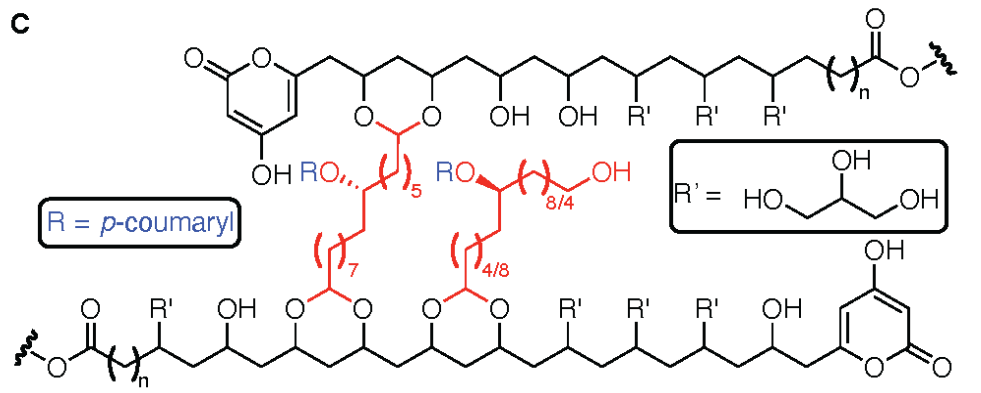

Fig. 1. Summary of the current molecular structural model of pine sporopollenin. (A) Electron micrograph of $P$. rigida pollen (12), (B) workflow employed in the structural elucidation of $P$. rigida sporopollenin, and (C) previously proposed ${ }^{13} \mathrm{C}$ MAS ssNMR averaged structure of $P$. rigida sporopollenin (structural notes: pyrone may be substituted by an ester moiety, approximately $15 \%$ of aliphatic units are singly crosslinked through one of the two alternative ends, higher dimensional crosslinking is likely) (12). 


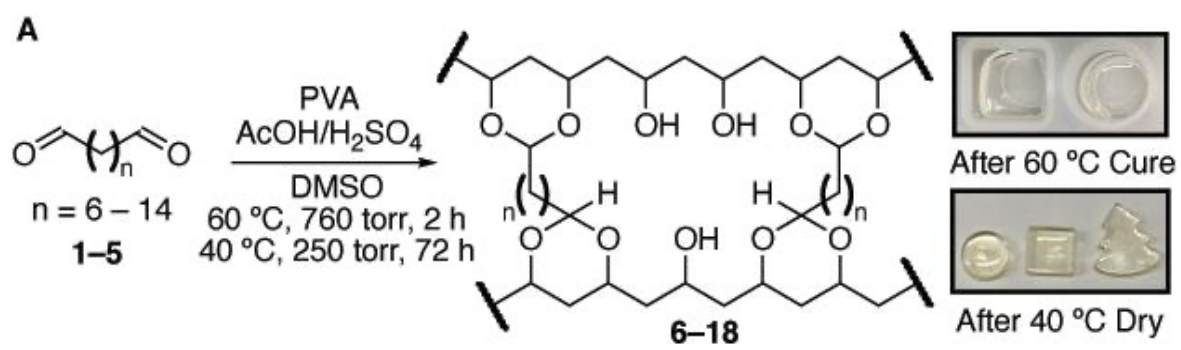<smiles>C=CC(C)Br</smiles>

19

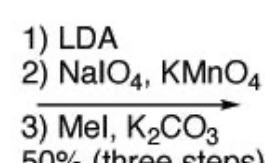
$50 \%$ (three steps)<smiles>COC(=O)C1CCCCC1=O</smiles>

21

C

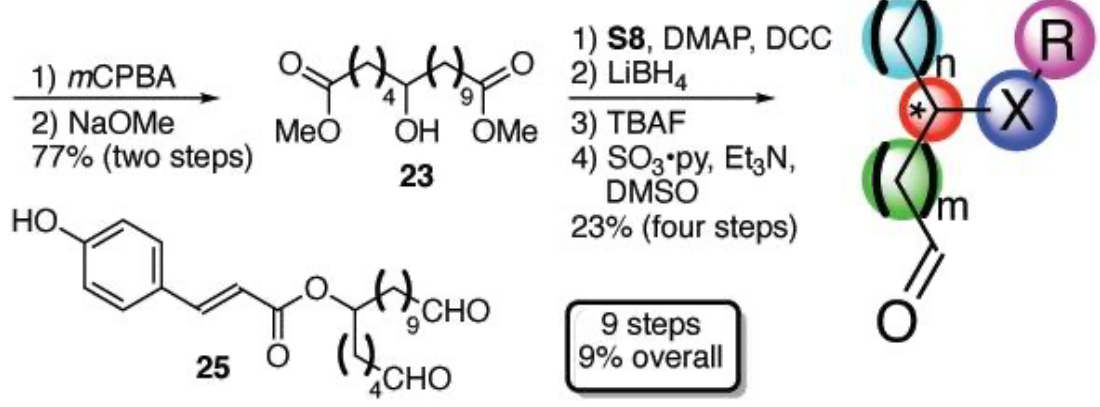

Fig. 2. Chemical synthesis of sporopollenin analogues. (A) Simplified sporopollenin analogue 618 synthesis, (B) synthetic preparation of the substituted crosslinker 25, and (C) summary of potential crosslinker analogue route divergence (teal/green: linker length modifiable via selection of cyclic ketone and bromoalkene, red: stereochemistry set via enantioselective alkylation or ring expansion, blue: heteroatom altered via selection of ring expansion conditions, purple: substituent varied via esterifications, amide couplings, alkylations, inter alia). 
A $\mathrm{HO}$

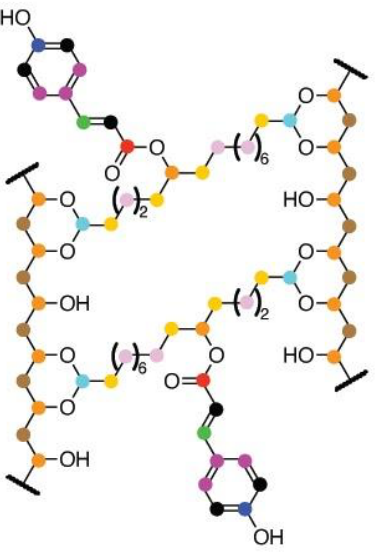

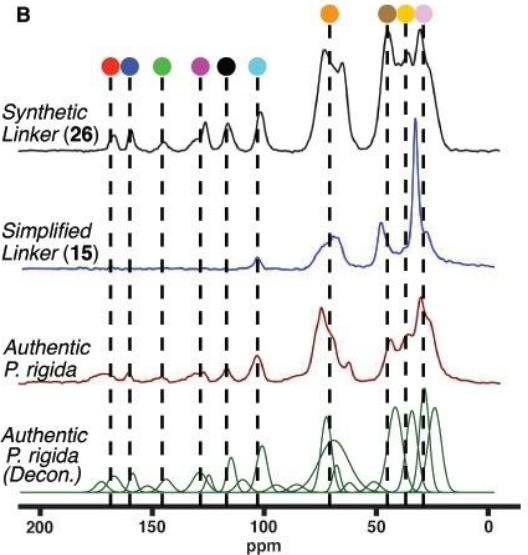

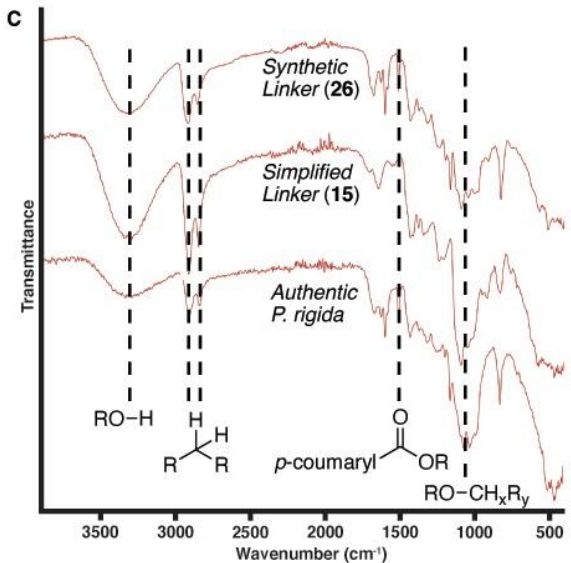



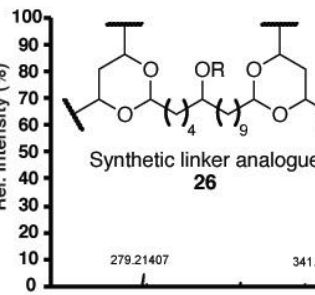

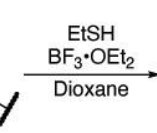

SEt

$R=0$


341.23301

398.34504

567.29935

674.37499

Fig. 3. Structural analyses of synthetic sporopollenin analogues. (A) Molecular structure of synthetic sporopollenin analogue 26, (B) ${ }^{13} \mathrm{C}$ MAS ssNMR comparison (black: synthetic linker 26 analogue, blue: simplified linker 15 analogue, red: authentic $P$. rigida sporopollenin, green: computationally deconvoluted authentic $P$. rigida sporopollenin) (12), (C) ATR-FTIR comparison (top: synthetic linker 26 analogue, middle: simplified linker 15 analogue, bottom: authentic $P$. rigida sporopollenin), and (D) DT/LC-HRMS analysis of synthetic sporopollenin 26. 
A

Stress vs. Strain

Linker Carbon Count

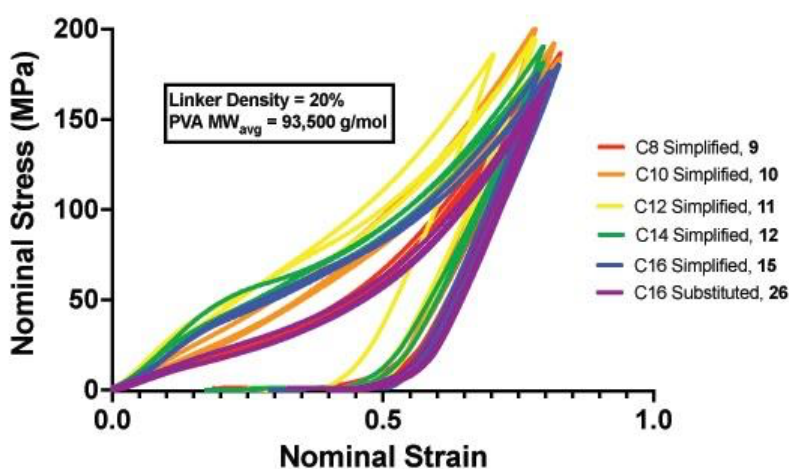

C

Stress vs. Strain

PVA Avg. MW

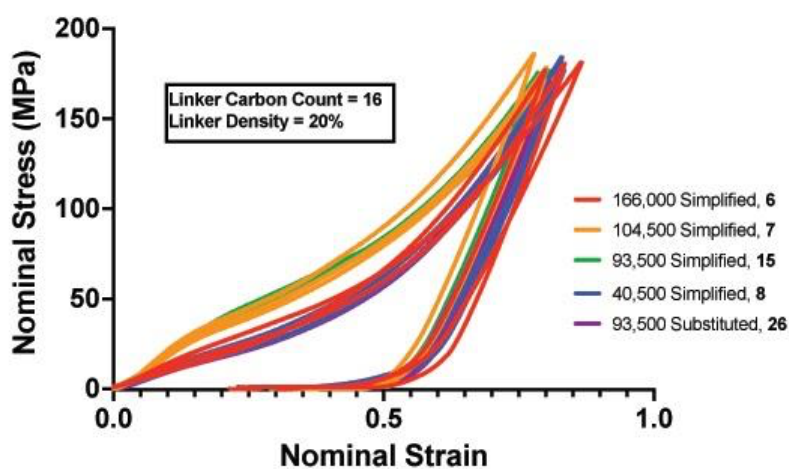

B

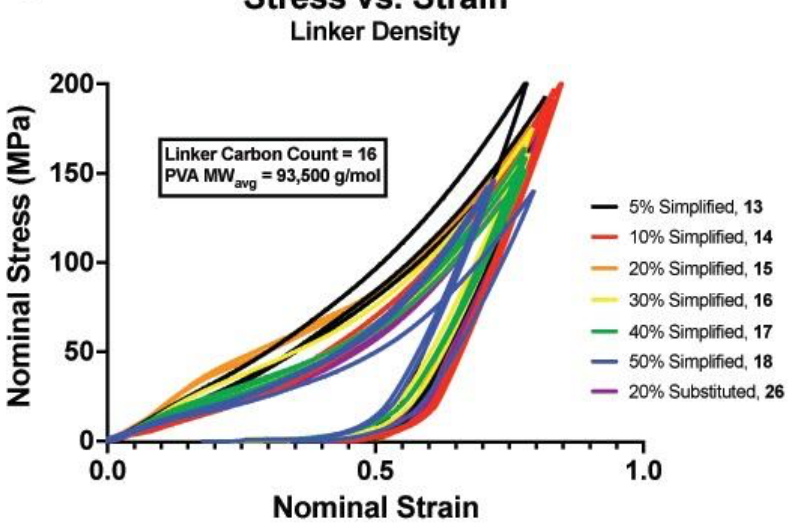

D Weight Swelling Ratio vs. Linker Density

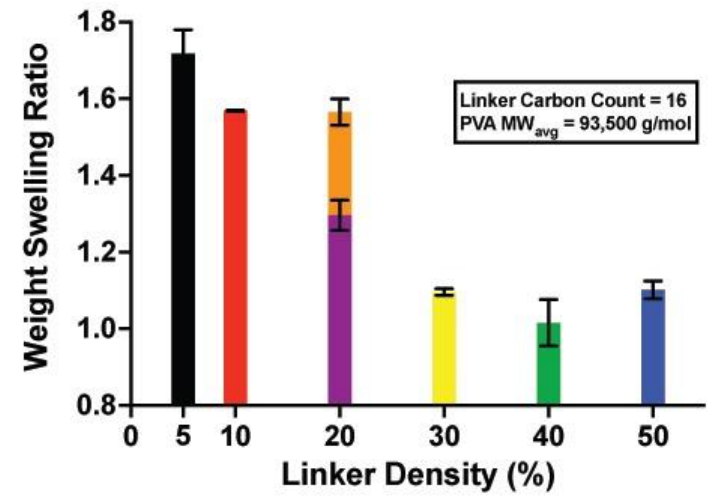

Fig. 4. Comparison of compressive stress-strain curves of synthetic sporopollenin analogues organized by (A) crosslinker length, (B) crosslinker density, and (C) PVA average molecular weight and (D) relationship between weight swelling ratio and crosslinker density. 


\section{References}

1. T. Ariizumi, K. Toriyama, Genetic Regulation of Sporopollenin Synthesis and Pollen Exine Development. Annual Review of Plant Biology. 62 (2011), pp. 437-460.

2. Z. Qu, J. C. Meredith, The atypically high modulus of pollen exine. J. R. Soc. Interface. 15 (2018), doi:10.1098/rsif.2018.0533.

3. W. Montgomery, C. Potiszil, J. S. Watson, M. A. Sephton, Sporopollenin, a Natural Copolymer, is Robust under High Hydrostatic Pressure. Macromolecular Chemistry and Physics. 217 (2016), pp. 2494-2500.

4. D. Southworth, Solubility of Pollen Exines. American Journal of Botany. 61 (1974), p. 36.

5. G. Shaw, M. Sykes, R. W. Humble, G. Mackenzie, D. Marsden, E. Pehlivan, The use of modified sporopollenin from Lycopodium clavatum as a novel ion- or ligand-exchange medium. Reactive Polymers, lon Exchangers, Sorbents. 9 (1988), pp. 211-217.

6. G. Mackenzie, G. Shaw, Sporopollenin. A novel, naturally occurring support for solid phase peptide synthesis. Int. J. Pept. Protein Res. 15, 298-300 (1980).

7. M. Sahin, I. H. Gubbuk, N. Kocak, Synthesis and Characterization of SporopolleninSupported Schiff Bases and Ruthenium(III) Sorption Studies. Journal of Inorganic and Organometallic Polymers and Materials. 22 (2012), pp. 1279-1286.

8. S. F. F. S. Yaacob, S. F. F. Yaacob, N. S. A. Razak, T. T. Aun, S. K. M. Rozi, A. K. M. Jamil, $S$. Mohamad, Synthesis and characterizations of magnetic bio-material sporopollenin for the removal of oil from aqueous environment. Industrial Crops and Products. 124 (2018), pp. 442-448.

9. S. U. Atwe, Y. Ma, H. S. Gill, Pollen grains for oral vaccination. J. Control. Release. 194, 45-52 (2014).

10. A. Diego-Taboada, L. Maillet, J. H. Banoub, M. Lorch, A. S. Rigby, A. N. Boa, S. L. Atkin, G. Mackenzie, Protein free microcapsules obtained from plant spores as a model for drug delivery: ibuprofen encapsulation, release and taste masking. J. Mater. Chem. B. 1 (2013), pp. 707-713.

11. S. A. Hamad, A. F. K. Dyab, S. D. Stoyanov, V. N. Paunov, Encapsulation of living cells into sporopollenin microcapsules. Journal of Materials Chemistry. 21 (2011), p. 18018.

12. F.-S. Li, P. Phyo, J. Jacobowitz, M. Hong, J.-K. Weng, The molecular structure of plant sporopollenin. Nat Plants. 5, 41-46 (2019).

13. A. Lutzke, K. J. Morey, J. I. Medford, M. J. Kipper, Detailed characterization of Pinus ponderosa sporopollenin by infrared spectroscopy. Phytochemistry. 170 (2020), p. 112195.

14. R. Rudra, V. Kumar, P. P. Kundu, Acid catalysed cross-linking of poly vinyl alcohol (PVA) 
by glutaraldehyde: effect of crosslink density on the characteristics of PVA membranes used in single chambered microbial fuel cells. RSC Advances. 5 (2015), pp. 83436-83447.

15. W. H. Philipp, L. C. Hsu, Three methods for in situ cross-linking of polyvinyl alcohol films for application as ion-conducting membranes in potassium hydroxide electrolyte. NASA Technical Publication. NASA-TP-1407 (1979).

16. J. Roels, P. Metz, Oxidation of a, $\omega$-Diols Using the Dess-Martin Periodinane. Synlett. 2001 (2001), pp. 0789-0790.

17. Y. Deguchi, M. Kono, Y. Koizumi, Y.-I. Izato, A. Miyake, Study on Autocatalytic Decomposition of Dimethyl Sulfoxide (DMSO). Organic Process Research \& Development. 24 (2020), pp. 1614-1620.

18. Y.-Y. Song, K. Kinami, A. Kato, Y.-M. Jia, Y.-X. Li, G. W. J. Fleet, C.-Y. Yu, First total synthesis of (+)-broussonetine W: glycosidase inhibition of natural product \& analogs. Org. Biomol. Chem. 14, 5157-5174 (2016).

19. A. Baeyer, V. Villiger, Einwirkung des Caro'schen Reagens auf Ketone. Berichte der deutschen chemischen Gesellschaft. 32 (1899), pp. 3625-3633.

20. B. Neises, W. Steglich, Simple Method for the Esterification of Carboxylic Acids. Angewandte Chemie International Edition in English. 17, 522-524 (1978).

21. S. X. Liu, H. Z. Jin, L. Shan, H. W. Zeng, B. Y. Chen, Q. Y. Sun, W. D. Zhang, Inhibitory effect of 4,4'-dihydroxy-a-truxillic acid derivatives on NO production in lipopolysaccharide-induced RAW 264.7 macrophages and exploration of structure-activity relationships. Bioorg. Med. Chem. Lett. 23, 2207-2211 (2013).

22. W. J. Guilford, D. M. Schneider, J. Labovitz, S. J. Opella, High resolution solid state C NMR spectroscopy of sporopollenins from different plant taxa. Plant Physiol. 86, 134-136 (1988).

23. B. Reif, S. E. Ashbrook, L. Emsley, M. Hong, Solid-state NMR spectroscopy. Nature Reviews Methods Primers. 1 (2021), , doi:10.1038/s43586-020-00002-1.

24. S. Mukherjee, A. Gowen, A review of recent trends in polymer characterization using nondestructive vibrational spectroscopic modalities and chemical imaging. Anal. Chim. Acta. 895, 12-34 (2015).

25. T. L. Alwardt, An Investigation of Recycled High Density Polyethylene Reinforced by Short Glass Fibers (1994).

26. M. Rubinstein, R. H. Colby, Polymer Physics (OUP Oxford, 2003).

27. R. Rutenberg, G. Golden, Y. Cohen, M. Kleiman, E. Poverenov, Investigation of Substituent Effect in Modified Nature-Sourced Polymers: Rational Side Chain Engineering to Control Yield, Design, and Properties. ACS Omega. 3, 12841-12850 (2018). 


\section{Supplementary Materials for}

Sporopollenin-inspired design and synthesis of robust materials

Christopher M. Glinkerman, Shaoting Lin, Jiahua Ni, Fu-Shuang Li, Xuanhe Zhao, Jing-Ke Weng

Correspondence to: wengj@wi.mit.edu

\section{This PDF file includes:}

General Methods

Instrument Information

Organic Synthesis

Polymer Preparation

Degree of Crosslinking (DOC) Calculation

General Method for the Preparation of Sporopollenin-Inspired Polymers

Polymer Analysis

Chemical Stability

Mechanical Analysis

Thermal Analysis

Supplementary Figures S1 to S62

References 


\section{General Methods}

All reactions were performed under nitrogen unless otherwise noted. 1,8-octanediol (TCI, >99\%), 1,10-decanediol (TCI, >95\%), 1,12-dodecanediol (Acrōs, 99\%), 1,14-tetradecanediol (CombiBlocks, 97\%), 1,16-hexadecanediol (TCI, >95\%), Dess-Martin periodinane (Oakwood, 95\%), polyvinyl alcohol $\left(\mathrm{M}_{\mathrm{w}, \mathrm{avg}}=31-50 \mathrm{k}\right.$, Sigma Aldrich, 98-99\% hydrolyzed), polyvinyl alcohol $\left(\mathrm{M}_{\mathrm{w}, \mathrm{avg}}=85-124 \mathrm{k}\right.$, Sigma Aldrich, $>99 \%$ hydrolyzed $)$, polyvinyl alcohol $\left(\mathrm{M}_{\mathrm{w}, \mathrm{avg}}=89-98 \mathrm{k}\right.$, Sigma Aldrich, $>99 \%$ hydrolyzed), polyvinyl alcohol $\left(\mathrm{M}_{\mathrm{w}, \mathrm{avg}}=146-186 \mathrm{k}\right.$, Sigma Aldrich $>99 \%$ hydrolyzed), cyclohexanone (Acrōs, >99\%), cyclohexylamine (TCI, >99\%), n-butyllithium (Acrōs, $2.45 \mathrm{M}$ ), diisopropylamine (Spectrum, >99\%), 11-bromo-1-undecene (Oakwood, 95\%), sodium (meta)periodate (BTC, 98\%), potassium permanganate (J.T. Baker, 99.4\%), methyl iodide (Alfa Aesar, $>98 \%$ ), potassium carbonate (Sigma Aldrich, 99\%), meta-chloroperbenzoic acid (Sigma Aldrich, <77\%), sodium methoxide (Acrōs, >99\%), 4-dimethylaminopyridine (Acrōs, 99\%), N,N'-dicyclohexylcarbodiimide (TCI, >98\%), lithium borohydride (Strem, 95\%), tetrabutylammonium fluoride (Oakwood, $1 \mathrm{M}$ in THF), triethylamine (Acrōs, 99\%), sulfur trioxide pyridine complex (TCI, $>95 \%$ ), coumaric acid (TCI, $>98 \%$ ), tert-butyldimethylsilyl chloride (Chem-Impex, 99\%), imidazole (Sigma Aldrich, 99\%), ethane thiol (Sigma Aldrich, 97\%), boron trifluoride diethyl etherate (Sigma Aldrich, $>46.5 \% \mathrm{BF}_{3}$ basis), acetic acid (Spectrum, $>99.7 \%$ ), concentrated sulfuric acid (EMD Millipore, 95\%), $4 \AA$ molecular sieves (Sigma Aldrich), ammonium chloride (Mallinckrodt, 99.5\%), sodium sulfate (Sigma Aldrich, >99\%), Celite (Fisher), sodium bicarbonate (Mallinckrodt, >99.7\%), conc. hydrochloric acid (Macron, 36.538.0\%), sodium hydroxide (AmericanBio, 97\%), sodium chloride (EMD Millipore, 99.5\%), dicholoromethane (Fisher, >99.5\%), dimethylsulfoxide (Fisher, $>99.9 \%$ ), diethyl ether (Fisher, $>99.9 \%$ ), tetrahydrofuran (Acrōs, $>99.5 \%$, extra dry, stabilized with BHT), acetone (Fisher, $>99.5 \%$ ), water (distilled), petroleum ether (Fisher, $36-60{ }^{\circ} \mathrm{C}$ Fraction), hexanes (Fisher, 98.5\%), ethyl acetate (Fisher, 99.5\%), dimethylformamide (Acrōs, 99.8\%), chloroform (EMD Millipore, $>99.8 \%$ ), methanol (EMD Millipore, 99.9\%), and dioxane (Acrōs, 99.8\%) were used as received without further purification unless otherwise noted. Column chromatography was conducted using Silicycle SiliaFlash $\mathrm{P} 60 \mathrm{SiO}_{2}(40-63 \mu \mathrm{m})$. Preparative TLC was conducted using Millipore $\mathrm{SiO}_{2} 60 \mathrm{~F}_{254}$ PTLC $(0.5 \mathrm{~mm})$. Analytical TLC was conducting using Millipore $\mathrm{SiO}_{2} 60$ $\mathrm{F}_{254}$ TLC $(0.250 \mathrm{~mm})$ plates. Silicone molds for polymer curing were obtained from MiniatureSweet ("Small Geometry Silicone Mold"). 


\section{Instrument Information}

Melting points were obtained using a Mel-Temp II apparatus in open capillaries and are uncorrected. Solution state ${ }^{1} \mathrm{H}$ and ${ }^{13} \mathrm{C}$ NMR spectra were obtained using either a Bruker Avance Neo $400 \mathrm{MHz}$ spectrometer equipped with a $5 \mathrm{~mm}$ BBFO SmartProbe or a Bruker Avance Neo $500 \mathrm{MHz}$ spectrometer equipped with a $5 \mathrm{~mm}$ liquid nitrogen cooled Prodigy BBO cryoprobe using either $\mathrm{CDCl}_{3}$ (Cambridge Isotope Laboratories, 99.8\%D) or DMSO- $d_{6}$ (Cambridge Isotope Laboratories, $99.9 \% \mathrm{D}$ ) as solvents. ${ }^{13} \mathrm{C}$ MAS ssNMR spectra were obtained on a Bruker Avance Neo $500 \mathrm{MHz}$ spectrometer equipped with a $3.2 \mathrm{~mm} \mathrm{HX}$ solids probe set to a MAS speed of 20 kHz. IR spectra were obtained using a Bruker Alpha 2 with a Platinum ATR accessory. High resolution mass spectrometric analysis was performed on either a JEOL AccuTOF-DART or a Thermo Fisher Scientific Q-Exactive benchtop Orbitrap. Durometer hardness testing was performed by Element Materials Technology with a Durometer Type D from Pacific Transducer Corporation. Thermogravimetric analysis was performed by Element Materials Technology with a TGA Q500 from TA Instruments. Differential scanning calorimetry was performed by Element Materials Technology with a DSC Q2000 from TA Instruments. Mechanical characterization was performed with a mechanical testing apparatus from Zwick/Roell company. 


\section{Organic Synthesis}

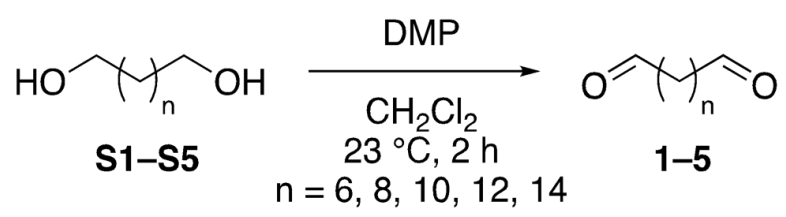

General Method for the Preparation of $\alpha, \omega$-Alkyldialdehydes (1-5). A solution of $\alpha, \omega$ alkyldiol S1-S5 $(1.00 \mathrm{mmol})$ in $\mathrm{CH}_{2} \mathrm{Cl}_{2}(10.0 \mathrm{~mL})$ at $23{ }^{\circ} \mathrm{C}$ was treated with DMP $(2.20 \mathrm{mmol})$. The resulting solution was stirred at $23{ }^{\circ} \mathrm{C}$ for $2 \mathrm{~h}$, then diluted to a total volume of $110 \mathrm{~mL}$ with $\mathrm{Et}_{2} \mathrm{O}$, washed sequentially with $1 \mathrm{~N} \mathrm{NaOH}(\mathrm{aq})(2 \times 100 \mathrm{~mL})$ and sat. $\mathrm{NH}_{4} \mathrm{Cl}(\mathrm{aq})(1 \times 100 \mathrm{~mL})$, dried over $\mathrm{Na}_{2} \mathrm{SO}_{4}$, concentrated on a rotary evaporator, and purified by flash chromatography $\left(\mathrm{SiO}_{2}, 20-50 \% \mathrm{Et}_{2} \mathrm{O} /\right.$ Hexanes $)$ to provide $\alpha, \omega$-alkyldialdehydes (1-5) as clear, colorless oils (5) or amorphous white solids (1-4) (1).

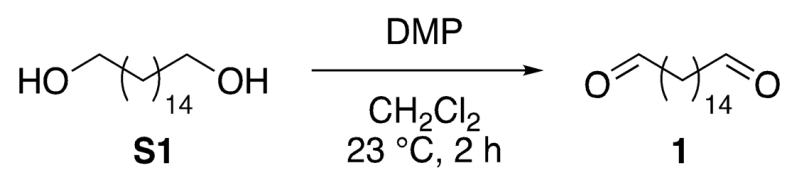

1,16-Hexadecanedial (1). Spectral data are in agreement with values published by Mangaleswaran and coworkers (2).

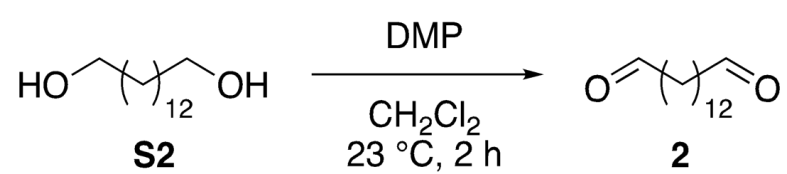

1,14-Tetradecanedial (2). Spectral data are in agreement with values published by Ziffle and coworkers (3).

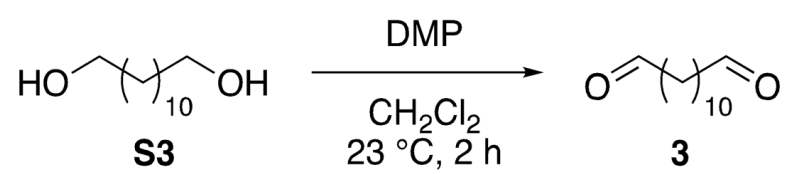

1,12-Dodecanedial (3). Spectral data are in agreement with values published by Degani and coworkers (4).

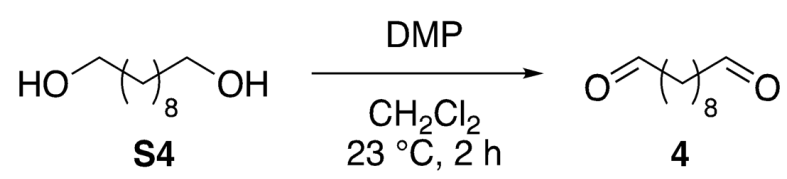

1,10-Decanedial (4). Spectral data are in agreement with values published by Guillonneau and coworkers (5).

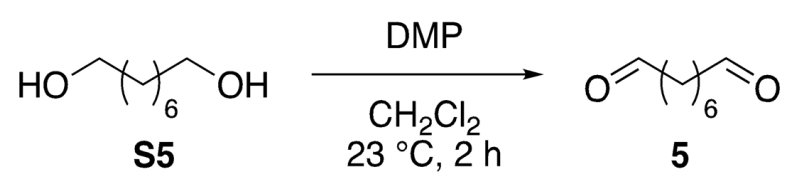

1,8-Ocatanedial (5). Spectral data are in agreement with values published by Takezawa and coworkers (6). 


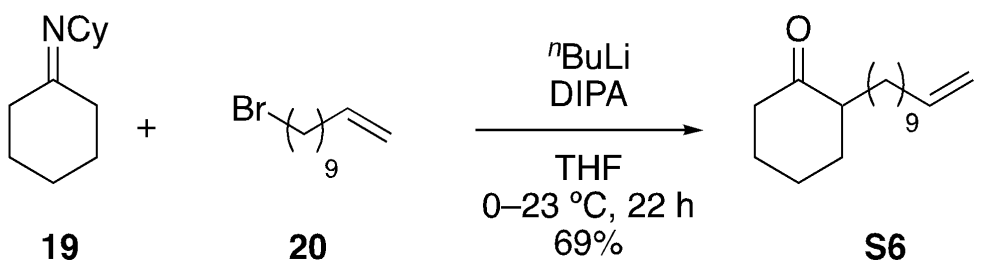

2-(Undec-10-en-1-yl)cyclohexan-1-one (S6). A solution of freshly distilled diisopropylamine $(6.77 \mathrm{~mL}, 48.0 \mathrm{mmol})$ in dry THF $(575 \mathrm{~mL})$ at $0{ }^{\circ} \mathrm{C}$ was treated dropwise with $2.45 \mathrm{M}{ }^{n} \mathrm{BuLi}$ in hexanes $(16.3 \mathrm{~mL}, 40.0 \mathrm{mmol})$ over $5 \mathrm{~min}$. The resulting solution was stirred at $0{ }^{\circ} \mathrm{C}$ for $10 \mathrm{~min}$ and then treated with a solution of freshly distilled $N$-cyclohexylcyclohexanimine (19) (7) (5.75 g, $32.1 \mathrm{mmol})$ in dry THF $(50.0 \mathrm{~mL})$. The bright yellow solution, thus obtained, was stirred at $0{ }^{\circ} \mathrm{C}$ for $1 \mathrm{~h}$ and then treated with a solution of 11-bromoundec-1-ene (20) (3.73 g, $16.0 \mathrm{mmol})$ in dry THF $(100 \mathrm{~mL})$. The reaction mixture was warmed to $23{ }^{\circ} \mathrm{C}$ and allowed to stir at $23{ }^{\circ} \mathrm{C}$ for $20 \mathrm{~h}$. After $20 \mathrm{~h}$, the resulting solution was poured into sat. $\mathrm{NH}_{4} \mathrm{Cl}(\mathrm{aq})(300 \mathrm{~mL})$ and extracted with EtOAc $(3 \times 200 \mathrm{~mL})$. The combined organic layers were washed with $1 \mathrm{~N} \mathrm{HCl}(\mathrm{aq})(1 \times 200 \mathrm{~mL})$ and sat. $\mathrm{NaCl}(\mathrm{aq})(200 \mathrm{~mL})$, dried over $\mathrm{Na}_{2} \mathrm{SO}_{4}$, concentrated on a rotary evaporator, and purified by flash chromatography $\left(\mathrm{SiO}_{2}, 5 \% \mathrm{Et}_{2} \mathrm{O} /\right.$ Pet. Ether) to provide alkene $\mathbf{S 6}$ as a pale yellow oil $(2.75 \mathrm{~g}, 69 \%):{ }^{1} \mathrm{H}$ NMR $\left(\mathrm{CDCl}_{3}, 400 \mathrm{MHz}\right) \delta 5.81$ (ddt, $\left.J=17.0,10.2,6.7 \mathrm{~Hz}, 1 \mathrm{H}\right), 4.99(\mathrm{dq}, J=$ $17.2,1.7 \mathrm{~Hz}, 1 \mathrm{H}), 4.93(\mathrm{ddt}, J=10.2,2.4,1.3 \mathrm{~Hz}, 1 \mathrm{H}), 2.38(\mathrm{dtd}, J=13.6,4.3,1.3 \mathrm{~Hz}, 1 \mathrm{H}), 2.32-$ $2.22(\mathrm{~m}, 2 \mathrm{H}), 2.13-2.07(\mathrm{~m}, 1 \mathrm{H}), 2.06-1.99(\mathrm{~m}, 3 \mathrm{H}), 1.87-1.82(\mathrm{~m}, 1 \mathrm{H}), 1.80-1.72(\mathrm{~m}, 1 \mathrm{H}), 1.71-$ $1.60(\mathrm{~m}, 2 \mathrm{H}), 1.42-1.33(\mathrm{~m}, 3 \mathrm{H}), 1.29-1.16(\mathrm{~m}, 13 \mathrm{H}) ;{ }^{13} \mathrm{C} \mathrm{NMR}\left(\mathrm{CDCl}_{3}, 100 \mathrm{MHz}\right) \delta 213.8$, 139.4, 114.2, 50.9, 42.1, 34.0, 29.9, 29.7, 29.7, 29.6, 29.6, 29.3, 29.1, 28.2, 27.3, 25.0; IR (film) $v_{\max }$ 2923, 2853, 1710, 1640, 1462, 1448, 1126, 993, $908 \mathrm{~cm}^{-1}$; HRMS (DART-TOF) $\mathrm{m} / z$ 251.2390 $\left(\mathrm{C}_{17} \mathrm{H}_{30} \mathrm{O}+\mathrm{H}^{+}\right.$requires 251.2375).<smiles>C=CCC1CCCCC1=O</smiles>

S6

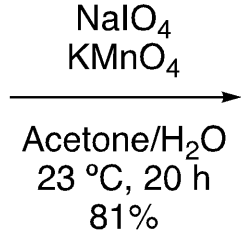

$81 \%$

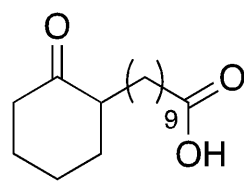

S7

10-(2-Oxocyclohexyl)decanoic acid (S7). A solution of $\mathrm{KMnO}_{4}(42.6 \mathrm{mg}, 0.270 \mathrm{mmol})$ in 1:6 acetone $/ \mathrm{H}_{2} \mathrm{O}(13.5 \mathrm{~mL})$ at $23{ }^{\circ} \mathrm{C}$ was treated with $\mathrm{NaIO}_{4}(2.31 \mathrm{~g}, 10.8 \mathrm{mmol})$, then dropwise with neat alkene S6 (135 mg, $0.539 \mathrm{mmol})$ over $5 \mathrm{~min}$. The resulting solution was stirred at $23{ }^{\circ} \mathrm{C}$ for $20 \mathrm{~h}$, then diluted to $200 \mathrm{~mL}$ total volume with $\mathrm{H}_{2} \mathrm{O}$ and extracted with EtOAc $(2 \times 100 \mathrm{~mL})$. The combined organic layers were dried over $\mathrm{Na}_{2} \mathrm{SO}_{4}$, concentrated on a rotary evaporator, and purified by flash chromatography $\left(\mathrm{SiO}_{2}, 35 \% \mathrm{Et}_{2} \mathrm{O} /\right.$ Pet. Ether) to provide carboxylic acid $\mathbf{S} 7$ as an amorphous white solid (109 mg, 81\%): mp 39-42 ${ }^{\circ} \mathrm{C}$; ${ }^{1} \mathrm{H} \mathrm{NMR}\left(\mathrm{CDCl}_{3}, 400 \mathrm{MHz}\right) \delta 10.57$ (bs, 1H), 2.41-2.22 (m, 5H), 2.12-2.00 (m, 2H), 1.87-1.59 (m, 6H), 1.43-1.15 (m, 14H); ${ }^{13} \mathrm{C} \mathrm{NMR}$ $\left(\mathrm{CDCl}_{3}, 100 \mathrm{MHz}\right) \delta 214.0,180.0,50.9,42.1,34.2,34.0,29.8,29.6,29.5,29.5,29.3,29.2,28.2$, 27.3, 24.9, 24.8; IR (film) $v_{\max } 2917,2849,1700,1429,1289,1216,952 \mathrm{~cm}^{-1}$; HRMS (DARTTOF) $m / z 269.2137\left(\mathrm{C}_{16} \mathrm{H}_{28} \mathrm{O}_{3}+\mathrm{H}^{+}\right.$requires 269.2117). 
<smiles>CC(=O)OC1CCCCC1=O</smiles>

S7

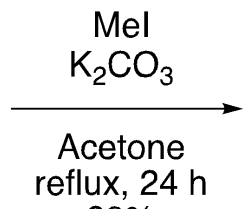

$89 \%$

Methyl 10-(2-Oxocyclohexyl)decanoate (21). A solution of carboxylic acid S7 (372 $\mathrm{mg}, 1.39$ $\mathrm{mmol})$ in acetone $(14.2 \mathrm{~mL})$ at $23{ }^{\circ} \mathrm{C}$ was treated sequentially with $\mathrm{MeI}(430 \mu \mathrm{L}, 6.93 \mathrm{mmol})$ and $\mathrm{K}_{2} \mathrm{CO}_{3}(958 \mathrm{mg}, 6.93 \mathrm{mmol})$. The resulting solution was refluxed for $24 \mathrm{~h}$, then diluted with EtOAc to a total volume of $50 \mathrm{~mL}$, washed with $1 \mathrm{~N} \mathrm{HCl}(\mathrm{aq})(1 \times 50 \mathrm{~mL})$, dried over $\mathrm{Na}_{2} \mathrm{SO}_{4}$ and concentrated on a rotary evaporator. The resulting residue was purified by flash chromatography $\left(\mathrm{SiO}_{2}, 20 \% \mathrm{Et}_{2} \mathrm{O} /\right.$ Pet. Ether) to provide methyl ester 21 as a pale yellow oil $(349 \mathrm{mg}, 89 \%):{ }^{1} \mathrm{H}$ NMR $\left(\mathrm{CDCl}_{3}, 400 \mathrm{MHz}\right) \delta 3.66(\mathrm{~s}, 3 \mathrm{H}), 2.38(\mathrm{dt}, J=14.0,4.5 \mathrm{~Hz}, 1 \mathrm{H}), 2.32-2.23(\mathrm{~m}, 4 \mathrm{H}), 2.13-$ $1.97(\mathrm{~m}, 2 \mathrm{H}), 1.87-1.57(\mathrm{~m}, 6 \mathrm{H}), 1.44-1.14(\mathrm{~m}, 14 \mathrm{H}) ;{ }^{13} \mathrm{C} \mathrm{NMR}\left(\mathrm{CDCl}_{3}, 100 \mathrm{MHz}\right) \delta 213.8$, $174.5,51.6,50.9,42.1,34.3,34.0,29.9,29.6,29.5,29.5,29.4,29.3,28.2,27.3,25.1,25.0$; IR (film) $v_{\max } 2925,2854,1737,1709,1448,1435,1196,1170 \mathrm{~cm}^{-1}$; HRMS (DART-TOF) $\mathrm{m} / z$ 283.2309 $\left(\mathrm{C}_{17} \mathrm{H}_{30} \mathrm{O}_{3}+\mathrm{H}^{+}\right.$requires 283.2273).<smiles>COC(=O)C1CCCCC1=O</smiles>

21

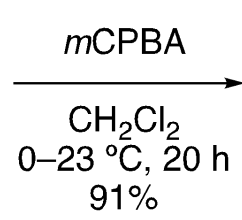

Methyl 10-(7-Oxooxepan-2-yl)decanoate (22). A solution of methyl ester 21 (78.6 mg, 0.278 $\mathrm{mmol})$ in $\mathrm{CH}_{2} \mathrm{Cl}_{2}(1.40 \mathrm{~mL})$ at $0{ }^{\circ} \mathrm{C}$ was treated with $m \mathrm{CPBA}(125 \mathrm{mg}, 0.556 \mathrm{mmol})$. The resulting solution was warmed to $23{ }^{\circ} \mathrm{C}$ and stirred at $23{ }^{\circ} \mathrm{C}$ for $20 \mathrm{~h}$. After $20 \mathrm{~h}$, the reaction mixture was diluted with $\mathrm{CH}_{2} \mathrm{Cl}_{2}$ to a total volume of $3 \mathrm{~mL}$, washed with sat. $\mathrm{NaHCO}_{3}(\mathrm{aq})(2 \times 3 \mathrm{~mL})$, dried over $\mathrm{Na}_{2} \mathrm{SO}_{4}$, concentrated on a rotary evaporator, and purified by flash chromatography $\left(\mathrm{SiO}_{2}\right.$, $\left.20-40 \% \mathrm{Et}_{2} \mathrm{O} / \mathrm{Hexanes}\right)$ to provide caprolactone 22 as an amorphous white solid (75.3 $\left.\mathrm{mg}, 91 \%\right)$ : mp 29-31 ${ }^{\circ} \mathrm{C}$; ${ }^{1} \mathrm{H} \mathrm{NMR}\left(\mathrm{CDCl}_{3}, 400 \mathrm{MHz}\right) \delta 4.21(\mathrm{dt}, J=8.2,3.9 \mathrm{~Hz}, 1 \mathrm{H}), 3.65(\mathrm{~s}, 3 \mathrm{H}), 2.62$ (qd, $J=13.7,8.7 \mathrm{~Hz}, 2 \mathrm{H}), 2.29$ (t, $J=7.6 \mathrm{~Hz}, 2 \mathrm{H}), 1.94-1.86(\mathrm{~m}, 3 \mathrm{H}), 1.74-1.41(\mathrm{~m}, 8 \mathrm{H}), 1.34-1.25$ $(\mathrm{m}, 11 \mathrm{H}) ;{ }^{13} \mathrm{C} \mathrm{NMR}\left(\mathrm{CDCl}_{3}, 100 \mathrm{MHz}\right) \delta 175.9,174,4,80.7,51.6,36.5,35.1,34.7,34.2,29.5$, 29.5, 29.4, 29.3, 29.2. 28.5, 25.5, 25.1, 23.2; IR (film) $v_{\max } 2925,2855,1726,1437,1172,1011$ $\mathrm{cm}^{-1}$; HRMS (DART-TOF) $\mathrm{m} / z$ 299.2270 $\left(\mathrm{C}_{17} \mathrm{H}_{30} \mathrm{O}_{4}+\mathrm{H}^{+}\right.$requires 299.2222).

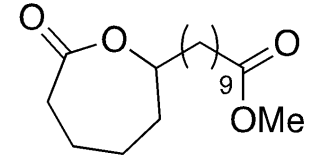

22

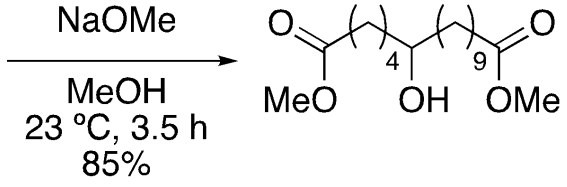

23

Dimethyl 6-Hydroxyhexadecanedioate (23). To neat caprolactone $22(1.27 \mathrm{~g}, 4.26 \mathrm{mmol})$ at $23{ }^{\circ} \mathrm{C}$ was added a $0.5 \mathrm{M}$ solution of $\mathrm{NaOMe}$ in $\mathrm{MeOH}(42.0 \mathrm{~mL}, 21.0 \mathrm{mmol})$. The resulting solution was stirred at $23{ }^{\circ} \mathrm{C}$ for $3.5 \mathrm{~h}$. After $3.5 \mathrm{~h}$, the reaction mixture was poured into sat. $\mathrm{NH}_{4} \mathrm{Cl}(\mathrm{aq})(100 \mathrm{~mL})$ and extracted with EtOAc $(3 \times 75 \mathrm{~mL})$. The combined organic layers were dried over $\mathrm{Na}_{2} \mathrm{SO}_{4}$, concentrated on a rotary evaporator, and purified by flash chromatography $\left(\mathrm{SiO}_{2}, 20-40 \%\right.$ EtOAc/Hexanes) to provide diester $\mathbf{2 3}$ as an amorphous white solid (141 $\mathrm{mg}, 85 \%$ ): 
mp 47-51 ${ }^{\circ} \mathrm{C} ;{ }^{1} \mathrm{H}$ NMR $\left(\mathrm{CDCl}_{3}, 400 \mathrm{MHz}\right) \delta 3.67(\mathrm{~s}, 3 \mathrm{H}), 3.67(\mathrm{~s}, 3 \mathrm{H}), 3.61-3.57(\mathrm{~m}, 1 \mathrm{H}), 2.31$ (dt, $J=11.2,7.5 \mathrm{~Hz}, 4 \mathrm{H}), 1.71-1.58(\mathrm{~m}, 4 \mathrm{H}), 1.50-1.35(\mathrm{~m}, 7 \mathrm{H}), 1.32-1.24(\mathrm{~m}, 12 \mathrm{H}) ;{ }^{13} \mathrm{C}$ NMR $\left(\mathrm{CDCl}_{3}, 100 \mathrm{MHz}\right) \delta 174.5,174.3,71.8,51.6,51.6,37.7,37.2,34.2,34.2,29.8,29.7,29.5,29.3$, 29.3, 25.8, 25.3, 25.1, 25.0; IR (film) $v_{\max } 3532,2911,2850,1733,1717,1246,1206,1174 \mathrm{~cm}^{-1}$; HRMS (DART-TOF) $m / z 331.2488\left(\mathrm{C}_{18} \mathrm{H}_{34} \mathrm{O}_{5}+\mathrm{H}^{+}\right.$requires 331.2484).

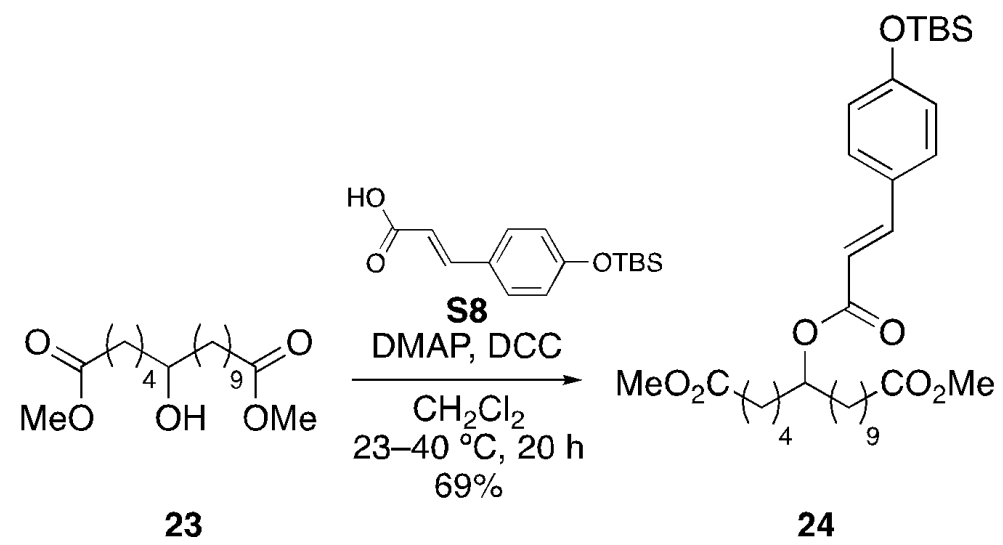

Dimethyl (E)-6-((3-(4-((tert-Butyldimethylsilyl)oxy)phenyl)acryloyl)oxy)hexadecanedioate (24). A solution of diester $23(600 \mathrm{mg}, 1.82 \mathrm{mmol})$ in dry $\mathrm{CH}_{2} \mathrm{Cl}_{2}(18.0 \mathrm{~mL})$ at $23{ }^{\circ} \mathrm{C}$ was treated sequentially with protected coumaric acid $\mathbf{S 8}(8)(811 \mathrm{mg}, 2.91 \mathrm{mmol})$, DMAP (44.4 mg, 0.363 $\mathrm{mmol})$, and DCC (563 mg, $2.73 \mathrm{mmol})$. The resulting solution was warmed to $40{ }^{\circ} \mathrm{C}$ and stirred at $40{ }^{\circ} \mathrm{C}$ for $20 \mathrm{~h}$. After $20 \mathrm{~h}$, the reaction mixture was diluted with $\mathrm{Et}_{2} \mathrm{O}(50 \mathrm{~mL})$, filtered through Celite, concentrated on a rotary evaporator, and purified by flash chromatography $\left(\mathrm{SiO}_{2}, 15-30 \%\right.$ $\mathrm{Et}_{2} \mathrm{O} /$ Hexanes) to provide coumaric ester 24 as a clear, colorless oil $(743 \mathrm{mg}, 69 \%):{ }^{1} \mathrm{H}$ NMR $\left(\mathrm{CDCl}_{3}, 500 \mathrm{MHz}\right) \delta 7.61(\mathrm{~d}, J=15.9 \mathrm{~Hz}, 1 \mathrm{H}), 7.42(\mathrm{~d}, J=8.6 \mathrm{~Hz}, 2 \mathrm{H}), 6.83(\mathrm{~d}, J=8.6 \mathrm{~Hz}, 2 \mathrm{H})$, 6.29 (d, $J=16.0 \mathrm{~Hz}, 1 \mathrm{H}), 4.99$ (qd, $J=7.2,5.2 \mathrm{~Hz}, 1 \mathrm{H}), 3.65$ (s, 3H), 3.65 (s, 3H), 2.29 (dt, $J=$ $10.5,7.6 \mathrm{~Hz}, 4 \mathrm{H}), 1.71-1.51(\mathrm{~m}, 8 \mathrm{H}), 1.43-1.24(\mathrm{~m}, 14 \mathrm{H}), 0.98(\mathrm{~s}, 9 \mathrm{H}), 0.21(\mathrm{~s}, 6 \mathrm{H}) ;{ }^{13} \mathrm{C} \mathrm{NMR}$ $\left(\mathrm{CDCl}_{3}, 125 \mathrm{MHz}\right) \delta 174.5,174.2,167.3,157.9,144.3,129.8,128.0,120.6,116.4,74.0,51.6$, 51.6, 34.4, 34.2, 34.1, 29.6, 29.6, 29.5, 29.3, 29.3, 25.8, 25.8, 25.8, 25.5, 25.1, 25.0, 18.4, -4.3; IR (film) $v_{\max } 2929,2856,1737,1706,1634,1509,1254,1164,908,836,781 \mathrm{~cm}^{-1}$; HRMS (DARTTOF) $\mathrm{m} / z 591.3795\left(\mathrm{C}_{33} \mathrm{H}_{54} \mathrm{O}_{7} \mathrm{Si}+\mathrm{H}^{+}\right.$requires 591.3717).

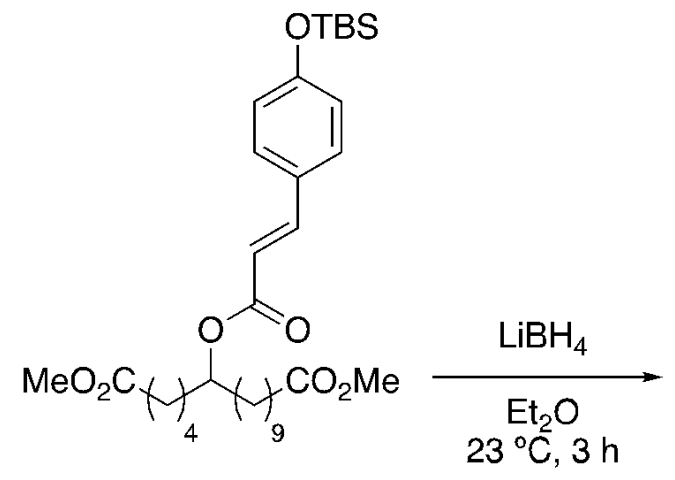

24

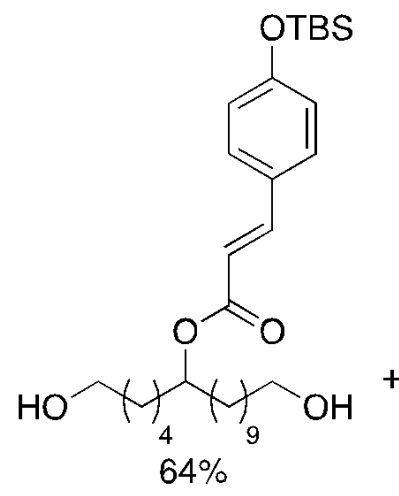

S9

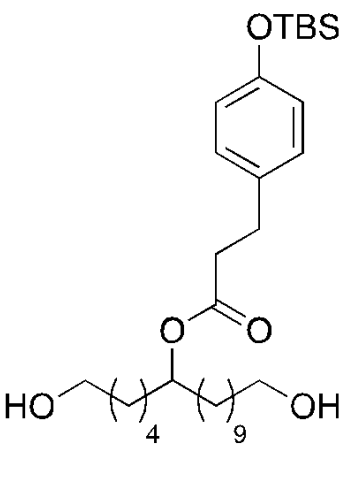

S10

1,16-Dihydroxyhexadecan-6-yl (E)-3-(4-((tert-Butyldimethylsilyl)oxy)phenyl)acrylate (S9). A solution of coumaric ester $\mathbf{2 4}(742 \mathrm{mg}, 1.26 \mathrm{mmol})$ in dry $\mathrm{Et}_{2} \mathrm{O}(15.0 \mathrm{~mL})$ at $23{ }^{\circ} \mathrm{C}$ was treated with solid $\mathrm{LiBH}_{4}(222 \mathrm{mg}, 10.0 \mathrm{mmol})$. The resulting solution was stirred at $23{ }^{\circ} \mathrm{C}$ for $3 \mathrm{~h}$. After 
$3 \mathrm{~h}$, the reaction mixture was treated dropwise with sat. $\mathrm{NaHCO}_{3}(\mathrm{aq})(15 \mathrm{~mL})$. Stirring was continued until off-gassing ceased. The biphasic mixture, thus obtained, was extracted with $\mathrm{Et}_{2} \mathrm{O}$ $(3 \times 10 \mathrm{~mL})$ and the combined organic layers were dried over $\mathrm{Na}_{2} \mathrm{SO}_{4}$, concentrated on a rotary evaporator, and purified by flash chromatography $\left(\mathrm{SiO}_{2}, 20-60 \% \mathrm{EtOAc} / \mathrm{Hexanes}\right)$ to provide a clear, colorless oil containing the product (S9, $432 \mathrm{mg}, 64 \%)$ and over-reduced byproduct (S10, $109 \mathrm{mg})$ as an inseparable mixture. For S9: ${ }^{1} \mathrm{H}$ NMR $\left(\mathrm{CDCl}_{3}, 500 \mathrm{MHz}\right) \delta 7.61(\mathrm{~d}, J=15.9 \mathrm{~Hz}$, $1 \mathrm{H}), 7.42(\mathrm{~d}, J=8.6 \mathrm{~Hz}, 2 \mathrm{H}), 6.83(\mathrm{~d}, J=8.6 \mathrm{~Hz}, 2 \mathrm{H}), 6.30(\mathrm{~d}, J=15.9 \mathrm{~Hz}, 1 \mathrm{H}), 5.01(\mathrm{qd}, J=7.4$, $5.1 \mathrm{~Hz}, 1 \mathrm{H}), 3.63(\mathrm{t}, J=6.6 \mathrm{~Hz}, 4 \mathrm{H}), 1.66-1.45(\mathrm{~m}, 8 \mathrm{H}), 1.44-1.18(\mathrm{~m}, 18 \mathrm{H}), 0.98(\mathrm{~s}, 9 \mathrm{H}), 0.21$ $(\mathrm{s}, 6 \mathrm{H}) ;{ }^{13} \mathrm{C} \mathrm{NMR}\left(\mathrm{CDCl}_{3}, 125 \mathrm{MHz}\right) \delta 173.0,167.4,157.9,144.3,129.8,120.6,116.4,74.2,63.2$, $62.9,34.5,34.4,32.9,32.8,29.7,29.6,29.6,29.6,29.5,25.9,25.8,25.8,25.7,25.5,25.2,-4.2$; HRMS (DART-TOF) $m / z 535.3895\left(\mathrm{C}_{31} \mathrm{H}_{54} \mathrm{O}_{5} \mathrm{Si}+\mathrm{H}^{+}\right.$requires 535.3819).

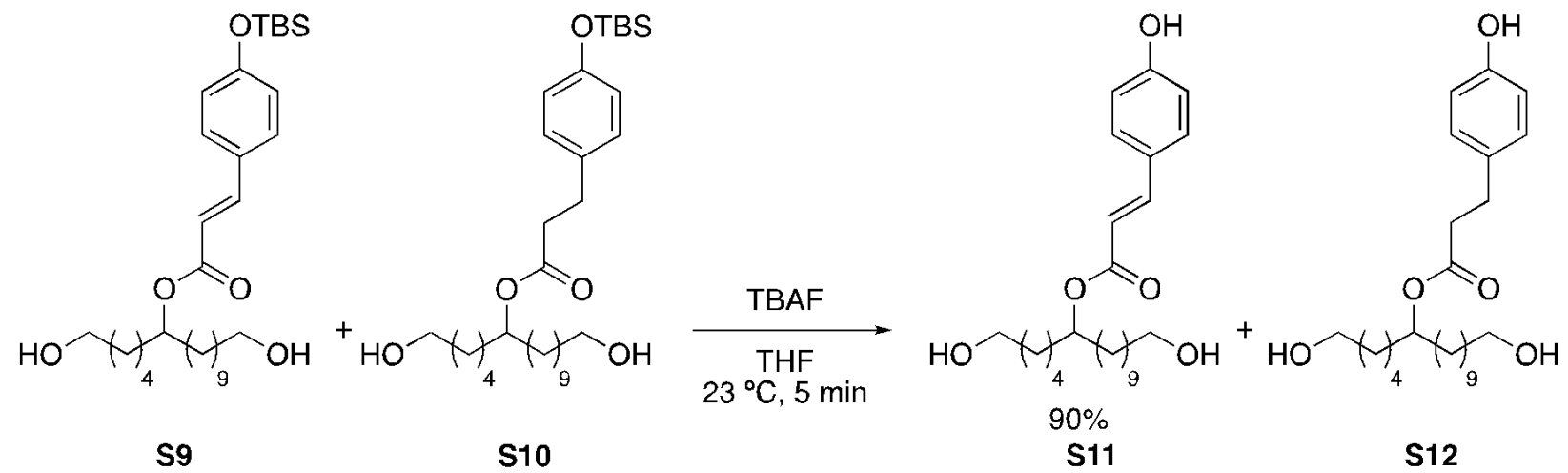

1,16-Dihydroxyhexadecan-6-yl (E)-3-(4-Hydroxyphenyl)acrylate (S11). A solution of diol S9 $(307 \mathrm{mg}, 0.574 \mathrm{mmol})$ and over-reduced byproduct $\mathbf{S 1 0}(78 \mathrm{mg})$ in THF $(13.3 \mathrm{~mL})$ at $23{ }^{\circ} \mathrm{C}$ was treated with a $1 \mathrm{M}$ solution of TBAF in THF $(1.08 \mathrm{~mL}, 1.08 \mathrm{mmol})$. The resulting solution was stirred for $5 \mathrm{~min}$. After $5 \mathrm{~min}$, the reaction mixture was treated with $1 \mathrm{M} \mathrm{HCl}(\mathrm{aq})(10 \mathrm{~mL})$ and extracted with EtOAc $(3 \times 10 \mathrm{~mL})$. The combined organic layers were dried over $\mathrm{Na}_{2} \mathrm{SO}_{4}$, concentrated on a rotary evaporator, and purified by flash chromatography $\left(\mathrm{SiO}_{2}, 80 \%\right.$ EtOAc/Hexanes) to provide a clear, colorless oil containing the triol product (S11, $217 \mathrm{mg}, 90 \%$ ) and over-reduced byproduct (S12, $55 \mathrm{mg})$ as an inseparable mixture. For $\mathbf{S 1 1}:{ }^{1} \mathrm{H}$ NMR $\left(\mathrm{CDCl}_{3}\right.$, $500 \mathrm{MHz}) \delta 7.61(\mathrm{~d}, J=15.9 \mathrm{~Hz}, 1 \mathrm{H}), 7.41(\mathrm{~d}, J=8.7 \mathrm{~Hz}, 2 \mathrm{H}), 6.84(\mathrm{~d}, J=8.6,2 \mathrm{H}), 6.47(\mathrm{bs}$, $1 \mathrm{H}), 6.28(\mathrm{~d}, J=15.9 \mathrm{~Hz}, 1 \mathrm{H}), 5.01(\mathrm{tt}, J=7.5,5.0 \mathrm{~Hz}, 1 \mathrm{H}), 3.67-3.60(\mathrm{~m}, 4 \mathrm{H}), 1.71-1.48(\mathrm{~m}$, $8 \mathrm{H}), 1.48-1.15(\mathrm{~m}, 18 \mathrm{H}) ;{ }^{13} \mathrm{C} \mathrm{NMR}\left(\mathrm{CDCl}_{3}, 125 \mathrm{MHz}\right) \delta 167.7,158.3,144.6,130.1,127.1,116.1$, 115.9, 74.3, 63.3, 62.9, 34.5, 34.4, 32.9, 32.6, 29.6, 29.6, 29.6, 29.5, 29.5, 25.8, 25.6, 25.4, 25.2; HRMS (DART-TOF) $m / z 419.2881\left(\mathrm{C}_{25} \mathrm{H}_{40} \mathrm{O}_{5}-\mathrm{H}^{+}\right.$requires 419.2798). 


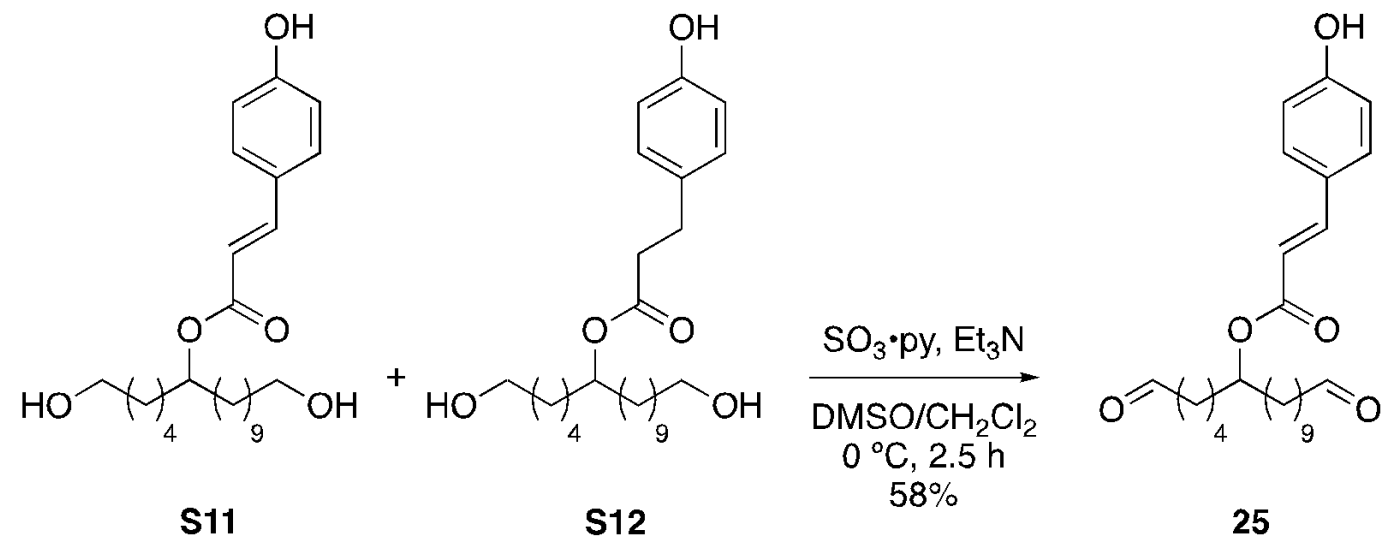

S11

S12

25

1,16-Dioxohexadecan-6-yl (E)-3-(4-Hydroxyphenyl)acrylate (25). A solution of triol S11 (94.2 $\mathrm{mg}, 0.224 \mathrm{mmol})$ and over-reduced byproduct $\mathbf{S 1 2}(23.8 \mathrm{mg})$ in $\mathrm{CH}_{2} \mathrm{Cl}_{2}(2.56 \mathrm{~mL})$ at $0{ }^{\circ} \mathrm{C}$ was treated sequentially with $\mathrm{Et}_{3} \mathrm{~N}(1.30 \mathrm{~mL})$ and a solution of $\mathrm{SO}_{3} \cdot \mathrm{py}(267 \mathrm{mg}, 1.68 \mathrm{mmol})$ in DMSO $(1.77 \mathrm{~mL})$. The resulting solution was stirred at $0{ }^{\circ} \mathrm{C}$ for $2.5 \mathrm{~h}$. After $2.5 \mathrm{~h}$, the reaction mixture was treated with $1 \mathrm{M} \mathrm{HCl}(\mathrm{aq})(5 \mathrm{~mL})$ and extracted with EtOAc $(3 \times 5 \mathrm{~mL})$. The combined organic layers were dried over $\mathrm{Na}_{2} \mathrm{SO}_{4}$, concentrated on a rotary evaporator, and purified by PTLC $\left(\mathrm{SiO}_{2}\right.$, $50 \% \mathrm{EtOAc/Hexanes)}$ to provide dialdehyde 25 as a clear, colorless oil $(54.2 \mathrm{mg}, 58 \%):{ }^{1} \mathrm{H}$ NMR (DMSO- $\left.d_{6}, 500 \mathrm{MHz}\right) \delta 10.00(\mathrm{~s}, 1 \mathrm{H}), 9.65(\mathrm{dt}, J=3.2,1.6 \mathrm{~Hz}, 2 \mathrm{H}), 7.57-7.52(\mathrm{~m}, 3 \mathrm{H}), 6.79(\mathrm{~d}$, $J=8.7 \mathrm{~Hz}, 2 \mathrm{H}), 6.38(\mathrm{~d}, J=16.0 \mathrm{~Hz}, 1 \mathrm{H}), 4.93-4.88(\mathrm{~m}, 1 \mathrm{H}), 2.43(\mathrm{td}, J=7.2,1.6 \mathrm{~Hz}, 2 \mathrm{H}), 2.39$ $(\mathrm{td}, J=7.3,1.7 \mathrm{~Hz}, 2 \mathrm{H}), 1.58-1.47(\mathrm{~m}, 8 \mathrm{H}), 1.33-1.24(\mathrm{~m}, 14 \mathrm{H}) ;{ }^{13} \mathrm{C} \mathrm{NMR}\left(\mathrm{CDCl}_{3}, 125 \mathrm{MHz}\right) \delta$ 203.4, 202.9, 167.5, 158.0, 144.5, 130.1, 127.3, 116.0, 116.0, 74.1, 44.0, 43.9, 34.4, 34.2, 29.6, 29.6, 29.4, 29.4, 29.3, 25.5, 25.1, 22.2, 22.1; IR (film) $v_{\max } 3347,2926,2854,1704,1603,1585$, 1514, 1261, 1164, 983, $832 \mathrm{~cm}^{-1}$; HRMS (DART-TOF) $m / z 417.2740\left(\mathrm{C}_{25} \mathrm{H}_{36} \mathrm{O}_{5}+\mathrm{H}^{+}\right.$requires 417.2641). 


\section{Polymer Preparation}

Degree of Crosslinking (DOC) Calculation

Formula:

$$
\frac{\text { mass } P V A}{1} \times \frac{m o l P V A}{44 g P V A} \times \frac{m o l-O H}{m o l P V A} \times \frac{\text { mol crosslinker }}{4 m o l-O H} \times \frac{g}{m o} \quad \begin{gathered}
\text { g crosslinker to achieve } \\
100 \% \text { theoretical DOC }
\end{gathered}
$$

\section{Sample Calculation:}

If $1 \mathrm{~mL}$ of $5 \mathrm{wt} \%$ PVA in DMSO is to be $20 \%$ crosslinked by dialdehyde 15 :

$$
0.05 g P V A \times \frac{\text { mol PVA }}{44 g} \times \frac{\text { mol crosslinker }}{4 \text { mol }-O H} \times \frac{254.41 \mathrm{~g} \text { crosslinker }}{\text { mol crosslinker }} \times 0.2=14.5 \mathrm{mg} \text { crosslinker }
$$

Table S1. DOC results for $50 \mathrm{mg}$ PVA crosslinked by dialdehyde 15

\begin{tabular}{|l|l|}
\hline Theoretical DOC (\%) & Mass of Linker (mg) \\
\hline 0 & 0.000 \\
\hline 1 & 0.723 \\
\hline 2 & 1.45 \\
\hline 5 & 3.63 \\
\hline 10 & 7.25 \\
\hline 20 & 14.5 \\
\hline 30 & 21.7 \\
\hline 40 & 28.9 \\
\hline 50 & 36.1 \\
\hline
\end{tabular}


General Method for the Preparation of Sporopollenin-Inspired Polymers

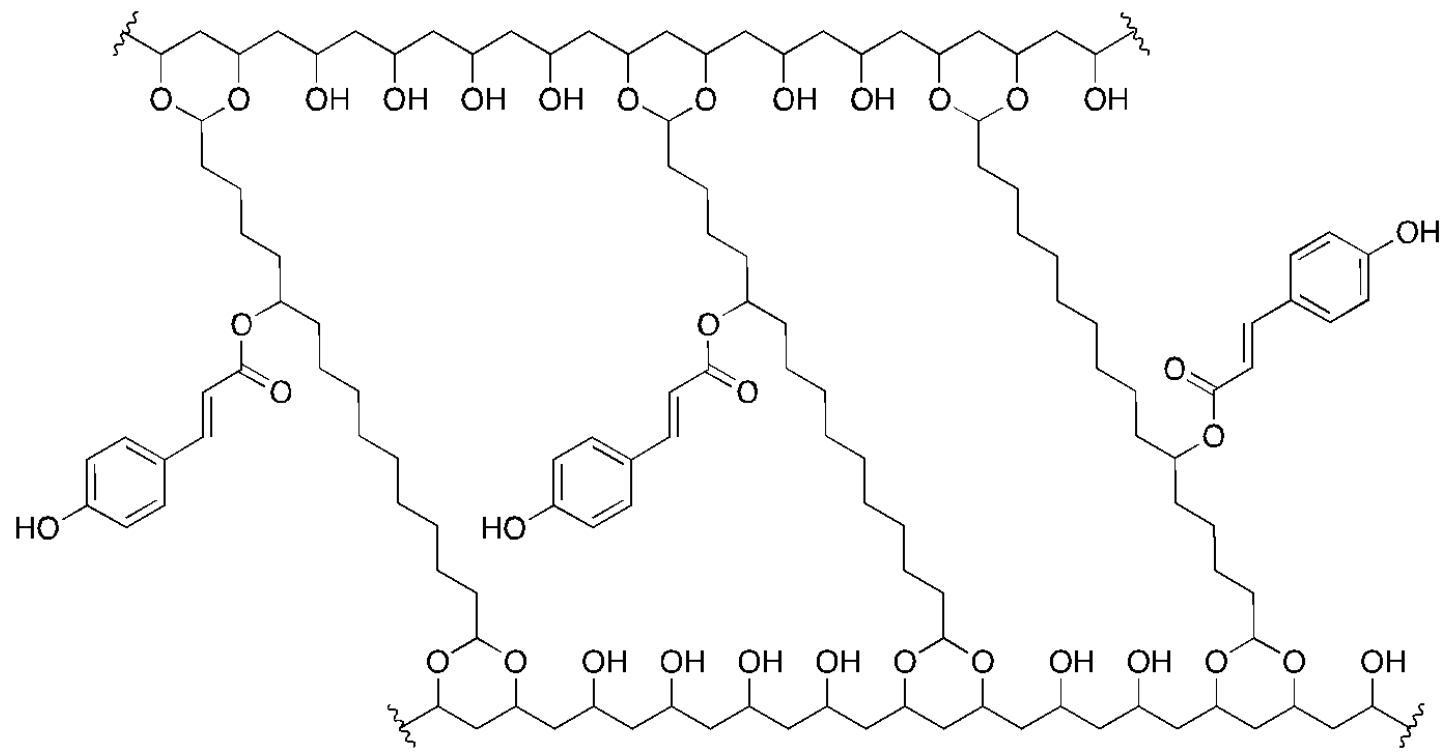

Synthetic Sporopollenin Analogues. To a solution of 5.00 wt\% PVA in DMSO (1.00 mL, 1.14 mmol) was added an appropriate amount of dialdehyde (vide supra) to achieve the desired DOC at $23{ }^{\circ} \mathrm{C}$. The suspension was heated to $60{ }^{\circ} \mathrm{C}$ in a sealed vial until complete dissolution of the dialdehyde was achieved (approx. $5 \mathrm{~min}$ ). To this warm solution was added $0.10 \mathrm{~mL}$ of acid catalyst solution containing $7.50 \mathrm{v} / \mathrm{v} \% \mathrm{AcOH}$ and $2.50 \mathrm{v} / \mathrm{v} \% \mathrm{H}_{2} \mathrm{SO}_{4}$ in DMSO. The resulting solution was vigorously homogenized and poured into silicone molds. Curing of this solution at 55-60 ${ }^{\circ} \mathrm{C}$ at 760 torr for $90-120$ min under an atmosphere of air, then at $40{ }^{\circ} \mathrm{C}$ at 250 torr for 72 $\mathrm{h}$ under an atmosphere of air provided crosslinked polymers which were subsequently washed by submerging sequentially in distilled $\mathrm{H}_{2} \mathrm{O}(10 \mathrm{~min})$, sat. $\mathrm{NaHCO}_{3}$ (aq) (10 min), and distilled $\mathrm{H}_{2} \mathrm{O}$ again $(2 \mathrm{~h})$. Drying of the resultant materials under ambient conditions for $24 \mathrm{~h}$ provided sporopollenin-inspired polymers which were utilized, without further treatment, for chemical, physical, and thermal analyses.

Table S2. Synthetic Sporopollenin Analogue Numbering Key

\begin{tabular}{|l|l|l|l|l|l|l|l|}
\hline Sporopollenin Analogue \# & $\mathbf{6}$ & $\mathbf{7}$ & $\mathbf{8}$ & $\mathbf{9}$ & $\mathbf{1 0}$ & $\mathbf{1 1}$ & $\mathbf{1 2}$ \\
\hline Linker Carbon Count (\#C) & 16 & 16 & 16 & 8 & 10 & 12 & 14 \\
\hline Linker Density (\%) & 20 & 20 & 20 & 20 & 20 & 20 & 20 \\
\hline PVA MW avg (g/mol) & 166000 & 104500 & 40500 & 93500 & 93500 & 93500 & 93500 \\
\hline Sporopollenin Analogue \# & $\mathbf{1 3}$ & $\mathbf{1 4}$ & $\mathbf{1 5}$ & $\mathbf{1 6}$ & $\mathbf{1 7}$ & $\mathbf{1 8}$ & $\mathbf{2 6}$ \\
\hline Linker Carbon Count (\#C) & 16 & 16 & 16 & 16 & 16 & 16 & $16^{*}$ \\
\hline Linker Density (\%) & 5 & 10 & 20 & 30 & 40 & 50 & 20 \\
\hline PVA MW avg (g/mol) & 93500 & 93500 & 93500 & 93500 & 93500 & 93500 & 93500 \\
\hline
\end{tabular}

* Indicates synthetic substituted crosslinker 


\section{Polymer Analysis}

\section{Chemical Stability}

\section{$\underline{\text { Solvolysis Study }}$}

\section{General Method:}

One disk of sporopollenin-inspired polymer (vide supra) of known mass was placed in $10 \mathrm{~mL}$ of solvent and allowed to shake $(150 \mathrm{rpm})$ at $23{ }^{\circ} \mathrm{C}$ for $24 \mathrm{~h}$. The polymer disk was then dried at $40^{\circ} \mathrm{C}$ and 250 torr for $24 \mathrm{~h}$ unless otherwise noted, allowed to equilibrate under ambient conditions for $24 \mathrm{~h}$, then weighed to determine total mass loss during solvolysis. All observed mass losses, with the exception of the conc. $\mathrm{H}_{2} \mathrm{SO}_{4}$ sample, are below the mass fraction attributed to volatile low molecular weight compounds (i.e. residual solvent) in the starting samples as observed by TGA (vide infra).

Table S3. Chemical stability of synthetic sporopollenin polymer 15

\begin{tabular}{|l|l|l|l|l|}
\hline Solvent & $\begin{array}{l}\text { Initial Mass } \\
(\mathrm{mg})\end{array}$ & $\begin{array}{l}\text { Final Mass } \\
(\mathrm{mg})\end{array}$ & $\begin{array}{l}\text { Mass Loss } \\
(\%)\end{array}$ & Notes: \\
\hline $\mathrm{CH}_{2} \mathrm{Cl}_{2}$ & 29.28 & 26.84 & 8.334 & N/A \\
\hline Hexanes & 28.75 & 26.86 & 6.574 & N/A \\
\hline $\begin{array}{l}\text { Ethyl } \\
\text { Acetate }\end{array}$ & 27.34 & 25.63 & 6.255 & N/A \\
\hline Acetone & 27.51 & 26.08 & 5.198 & N/A \\
\hline DMF & 27.61 & 30.00 & -8.656 & 48 h dry time \\
\hline $\mathrm{DMSO}^{\circ}$ & 27.10 & 32.02 & -18.155 & $48 \mathrm{~h}$ dry time \\
\hline $\mathrm{H}_{2} \mathrm{O}$ & 24.81 & 22.57 & 9.029 & N/A \\
\hline $\mathrm{THF}^{\mathrm{O}}$ & 26.46 & 25.13 & 5.026 & N/A \\
\hline $\mathrm{CHCl}_{3}$ & 27.27 & 25.84 & 5.140 & N/A \\
\hline $\begin{array}{l}\text { conc. } \\
\mathrm{H}_{2} \mathrm{SO}\end{array}$ & 27.11 & N/A & N/A & Blackened and crumbling \\
\hline $5 \mathrm{~N} \mathrm{NaOH}_{4}$ & 27.39 & 29.03 & -5.988 & $\begin{array}{l}48 \text { h dry time, add'l mass likely due to } \\
\text { retained NaOH in sample }\end{array}$ \\
\hline
\end{tabular}




\section{Degradation Study}

General Method:

A suspension of authentic sporopollenin or synthetic sporopollenin analogue (4.0 mg) in dry 1,4dioxane $(9.0 \mathrm{~mL})$ was treated sequentially with ethane thiol $(1.0 \mathrm{~mL}, 14 \mathrm{mmol})$ and $>46.5 \%$ boron trifluoride diethyl etherate $(0.25 \mathrm{~mL}, 0.05 \mathrm{mmol})$ at $23{ }^{\circ} \mathrm{C}$. The resulting suspension was sealed and heated to $100{ }^{\circ} \mathrm{C}$ for $4 \mathrm{~h}$. After $4 \mathrm{~h}$, the reaction mixture was cooled to $23{ }^{\circ} \mathrm{C}$, treated dropwise with sat. $\mathrm{NaHCO}_{3}(\mathrm{aq})$ to achieve a $\mathrm{pH}$ of approximately 4, and extracted with $\mathrm{CHCl}_{3}(3 \times 5 \mathrm{~mL})$. The combined organic layers were dried over $\mathrm{Na}_{2} \mathrm{SO}_{4}$ and concentrated under a stream of nitrogen. The crude product was dissolved in $\mathrm{CHCl}_{3}$, filtered, and submitted for LC-HRMS analysis (9). 


\section{Mechanical Analysis}

\section{Durometer Hardness}

General Method:

Durometer hardness measurements were conducted by Element Materials Technology in accordance with ASTM D2240 (excepting sample geometry) with conditioning at laboratory conditions of $23 \pm 2{ }^{\circ} \mathrm{C}$ and $50 \pm 10 \%$ relative humidity. Samples were disks approximately 1.4 $\mathrm{mm}$ in thickness and $3.5 \mathrm{~mm}$ in diameter. Due to the small sample size, one reading was taken from each individual sample. Hardness reported on the Shore D scale.

Table S4. Durometer hardness (Shore D) of synthetic sporopollenin analogues

\begin{tabular}{|l|l|l|l|l|l|l|l|}
\hline Sporopollenin Analogue \# & $\mathbf{6}$ & $\mathbf{7}$ & $\mathbf{8}$ & $\mathbf{9}$ & $\mathbf{1 0}$ & $\mathbf{1 1}$ & $\mathbf{1 2}$ \\
\hline Sample 1 (Shore D) & 67 & 76 & 67 & 70 & 69 & 85 & 77 \\
\hline Sample 2 (Shore D) & 69 & 77 & 69 & 68 & 69 & 84 & 75 \\
\hline Sample 3 (Shore D) & 69 & 78 & 68 & 66 & 69 & 83 & 75 \\
\hline Sample 4 (Shore D) & 70 & 77 & --- & 62 & 71 & 84 & --- \\
\hline Average (Shore D) & $\mathbf{6 9}$ & $\mathbf{7 7}$ & $\mathbf{6 8}$ & $\mathbf{6 7}$ & $\mathbf{7 0}$ & $\mathbf{8 4}$ & $\mathbf{7 6}$ \\
\hline SD (Shore D) & $\mathbf{1}$ & $\mathbf{1}$ & $\mathbf{1}$ & $\mathbf{3}$ & $\mathbf{1}$ & $\mathbf{1}$ & $\mathbf{1}$ \\
\hline & & & & & & \\
\hline Sporopollenin Analogue \# & $\mathbf{1 3}$ & $\mathbf{1 4}$ & $\mathbf{1 5}$ & $\mathbf{1 6}$ & $\mathbf{1 7}$ & $\mathbf{1 8}$ & $\mathbf{2 6}$ \\
\hline Sample 1 (Shore D) & 77 & 68 & 67 & 71 & 65 & 66 & 70 \\
\hline Sample 2 (Shore D) & 77 & 68 & 69 & 73 & 70 & 66 & 69 \\
\hline Sample 3 (Shore D) & 73 & 67 & 70 & 72 & 65 & 65 & 75 \\
\hline Sample 4 (Shore D) & --- & --- & 68 & 73 & 70 & 63 & 70 \\
\hline Average (Shore D) & $\mathbf{7 6}$ & $\mathbf{6 8}$ & $\mathbf{6 9}$ & $\mathbf{7 2}$ & $\mathbf{6 8}$ & $\mathbf{6 5}$ & $\mathbf{7 1}$ \\
\hline SD (Shore D) & $\mathbf{2}$ & $\mathbf{1}$ & $\mathbf{1}$ & $\mathbf{1}$ & $\mathbf{3}$ & $\mathbf{1}$ & $\mathbf{3}$ \\
\hline
\end{tabular}

\section{Overall:}

Average (Shore D): 71

SD (Shore D): 5 


\section{Compression Testing}

\section{General Method:}

Compression testing was conducted in triplicate according to the following procedure: We fabricated the testing samples in a disk shape with a diameter $D$ of around $3.7 \mathrm{~mm}$ and a thickness $H$ of around $1.6 \mathrm{~mm}$. The disk-shaped sample was compressed using a mechanical tester from Zwick/Roell company up to its nominal strain of $80 \%$ and subsequently unloaded to its original state. The loading speed was set as $1 \mathrm{~mm} / \mathrm{min}$. The measured nominal stress $s$ versus nominal strain $\varepsilon$ of the sample can be calculated via $s=F / A$ and $\varepsilon=\Delta / H$, where $F$ is the measured force, $A=\pi D^{2} / 4$ is the cross-sectional area of the sample with $D$ as the diameter of the sample, $\Delta$ is the loading displacement, and $H$ is the thickness of the sample. The elastic modulus of the sample was calculated via $E=d s /\left.d \varepsilon\right|_{\varepsilon=0}$. The hysteresis ratio was calculated by $h=\int_{0}^{\varepsilon_{\max }} s d \varepsilon$, where $\varepsilon_{\text {max }}$ is the maximum nominal strain during the cyclic compressive loading.

Table S5. Moduli (MPa) of synthetic sporopollenin analogues

\begin{tabular}{|c|c|c|c|c|c|c|c|}
\hline Sporopollenin Analogue \# & 6 & 7 & 8 & 9 & 10 & 11 & 12 \\
\hline Sample $1(\mathrm{MPa})$ & 120 & 143 & 112 & 95 & 115 & 200 & 210 \\
\hline Sample $2(\mathrm{MPa})$ & 100 & 184 & 103 & 90 & 136 & 270 & 188 \\
\hline Sample $3(\mathrm{MPa})$ & 134 & 170 & 92 & 105 & 148 & 220 & 192 \\
\hline Average & 118 & 166 & 102 & 97 & 133 & 230 & 197 \\
\hline SD & 17 & 21 & 10 & 8 & 17 & 36 & 12 \\
\hline Sporopollenin Analogue \# & 13 & 14 & 15 & 16 & 17 & 18 & 26 \\
\hline Sample $1(\mathrm{MPa})$ & 158 & 105 & 156 & 157 & 120 & 86 & 90 \\
\hline Sample $2(\mathrm{MPa})$ & 111 & 107 & 160 & 135 & 106 & 100 & 86 \\
\hline Sample $3(\mathrm{MPa})$ & 131 & 87 & 175 & 116 & 118 & 95 & 116 \\
\hline Average & 133 & 100 & 164 & 136 & 115 & 94 & 97 \\
\hline SD & 24 & 11 & 10 & 21 & 8 & 7 & 16 \\
\hline
\end{tabular}

\section{Overall:}

Average (MPa): 134

SD (MPa): 41 
Table S6. Hysteresis ratio (dimensionless) of synthetic sporopollenin analogues

\begin{tabular}{|l|l|l|l|l|l|l|l|}
\hline Sporopollenin Analogue \# & $\mathbf{6}$ & $\mathbf{7}$ & $\mathbf{8}$ & $\mathbf{9}$ & $\mathbf{1 0}$ & $\mathbf{1 1}$ & $\mathbf{1 2}$ \\
\hline Sample 1 & 0.54 & 0.62 & 0.53 & 0.48 & 0.53 & 0.62 & 0.58 \\
\hline Sample 2 & 0.54 & 0.62 & 0.55 & 0.49 & 0.53 & 0.60 & 0.60 \\
\hline Sample 3 & 0.59 & 0.63 & 0.54 & 0.47 & 0.52 & 0.59 & 0.59 \\
\hline Average & $\mathbf{0 . 5 6}$ & $\mathbf{0 . 6 3}$ & $\mathbf{0 . 5 4}$ & $\mathbf{0 . 4 8}$ & $\mathbf{0 . 5 2}$ & $\mathbf{0 . 6 0}$ & $\mathbf{0 . 5 9}$ \\
\hline SD & $\mathbf{0 . 0 3}$ & $\mathbf{0 . 0 1}$ & $\mathbf{0 . 0 1}$ & $\mathbf{0 . 0 1}$ & $\mathbf{0 . 0 1}$ & $\mathbf{0 . 0 1}$ & $\mathbf{0 . 0 1}$ \\
\hline & & & & & & & \\
\hline Sporopollenin Analogue \# & $\mathbf{1 3}$ & $\mathbf{1 4}$ & $\mathbf{1 5}$ & $\mathbf{1 6}$ & $\mathbf{1 7}$ & $\mathbf{1 8}$ & $\mathbf{2 6}$ \\
\hline Sample 1 & 0.58 & 0.56 & 0.63 & 0.55 & 0.55 & 0.55 & 0.57 \\
\hline Sample 2 & 0.58 & 0.57 & 0.63 & 0.55 & 0.54 & 0.56 & 0.56 \\
\hline Sample 3 & 0.60 & 0.55 & 0.64 & 0.57 & 0.55 & 0.56 & 0.58 \\
\hline Average & $\mathbf{0 . 5 9}$ & $\mathbf{0 . 5 6}$ & $\mathbf{0 . 6 3}$ & $\mathbf{0 . 5 6}$ & $\mathbf{0 . 5 5}$ & $\mathbf{0 . 5 6}$ & $\mathbf{0 . 5 7}$ \\
\hline SD & $\mathbf{0 . 0 1}$ & $\mathbf{0 . 0 1}$ & $\mathbf{0 . 0 1}$ & $\mathbf{0 . 0 1}$ & $\mathbf{0 . 0 1}$ & $\mathbf{0 . 0 1}$ & $\mathbf{0 . 0 1}$ \\
\hline
\end{tabular}

\section{Overall:}

Average (dimensionless): 0.53

SD (dimensionless): 0.15 


\section{Tensile Testing}

General Method:

Tensile testing was conducted in triplicate according to the following procedure: We fabricated the testing samples in a strip shape with a width $W$ of around $8.6 \mathrm{~mm}$, a thickness $T$ of around 1.6 $\mathrm{mm}$, and a height $H$ of around $10 \mathrm{~mm}$. A monotonic tensile load was applied on the strip-shaped sample using a mechanical tester from Zwick/Roell company up to the rupture of the sample. The loading speed was set as $1 \mathrm{~mm} / \mathrm{min}$. The measured nominal stress $s$ versus nominal strain $\varepsilon$ of the sample can be calculated via $s=F / A$ and $\varepsilon=\Delta / H$, where $F$ is the measured force, $A=W T$ is the cross-sectional area of the sample with $W$ as the width of the sample and $T$ as the thickness of the sample, $\Delta$ is the loading displacement, and $H$ is the height of the sample. We also performed a cyclic tensile loading on the sample at the same loading speed of $1 \mathrm{~mm} / \mathrm{min}$, measuring its hysteresis at various applied strains under tensile loading. 


\section{Swelling Ratio}

General Method:

The measurement of swelling ratio was conducted in triplicate according to the following procedure: We fabricated the testing samples in a disc shape with a diameter $D$ of around $3.7 \mathrm{~mm}$ and a thickness $H$ of around $1.6 \mathrm{~mm}$. Both the volume and weight of the sample were measured before and after immersing in a deionized water, measuring the volume swelling ratios and the weight swelling ratios, respectively.

Table S7. Volume swelling ratios (dimensionless) of synthetic sporopollenin analogues

\begin{tabular}{|l|l|l|l|l|l|l|l|}
\hline Sporopollenin Analogue \# & $\mathbf{6}$ & $\mathbf{7}$ & $\mathbf{8}$ & $\mathbf{9}$ & $\mathbf{1 0}$ & $\mathbf{1 1}$ & $\mathbf{1 2}$ \\
\hline Sample 1 & 1.24 & 1.47 & 1.38 & 1.51 & 1.44 & 1.62 & 1.53 \\
\hline Sample 2 & 1.28 & 1.41 & 1.35 & 1.56 & 1.40 & 1.57 & 1.50 \\
\hline Sample 3 & 1.27 & 1.39 & 1.39 & 1.54 & 1.41 & 1.70 & 1.51 \\
\hline Average & $\mathbf{1 . 2 6}$ & $\mathbf{1 . 4 3}$ & $\mathbf{1 . 3 7}$ & $\mathbf{1 . 5 4}$ & $\mathbf{1 . 4 2}$ & $\mathbf{1 . 6 3}$ & $\mathbf{1 . 5 1}$ \\
\hline SD & $\mathbf{0 . 0 2}$ & $\mathbf{0 . 0 4}$ & $\mathbf{0 . 0 2}$ & $\mathbf{0 . 0 2}$ & $\mathbf{0 . 0 2}$ & $\mathbf{0 . 0 7}$ & $\mathbf{0 . 0 2}$ \\
\hline & & & & & & & \\
\hline Sporopollenin Analogue \# & $\mathbf{1 3}$ & $\mathbf{1 4}$ & $\mathbf{1 5}$ & $\mathbf{1 6}$ & $\mathbf{1 7}$ & $\mathbf{1 8}$ & $\mathbf{2 6}$ \\
\hline Sample 1 & 2.11 & 1.78 & 1.69 & 1.18 & 1.15 & 1.21 & 1.42 \\
\hline Sample 2 & 1.97 & 1.66 & 1.73 & 1.17 & 1.10 & 1.16 & 1.28 \\
\hline Sample 3 & 1.93 & 1.76 & 1.66 & 1.12 & 1.28 & 1.18 & 1.48 \\
\hline Average & $\mathbf{2 . 0 0}$ & $\mathbf{1 . 7 4}$ & $\mathbf{1 . 6 9}$ & $\mathbf{1 . 1 6}$ & $\mathbf{1 . 1 8}$ & $\mathbf{1 . 1 9}$ & $\mathbf{1 . 3 9}$ \\
\hline SD & $\mathbf{0 . 1 0}$ & $\mathbf{0 . 0 6}$ & $\mathbf{0 . 0 3}$ & $\mathbf{0 . 0 3}$ & $\mathbf{0 . 0 9}$ & $\mathbf{0 . 0 3}$ & $\mathbf{0 . 1 0}$ \\
\hline
\end{tabular}


Table S8. Weight swelling ratios (dimensionless) of synthetic sporopollenin analogues

\begin{tabular}{|l|l|l|l|l|l|l|l|}
\hline Sporopollenin Analogue \# & $\mathbf{6}$ & $\mathbf{7}$ & $\mathbf{8}$ & $\mathbf{9}$ & $\mathbf{1 0}$ & $\mathbf{1 1}$ & $\mathbf{1 2}$ \\
\hline Sample 1 & 1.24 & 1.43 & 1.24 & 1.61 & 1.33 & 1.55 & 1.41 \\
\hline Sample 2 & 1.21 & 1.34 & 1.27 & 1.43 & 1.30 & 1.49 & 1.33 \\
\hline Sample 3 & 1.26 & 1.32 & 1.25 & 1.47 & 1.26 & 1.56 & 1.35 \\
\hline Average & $\mathbf{1 . 2 3}$ & $\mathbf{1 . 3 7}$ & $\mathbf{1 . 2 5}$ & $\mathbf{1 . 5 0}$ & $\mathbf{1 . 3 0}$ & $\mathbf{1 . 5 4}$ & $\mathbf{1 . 3 7}$ \\
\hline SD & $\mathbf{0 . 0 3}$ & $\mathbf{0 . 0 6}$ & $\mathbf{0 . 0 2}$ & $\mathbf{0 . 0 9}$ & $\mathbf{0 . 0 3}$ & $\mathbf{0 . 0 4}$ & $\mathbf{0 . 0 4}$ \\
\hline & & & & & & & \\
\hline Sporopollenin Analogue \# & $\mathbf{1 3}$ & $\mathbf{1 4}$ & $\mathbf{1 5}$ & $\mathbf{1 6}$ & $\mathbf{1 7}$ & $\mathbf{1 8}$ & $\mathbf{2 6}$ \\
\hline Sample 1 & 1.74 & 1.57 & 1.59 & 1.09 & 1.06 & 1.09 & 1.34 \\
\hline Sample 2 & 1.76 & 1.57 & 1.58 & 1.11 & 0.95 & 1.08 & 1.26 \\
\hline Sample 3 & 1.65 & 1.57 & 1.53 & 1.09 & 1.04 & 1.13 & 1.28 \\
\hline Average & $\mathbf{1 . 7 1}$ & $\mathbf{1 . 5 7}$ & $\mathbf{1 . 5 7}$ & $\mathbf{1 . 1 0}$ & $\mathbf{1 . 0 2}$ & $\mathbf{1 . 1 0}$ & $\mathbf{1 . 3 0}$ \\
\hline SD & $\mathbf{0 . 0 6}$ & $\mathbf{0 . 0 0}$ & $\mathbf{0 . 0 3}$ & $\mathbf{0 . 0 1}$ & $\mathbf{0 . 0 6}$ & $\mathbf{0 . 0 2}$ & $\mathbf{0 . 0 4}$ \\
\hline
\end{tabular}


Thermal Analysis

\section{Thermogravimetric Analysis (TGA)}

\section{General Method:}

Thermogravimetric analysis was conducted by Element Materials Technology in accordance with Element New Berlin Procedure PA-04 with all polymer samples disclosed herein. Samples were heated from $20{ }^{\circ} \mathrm{C}$ to $650{ }^{\circ} \mathrm{C}$ at a rate of $20{ }^{\circ} \mathrm{C} / \mathrm{min}$ under an atmosphere of nitrogen, cooled to $500{ }^{\circ} \mathrm{C}$, exposed to air, then heated to $800^{\circ} \mathrm{C}$ at a rate of $20^{\circ} \mathrm{C} / \mathrm{min}$. Three mass loss events were observed. The first, observed at $184-236{ }^{\circ} \mathrm{C}$ in nitrogen, corresponds to evolution of loss of low molecular weight (i.e. solvents). The second, observed at $440-464{ }^{\circ} \mathrm{C}$ in nitrogen, corresponds to decomposition of the base polymer. The third, observed at $519-540{ }^{\circ} \mathrm{C}$ in air, corresponds to combustion which left behind a particulate carbonaceous residue.

Table S9. Thermogravimetric analysis of synthetic sporopollenin analogues

\begin{tabular}{|c|c|c|c|c|c|c|c|}
\hline $\begin{array}{l}\text { Sporopollenin } \\
\text { Analogue \# }\end{array}$ & 6 & 7 & 8 & 9 & 10 & 11 & 12 \\
\hline Loss $1\left(\%,{ }^{\circ} \mathrm{C}\right)$ & $\begin{array}{l}33.4 \\
208\end{array}$ & $\begin{array}{l}33.3 \\
217\end{array}$ & $\begin{array}{l}34.4 \\
210\end{array}$ & $\begin{array}{l}38.1 \\
211\end{array}$ & $\begin{array}{l}37.2 \\
215\end{array}$ & $\begin{array}{l}35.0 \\
218\end{array}$ & $\begin{array}{l}34.9 \\
226\end{array}$ \\
\hline Loss $2\left(\%,{ }^{\circ} \mathrm{C}\right)$ & $\begin{array}{l}55.6 \\
454\end{array}$ & $\begin{array}{l}55.7 \\
455\end{array}$ & $\begin{array}{l}53.8 \\
455\end{array}$ & $\begin{array}{l}49.3 \\
446\end{array}$ & $\begin{array}{l}50.1 \\
451\end{array}$ & $\begin{array}{l}50.8 \\
452\end{array}$ & $\begin{array}{l}51.8, \\
451\end{array}$ \\
\hline Loss $3\left(\%,{ }^{\circ} \mathrm{C}\right)$ & $9.7,540$ & $9.6,535$ & $\begin{array}{l}10.4 \\
542\end{array}$ & $\begin{array}{l}11.0 \\
532\end{array}$ & $\begin{array}{l}11.1 \\
530\end{array}$ & $\begin{array}{l}11.4 \\
534\end{array}$ & $\begin{array}{l}10.8 \\
519\end{array}$ \\
\hline Residue (\%) & 1.3 & 1.5 & 1.3 & 1.6 & 1.6 & 2.7 & 2.5 \\
\hline $\begin{array}{l}\text { Sporopollenin } \\
\text { Analogue \# }\end{array}$ & 13 & 14 & 15 & 16 & 17 & 18 & 26 \\
\hline Loss $1\left(\%,{ }^{\circ} \mathrm{C}\right)$ & $\begin{array}{l}37.9 \\
205\end{array}$ & $\begin{array}{l}37.0 \\
210\end{array}$ & $\begin{array}{l}35.6, \\
203\end{array}$ & $\begin{array}{l}32.5 \\
220\end{array}$ & $\begin{array}{l}30.6 \\
226\end{array}$ & $\begin{array}{l}31.6 \\
218\end{array}$ & $\begin{array}{l}32.3 \\
189\end{array}$ \\
\hline Loss $2\left(\%,{ }^{\circ} \mathrm{C}\right)$ & $\begin{array}{l}50.1 \\
446\end{array}$ & $\begin{array}{l}50.6 \\
450\end{array}$ & $\begin{array}{l}53.2 \\
444\end{array}$ & $\begin{array}{l}56.8 \\
457\end{array}$ & $\begin{array}{l}60.8 \\
464\end{array}$ & $\begin{array}{l}61.7 \\
463\end{array}$ & $\begin{array}{l}56.5 \\
450\end{array}$ \\
\hline Loss $3\left(\%,{ }^{\circ} \mathrm{C}\right)$ & $\begin{array}{l}10.6 \\
535\end{array}$ & $\begin{array}{l}10.7 \\
535\end{array}$ & $9.0,522$ & $9.6,540$ & $7.9,544$ & $6.0,548$ & $9.8,530$ \\
\hline Residue (\%) & 1.4 & 1.6 & 2.2 & 1.1 & 0.7 & 0.7 & 1.3 \\
\hline
\end{tabular}

\section{Overall:}

Average Loss $1\left(\%,{ }^{\circ} \mathrm{C}\right): 34.6,213$ SD $\left(\%,{ }^{\circ} \mathrm{C}\right): 2.4,9.8$

Average Loss $3\left(\%,{ }^{\circ} \mathrm{C}\right): 9.8,535$ SD $\left(\%,{ }^{\circ} \mathrm{C}\right): 1.4,8.0$
Average Loss $2\left(\%,{ }^{\circ} \mathrm{C}\right): 54.1,453$

$\mathrm{SD}\left(\%,{ }^{\circ} \mathrm{C}\right): 4.0,5.9$

Average Residue (\%): 1.5

SD (\%): 0.6 
General Method:

Differential scanning calorimetry was conducted in triplicate by Element Materials Technology in accordance with Element New Berlin Procedure PA-06 with synthetic sporopollenin analogue 15. A three-step methodology was employed in which samples were heated from $-60{ }^{\circ} \mathrm{C}$ to $275{ }^{\circ} \mathrm{C}$, control cooled to $-60{ }^{\circ} \mathrm{C}$, then heated to $300{ }^{\circ} \mathrm{C}$ under an atmosphere of nitrogen. No significant thermal features were observed below $110^{\circ} \mathrm{C}$. Above $110^{\circ} \mathrm{C}$, volatilization of residual low molecular weight compounds (i.e. solvents, observed via TGA) interfered with observations. Subsequent modulated DSC focused below $110^{\circ} \mathrm{C}$ was performed by heating samples from $-60^{\circ} \mathrm{C}$ to $110^{\circ} \mathrm{C}$ at an underlying heating rate of $3{ }^{\circ} \mathrm{C} / \mathrm{min}$ which was modulated $\pm 1{ }^{\circ} \mathrm{C}$ every $60 \mathrm{~s}$. 


\section{Supplementary Figures}

Figure S1. The ${ }^{1} \mathrm{H}$ NMR spectrum of compound S6

Figure S2. The ${ }^{1} \mathrm{H}$ NMR spectrum of compound $\mathbf{S 7}$

Figure S3. The ${ }^{1} \mathrm{H}$ NMR spectrum of compound 21

Figure S4. The ${ }^{1} \mathrm{H}$ NMR spectrum of compound 22

Figure S5. The ${ }^{1} \mathrm{H}$ NMR spectrum of compound $\mathbf{2 3}$

Figure S6. The ${ }^{1} \mathrm{H}$ NMR spectrum of compound 24

Figure S7. The ${ }^{1} \mathrm{H}$ NMR spectrum of compounds $\mathbf{S 9}$ and $\mathbf{S 1 0}$

Figure S8. The ${ }^{1} \mathrm{H}$ NMR spectrum of compounds S11 and S12

Figure S9. The ${ }^{1} \mathrm{H}$ NMR spectrum of compound $\mathbf{2 5}$

Figure S10. The ${ }^{13} \mathrm{C}$ NMR spectrum of compound 25

Figure S11. The ${ }^{13} \mathrm{C}$ MAS ssNMR spectrum of authentic $P$. rigida sporopollenin

Figure S12. The ${ }^{13} \mathrm{C}$ MAS ssNMR spectrum of simplified sporopollenin analogue 15

Figure S13. The ${ }^{13} \mathrm{C}$ MAS ssNMR spectrum of synthetic linker sporopollenin analogue 26

Figure S14. Overlaid ${ }^{13} \mathrm{C}$ MAS ssNMR spectra of authentic $P$. rigida sporopollenin (blue), simplified sporopollenin analogue 15 (green), and synthetic linker sporopollenin analogue 26 (red)

Figure S15. The IR spectrum of authentic $P$. rigida sporopollenin

Figure S16. The IR spectrum of simplified sporopollenin analogue $\mathbf{1 5}$

Figure S17. The IR spectrum of synthetic linker sporopollenin analogue $\mathbf{2 6}$

Figure S18. Overlaid IR spectra of authentic $P$. rigida sporopollenin (blue), simplified sporopollenin analogue $\mathbf{1 5}$ (green), and synthetic linker sporopollenin analogue 26 (red)

Figure S19. The base peak chromatograms of thioacidolyzed authentic $P$. rigida sporopollenin (red), simplified sporopollenin analogue 15 (blue), and synthetic linker sporopollenin analogue $\mathbf{2 6}$ (black)

Figure S20. The extracted-ion chromatograms of thioacidolyzed authentic $P$. rigida sporopollenin (red), simplified sporopollenin analogue 15 (blue), and synthetic linker sporopollenin analogue $\mathbf{2 6}$ (black)

Figure S21. Compressive stress-strain curve for synthetic sporopollenin analogue 6 Figure S22. Compressive stress-strain curve for synthetic sporopollenin analogue 7 Figure S23. Compressive stress-strain curve for synthetic sporopollenin analogue 8 Figure S24. Compressive stress-strain curve for synthetic sporopollenin analogue 9 Figure S25. Compressive stress-strain curve for synthetic sporopollenin analogue $\mathbf{1 0}$ Figure S26. Compressive stress-strain curve for synthetic sporopollenin analogue 11 Figure S27. Compressive stress-strain curve for synthetic sporopollenin analogue $\mathbf{1 2}$ Figure S28. Compressive stress-strain curve for synthetic sporopollenin analogue $\mathbf{1 3}$ Figure S29. Compressive stress-strain curve for synthetic sporopollenin analogue 14 Figure S30. Compressive stress-strain curve for synthetic sporopollenin analogue $\mathbf{1 5}$ Figure S31. Compressive stress-strain curve for synthetic sporopollenin analogue 16 Figure S32. Compressive stress-strain curve for synthetic sporopollenin analogue 17 Figure S33. Compressive stress-strain curve for synthetic sporopollenin analogue $\mathbf{1 8}$ Figure S34. Compressive stress-strain curve for synthetic sporopollenin analogue 26

Figure S35. Relationship between compressive modulus and linker carbon count in simplified (black) and substituted (red) synthetic linker sporopollenin analogues 
Figure S36. Relationship between compressive modulus and linker density in simplified (black) and substituted (red) synthetic linker sporopollenin analogues

Figure S37. Relationship between compressive modulus and PVA $\mathrm{MW}_{\text {avg }}$ in simplified (black) and substituted (red) synthetic linker sporopollenin analogues

Figure S38. Relationship between compressive hysteresis ratio and linker carbon count in simplified (black) and substituted (red) synthetic linker sporopollenin analogues

Figure S39. Relationship between compressive hysteresis ratio and linker density in simplified (black) and substituted (red) synthetic linker sporopollenin analogues

Figure S40. Relationship between compressive hysteresis ratio and PVA MW avg in simplified (black) and substituted (red) synthetic linker sporopollenin analogues

Figure S41. Tensile stress-strain curve for synthetic sporopollenin analogue 12 at 1.0 (red) and $0.1 \mathrm{~min}^{-1}$ (black) loading rates

Figure S42. Tensile stress-strain curve for synthetic sporopollenin analogue 12 with cyclic (red) and monotonic (black) loading

Figure S43. Tensile stress-time relaxation curve for synthetic sporopollenin analogue 12

Figure S44. The TGA thermogram for synthetic sporopollenin analogue 6

Figure S45. The TGA thermogram for synthetic sporopollenin analogue 7

Figure S46. The TGA thermogram for synthetic sporopollenin analogue 8

Figure S47. The TGA thermogram for synthetic sporopollenin analogue 9

Figure S48. The TGA thermogram for synthetic sporopollenin analogue $\mathbf{1 0}$

Figure S49. The TGA thermogram for synthetic sporopollenin analogue 11

Figure S50. The TGA thermogram for synthetic sporopollenin analogue 12

Figure S51. The TGA thermogram for synthetic sporopollenin analogue $\mathbf{1 3}$

Figure S52. The TGA thermogram for synthetic sporopollenin analogue 14

Figure S53. The TGA thermogram for synthetic sporopollenin analogue $\mathbf{1 5}$

Figure S54. The TGA thermogram for synthetic sporopollenin analogue $\mathbf{1 6}$

Figure S55. The TGA thermogram for synthetic sporopollenin analogue 17

Figure S56. The TGA thermogram for synthetic sporopollenin analogue $\mathbf{1 8}$

Figure S57. The TGA thermogram for synthetic sporopollenin analogue $\mathbf{2 6}$

Figure S58. The DSC thermogram for synthetic sporopollenin analogue 15, first replicate

Figure S59. The DSC thermogram for synthetic sporopollenin analogue 15, second replicate

Figure S60. The DSC thermogram for synthetic sporopollenin analogue 15, third replicate

Figure S61. The DSC thermogram for synthetic sporopollenin analogue 15, all replicates

Figure S62. The modulated DSC thermogram for synthetic sporopollenin analogue $\mathbf{1 5}$ 


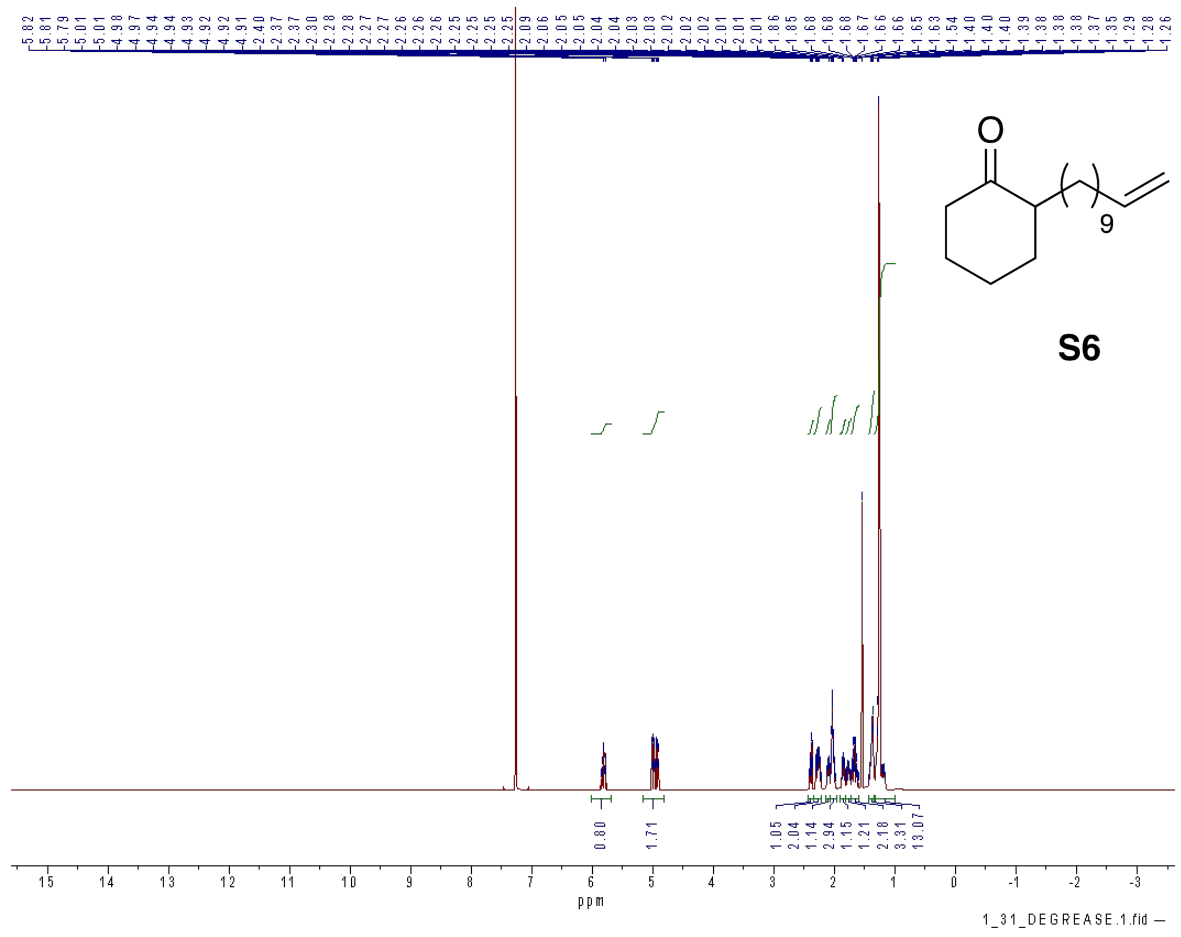

Figure S1. The ${ }^{1} \mathrm{H}$ NMR spectrum of compound S6

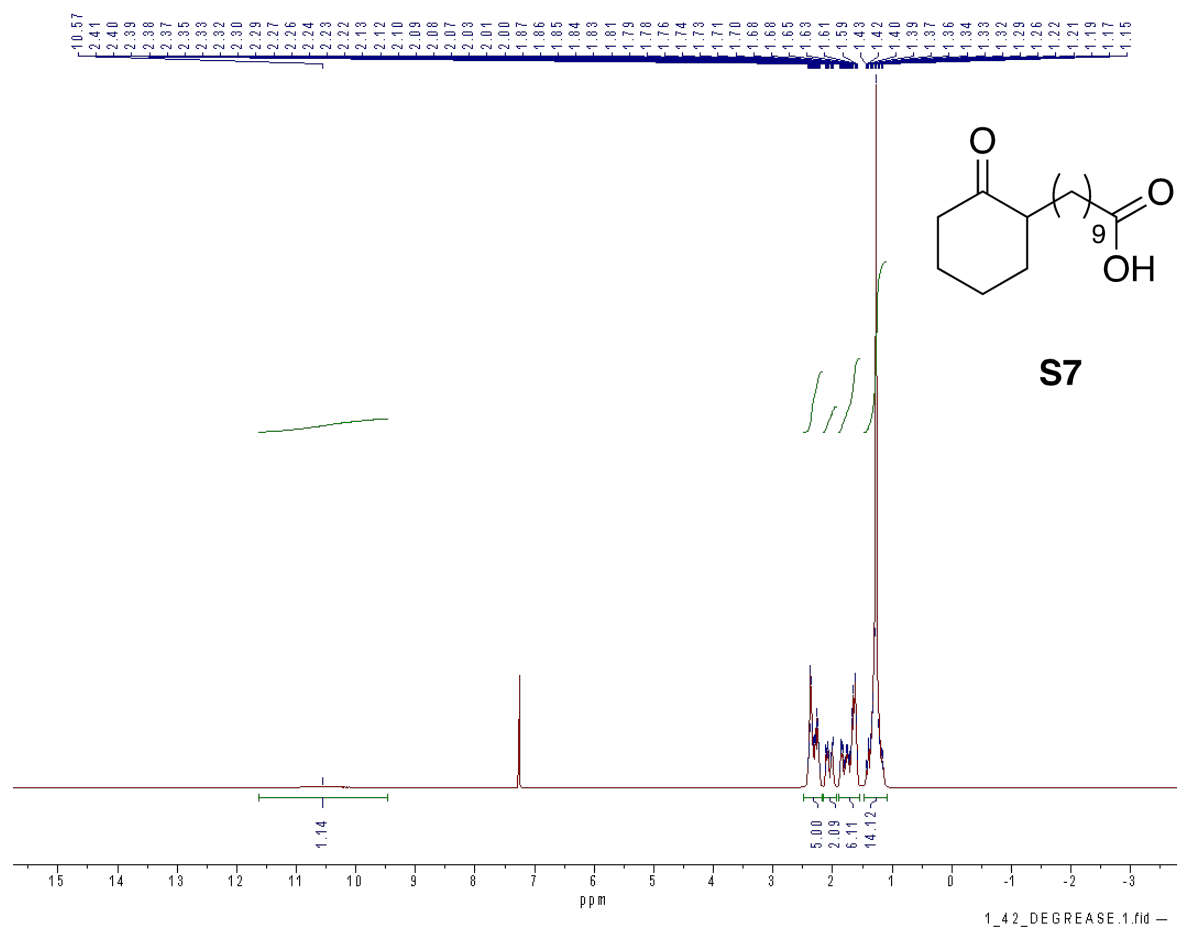

Figure S2. The ${ }^{1} \mathrm{H}$ NMR spectrum of compound S7 


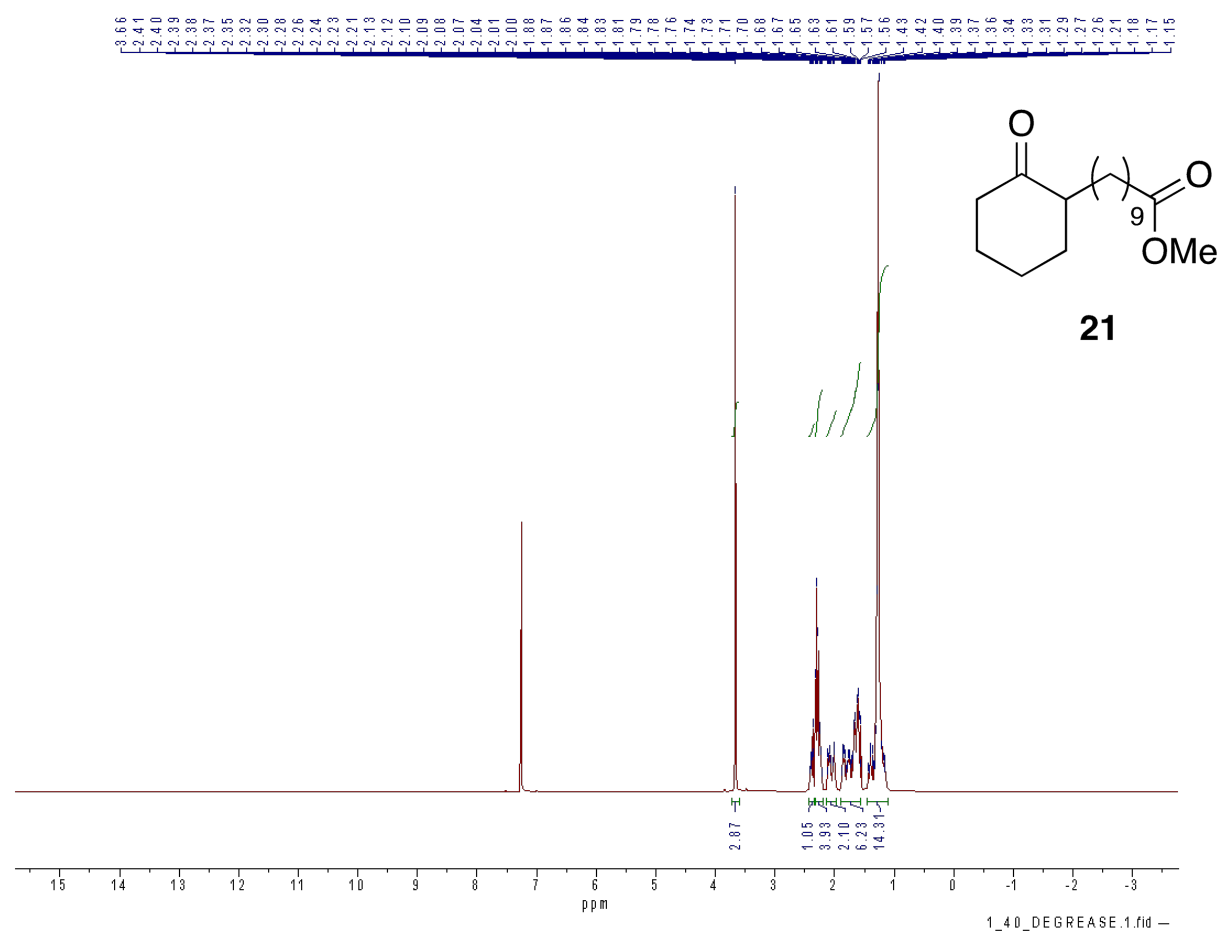

Figure S3. The ${ }^{1} \mathrm{H}$ NMR spectrum of compound 21

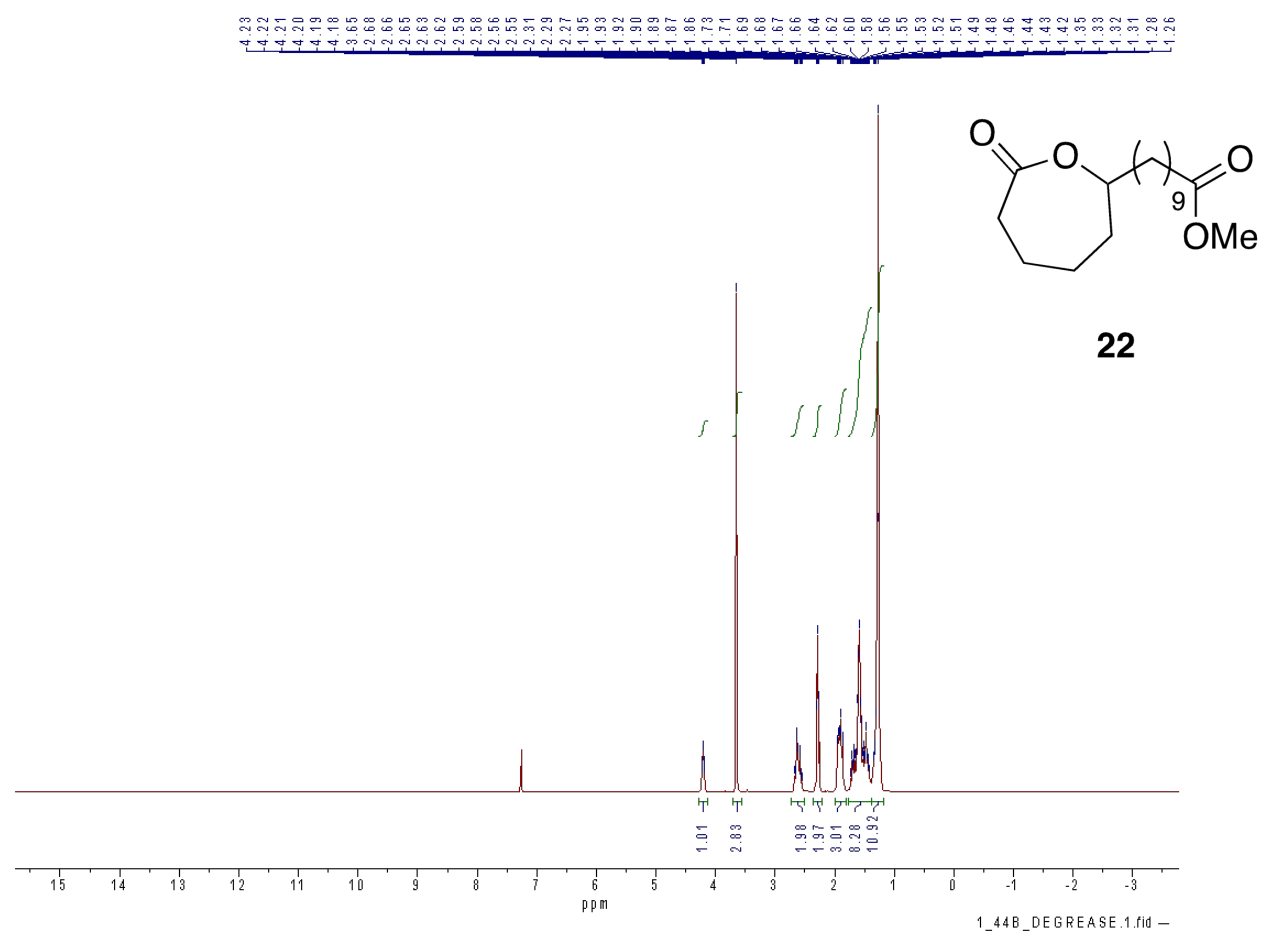

Figure S4. The ${ }^{1} \mathrm{H}$ NMR spectrum of compound 22 


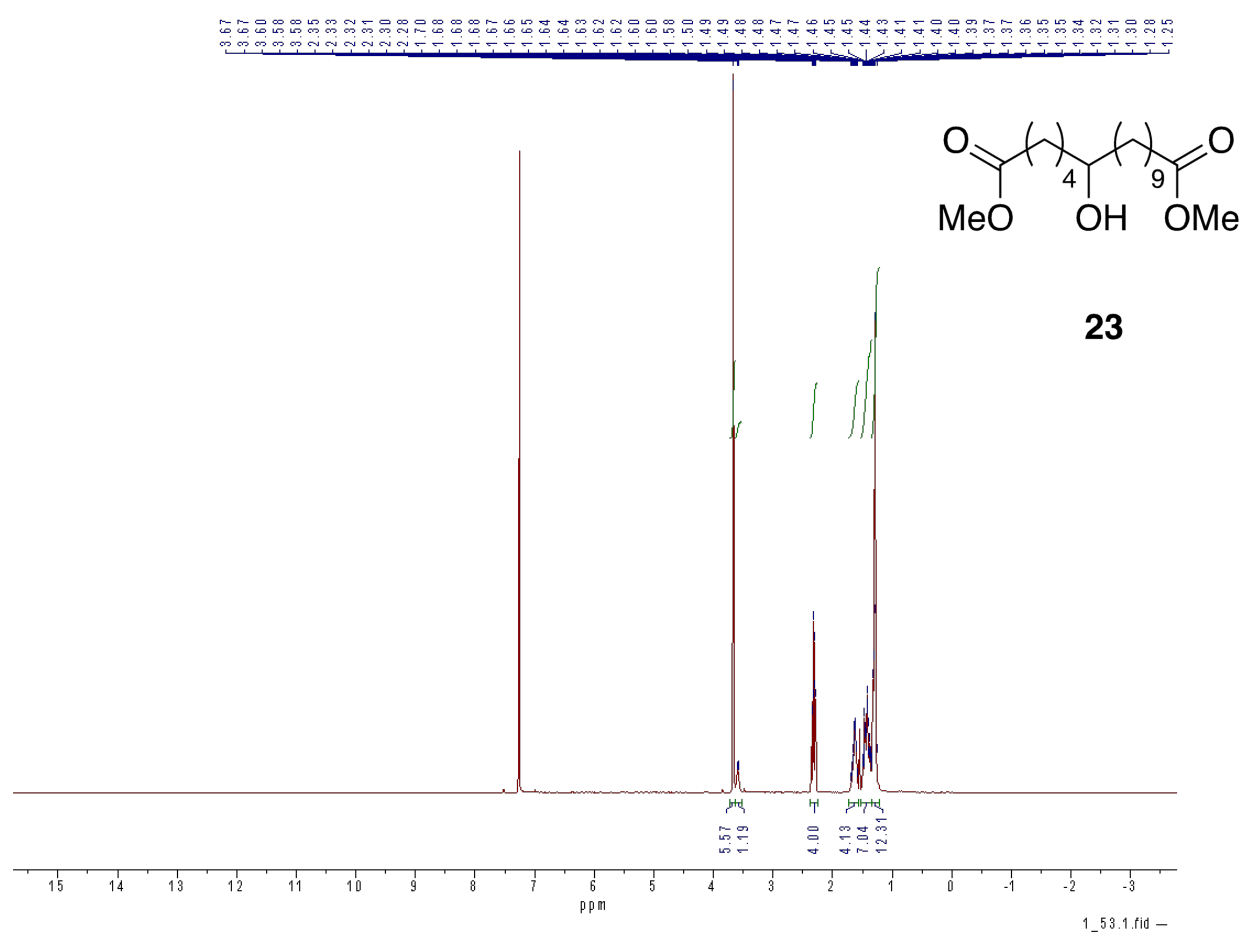

Figure S5. The ${ }^{1} \mathrm{H}$ NMR spectrum of compound 23

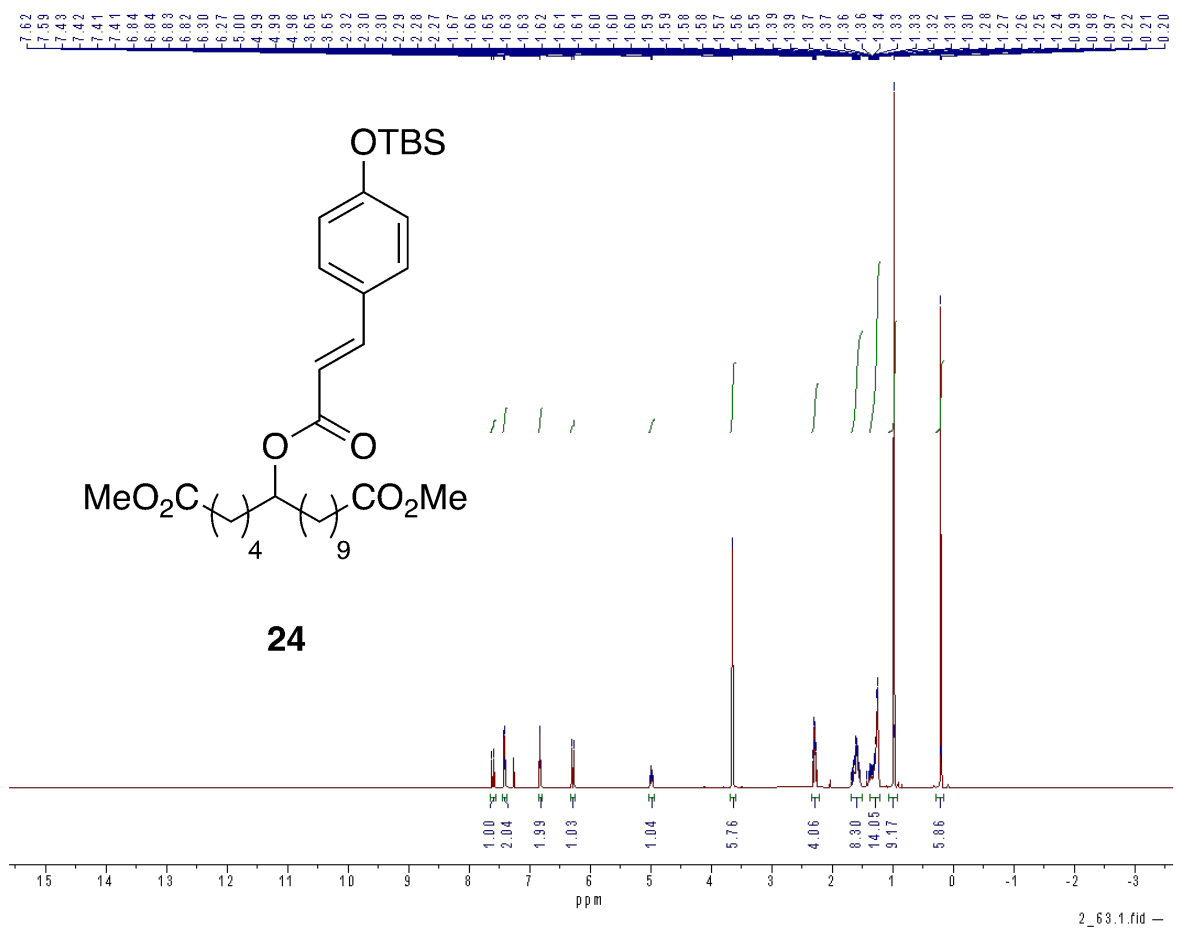

Figure S6. The ${ }^{1} \mathrm{H}$ NMR spectrum of compound 24 


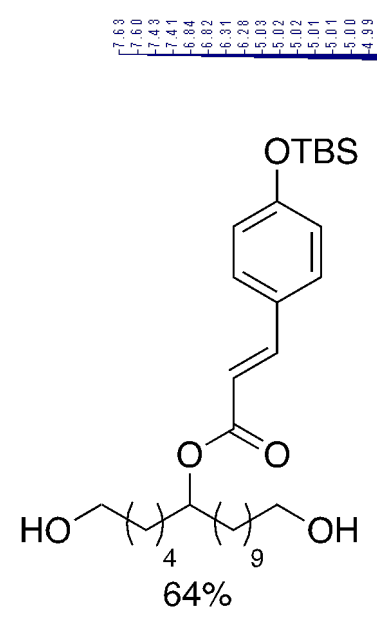

s9

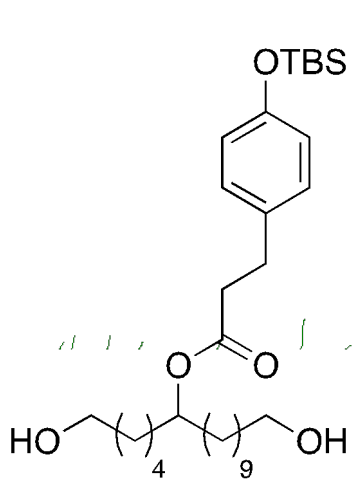

510

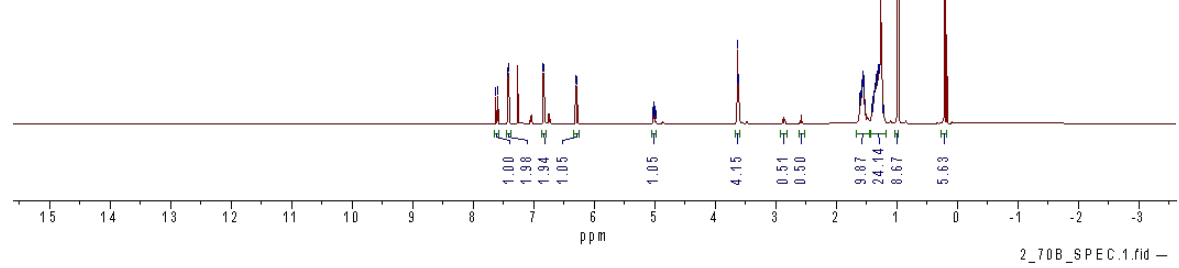

Figure S7. The ${ }^{1} \mathrm{H}$ NMR spectrum of compounds $\mathbf{S 9}$ and $\mathbf{S 1 0}$

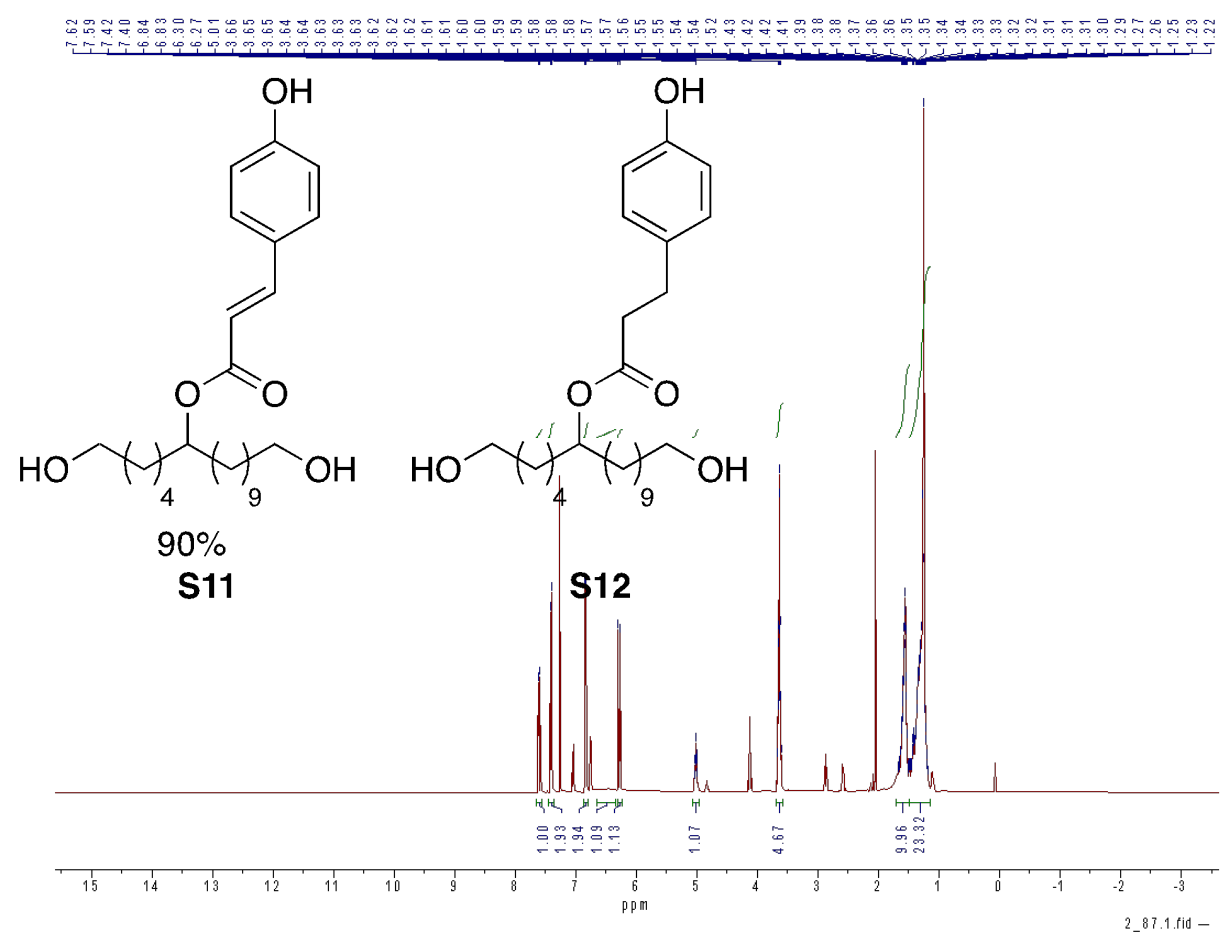

Figure S8. The ${ }^{1} \mathrm{H}$ NMR spectrum of compounds S11 and S12 


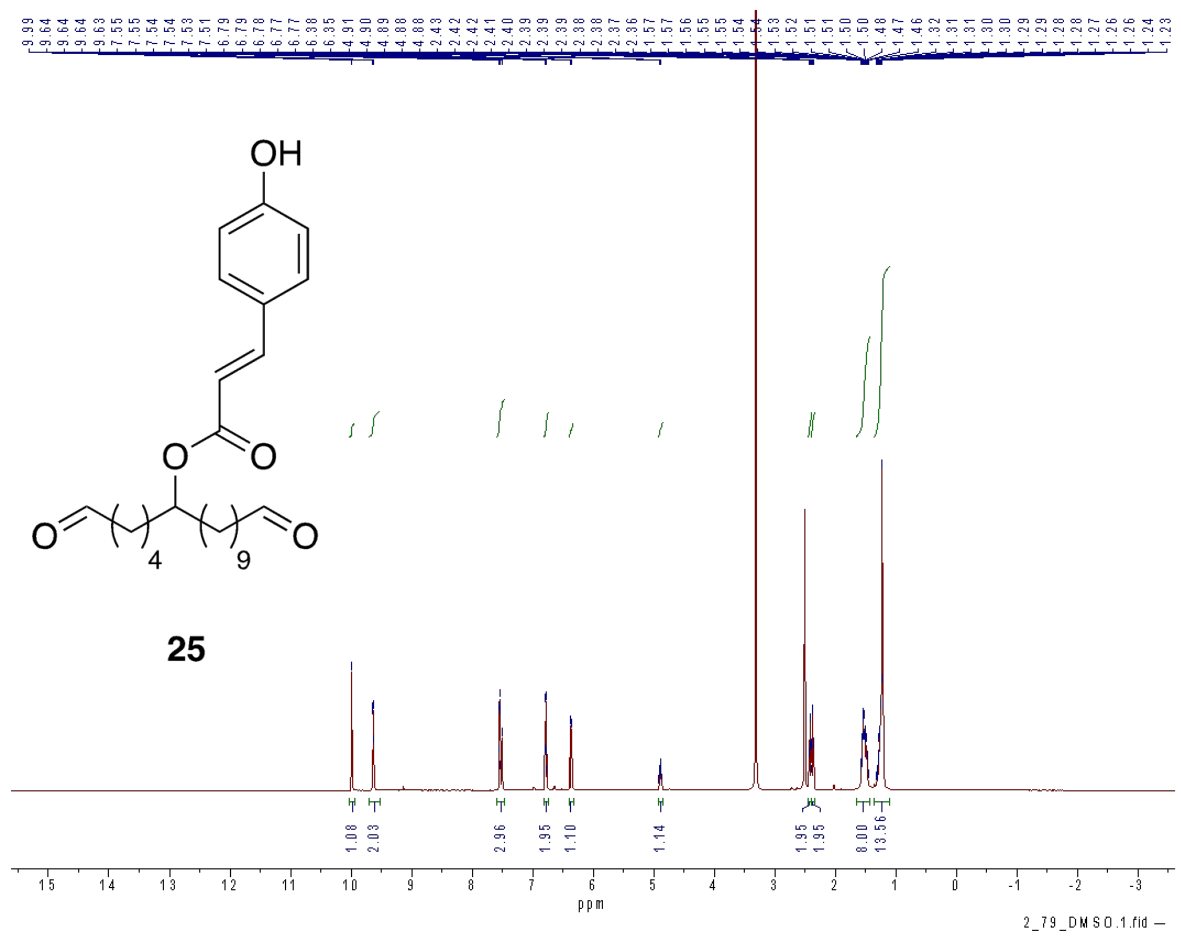

Figure S9. The ${ }^{1} \mathrm{H}$ NMR spectrum of compound 25
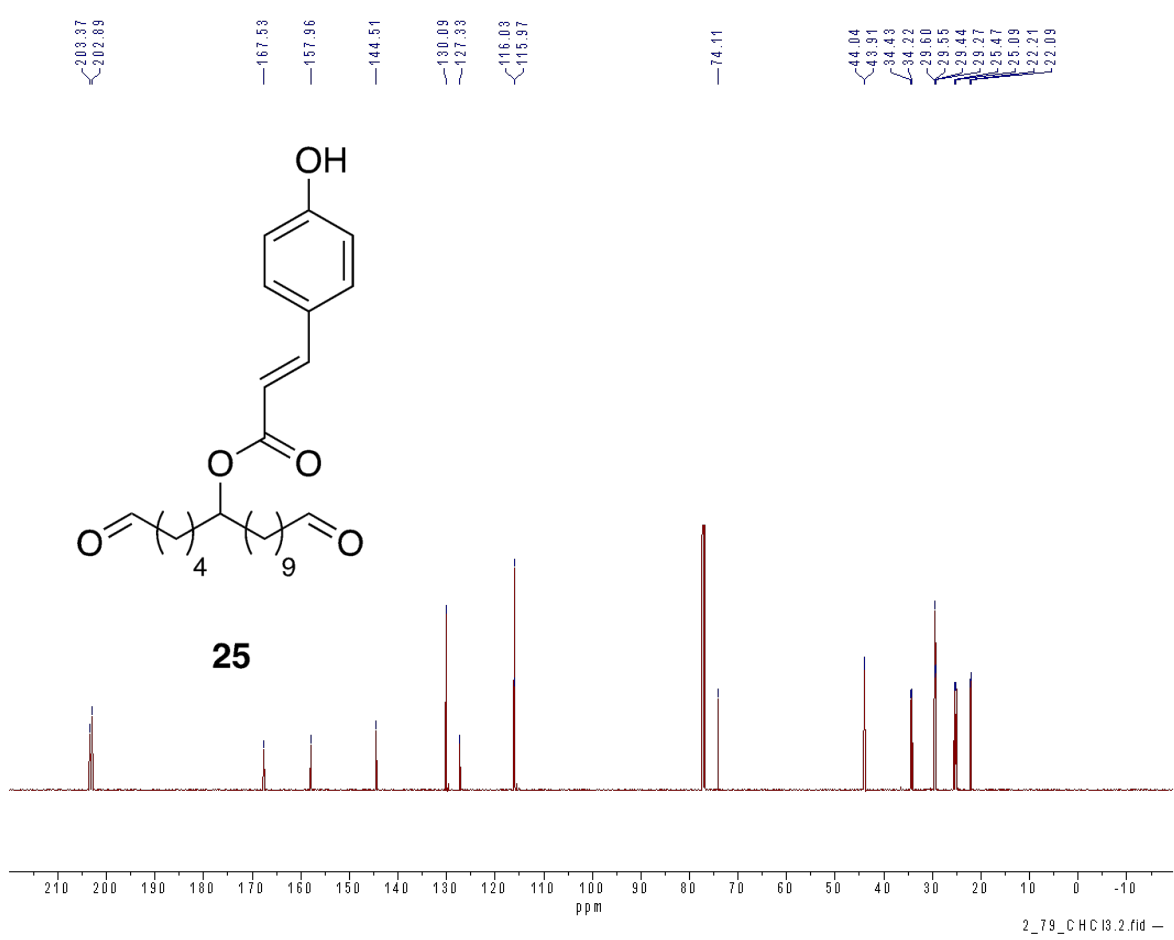

Figure S10. The ${ }^{13} \mathrm{C}$ NMR spectrum of compound 25 


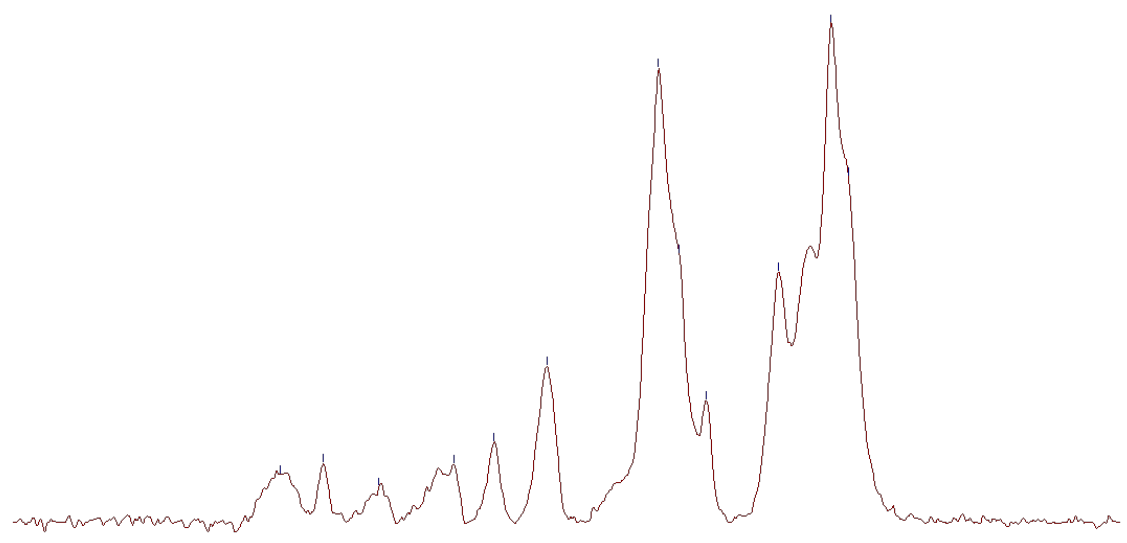

Figure S11. The ${ }^{13} \mathrm{C}$ MAS ssNMR spectrum of authentic $P$. rigida sporopollenin (9)

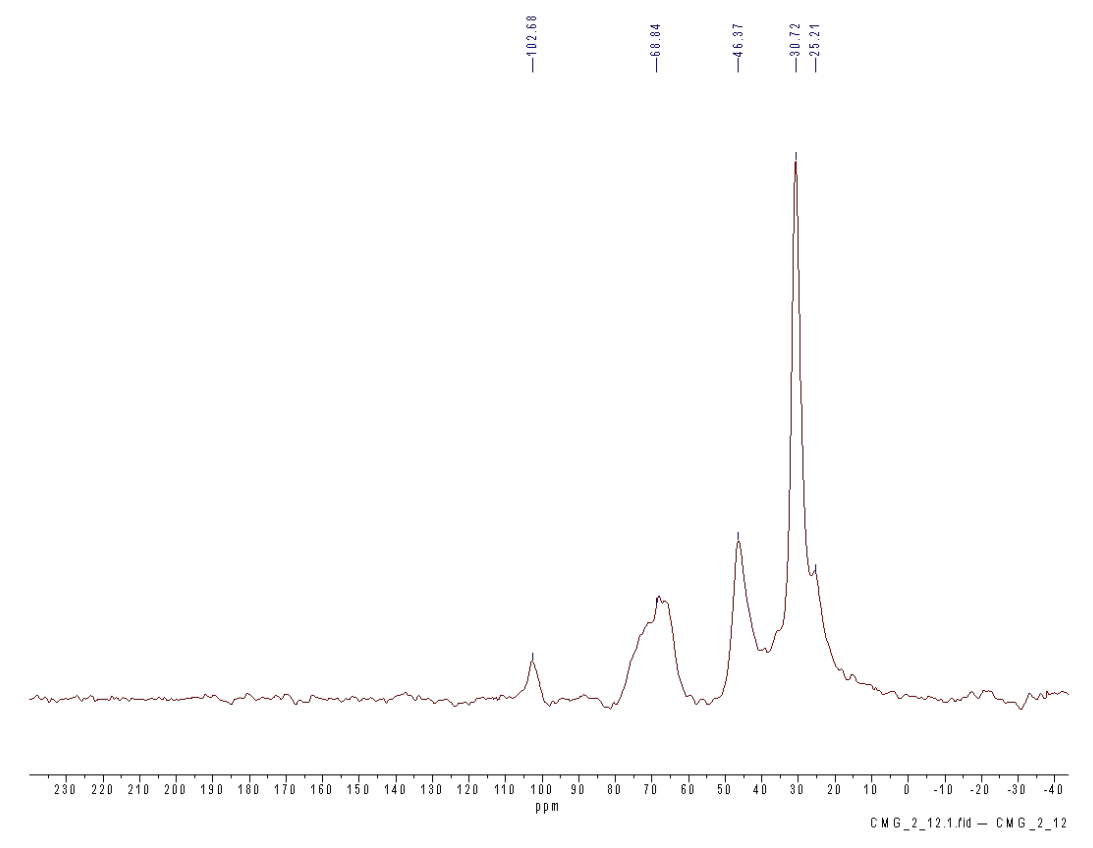

Figure S12. The $\overline{{ }^{13} \mathrm{C} \text { MAS ssNMR spectrum of simplified sporopollenin analogue } 15}$ 


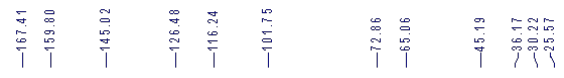

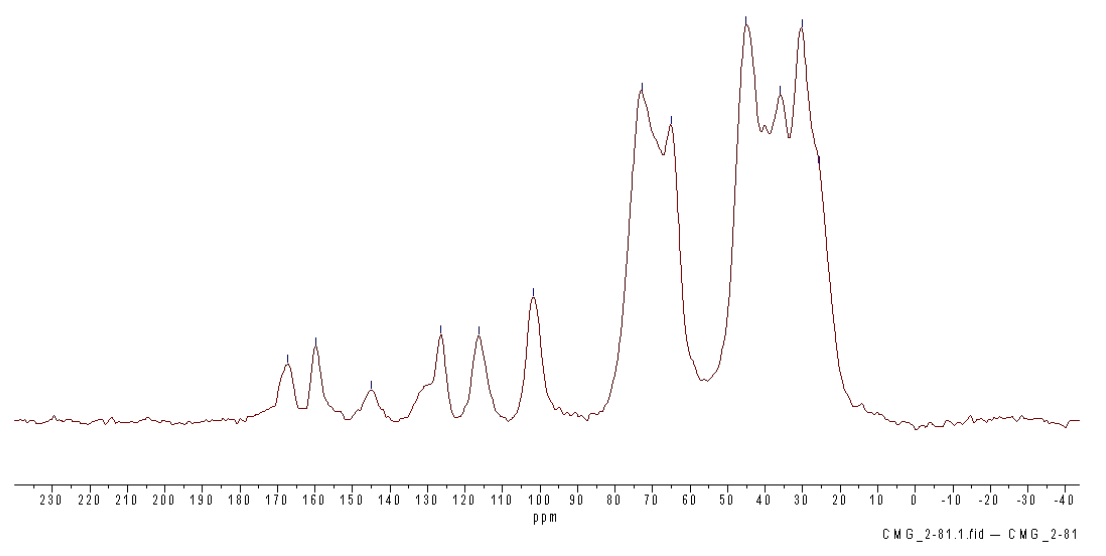

Figure S13. The ${ }^{13} \mathrm{C}$ MAS ssNMR spectrum of synthetic linker sporopollenin analogue $\mathbf{2 6}$
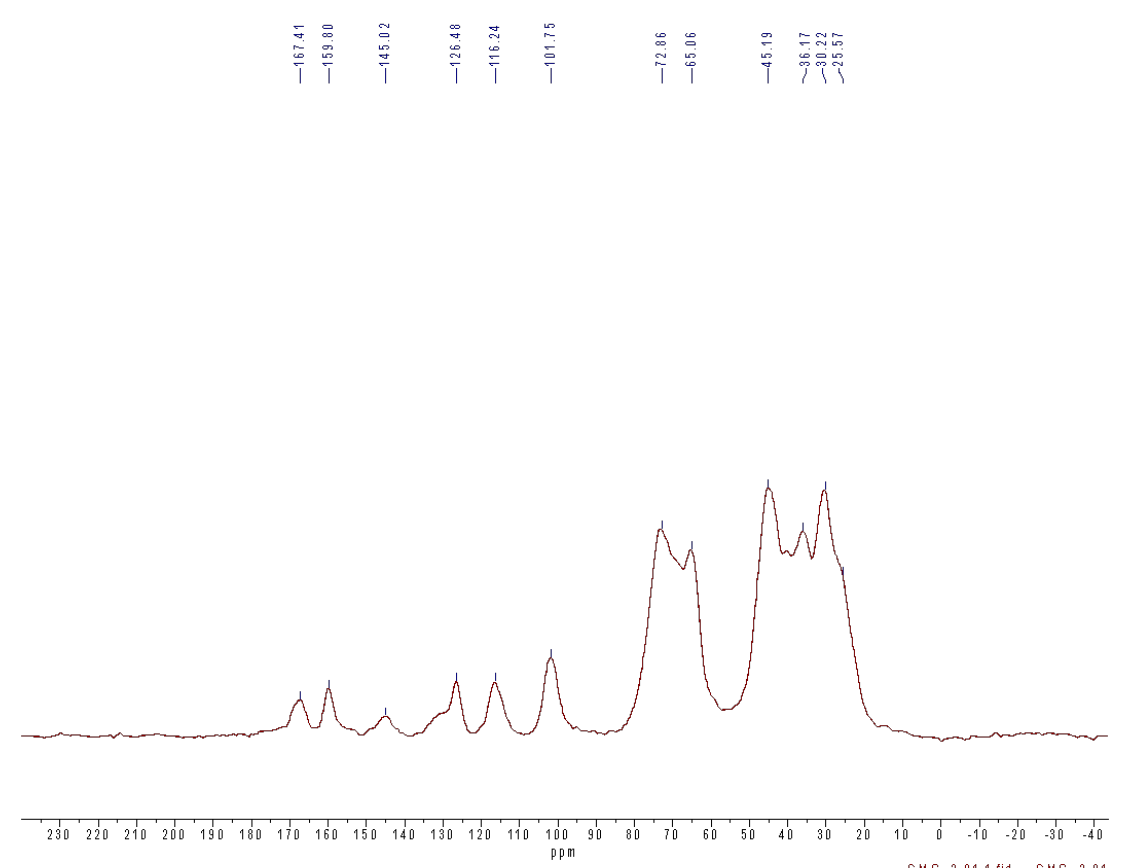

Figure S14. Overlaid ${ }^{13} \mathrm{C}$ MAS ssNMR spectra of authentic $P$. rigida sporopollenin (blue) (9), simplified sporopollenin analogue 15 (green), and synthetic linker sporopollenin analogue 26 (red) 


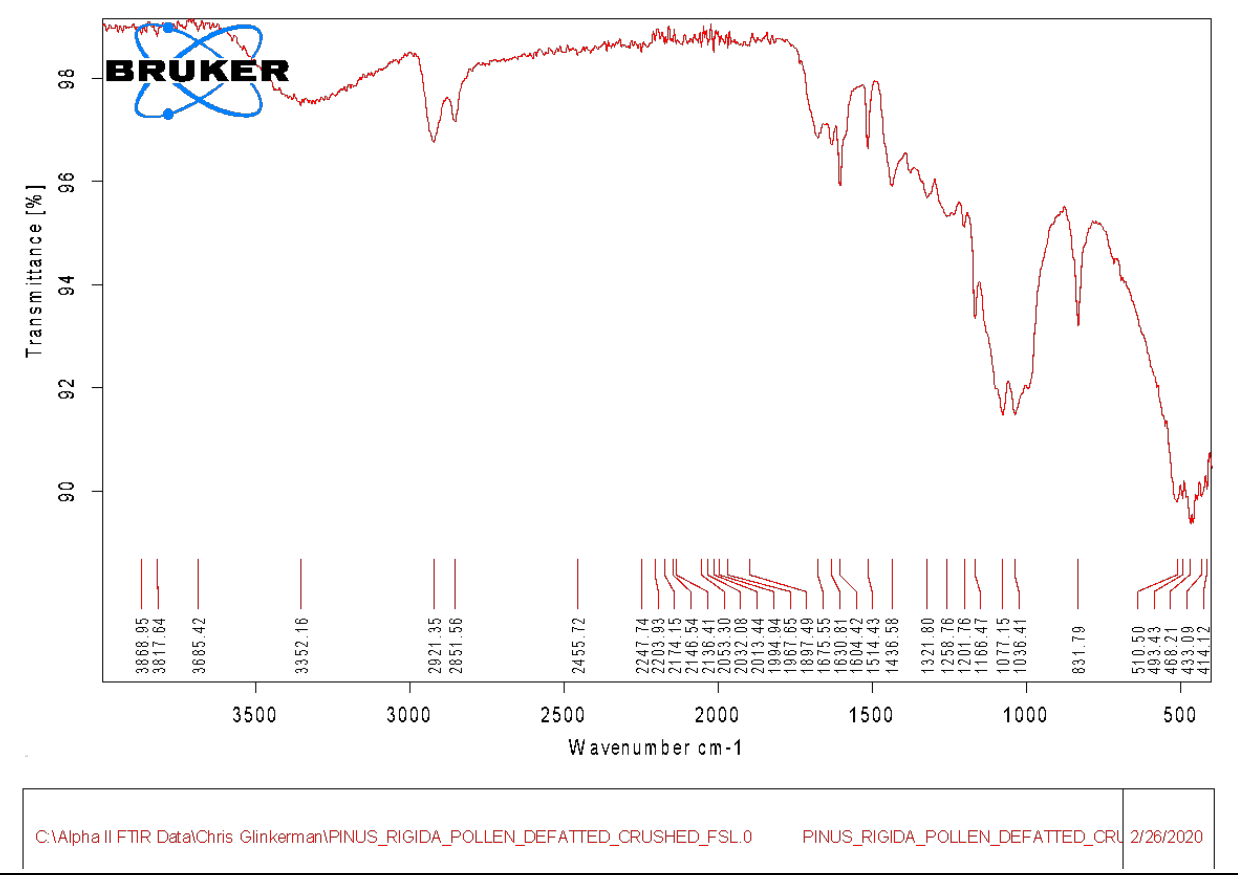

Figure S15. The IR spectrum of authentic $P$. rigida sporopollenin



Figure S16. The IR spectrum of simplified sporopollenin analogue 15 


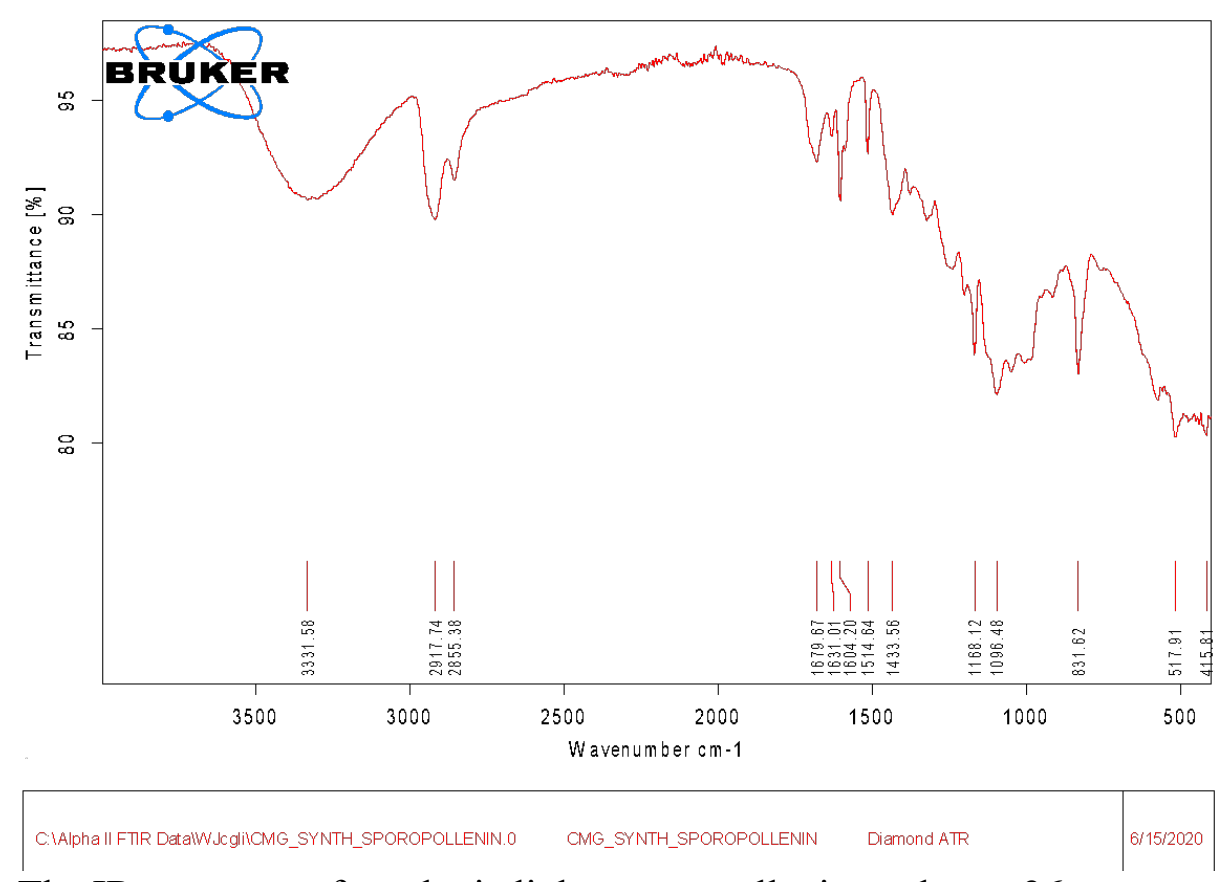

Figure S17. The IR spectrum of synthetic linker sporopollenin analogue 26

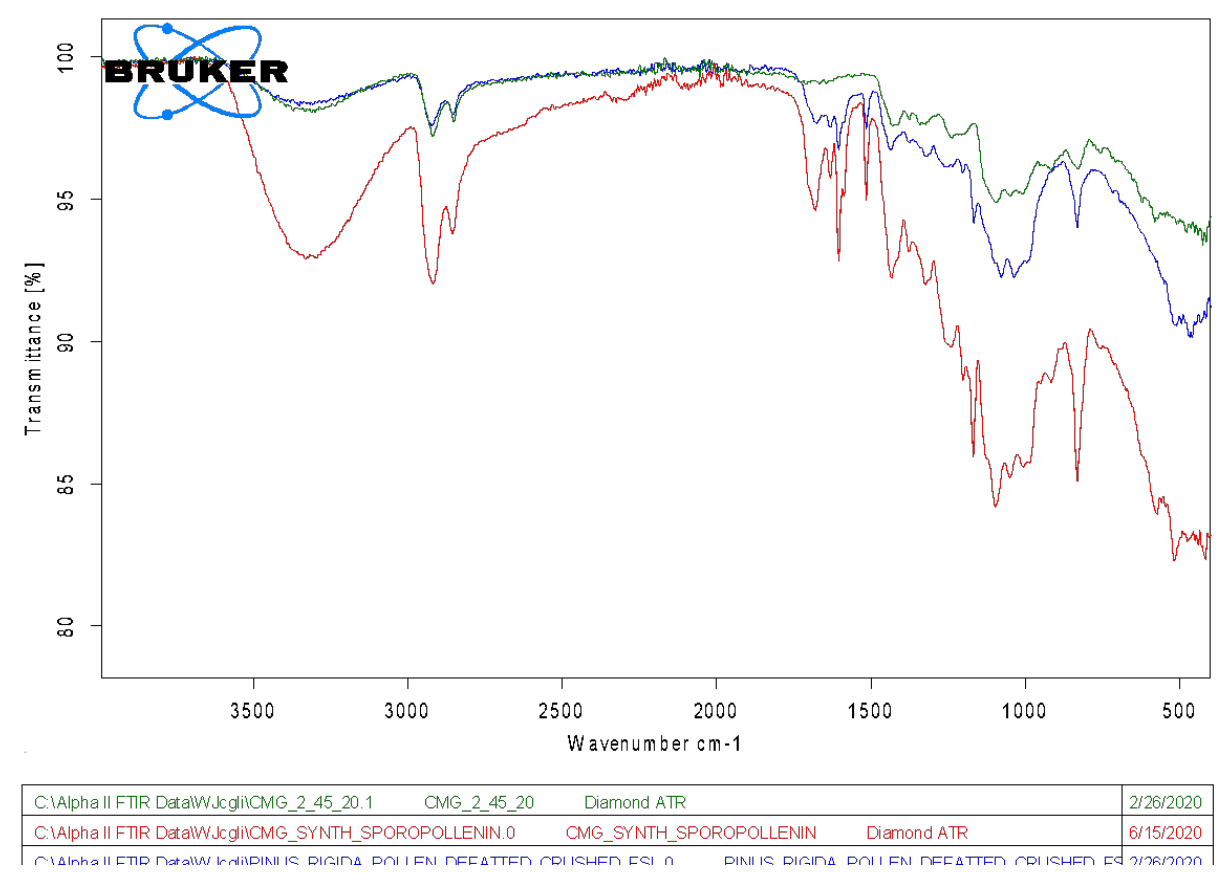

Figure S18. Overlaid IR spectra of authentic $P$. rigida sporopollenin (blue), simplified sporopollenin analogue 15 (green), and synthetic linker sporopollenin analogue $\mathbf{2 6}$ (red) 


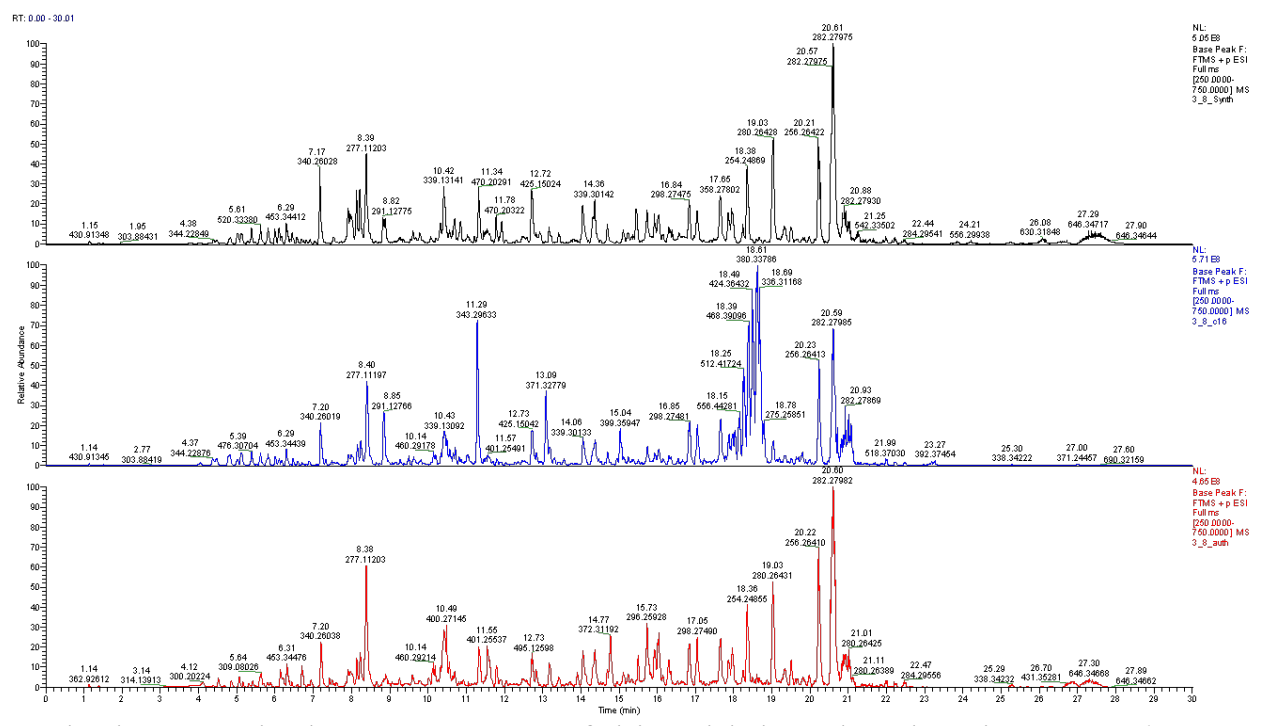

Figure S19. The base peak chromatograms of thioacidolyzed authentic $P$. rigida sporopollenin (red), simplified sporopollenin analogue $\mathbf{1 5}$ (blue), and synthetic linker sporopollenin analogue 26 (black)

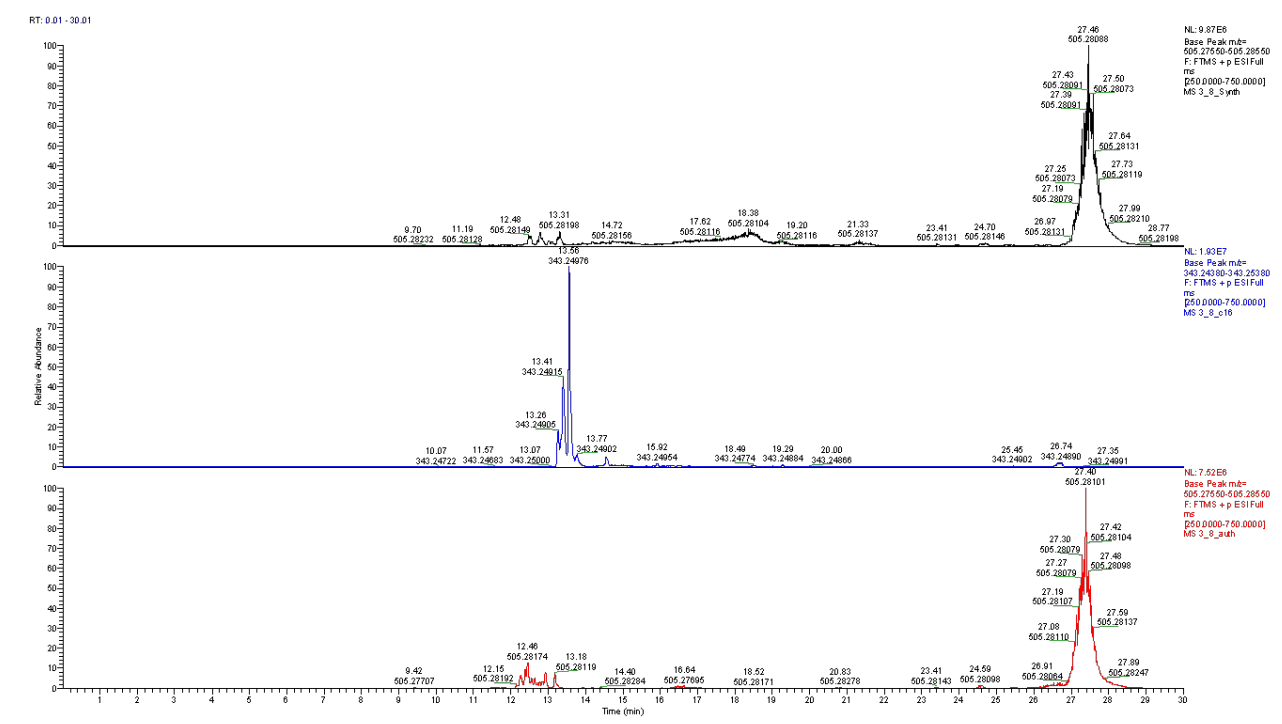

Figure S20. The extracted-ion chromatograms of thioacidolyzed authentic $P$. rigida sporopollenin (red), simplified sporopollenin analogue 15 (blue), and synthetic linker sporopollenin analogue $\mathbf{2 6}$ (black) 


\section{Stress vs. Strain}

Synthetic Sporopollenin 6

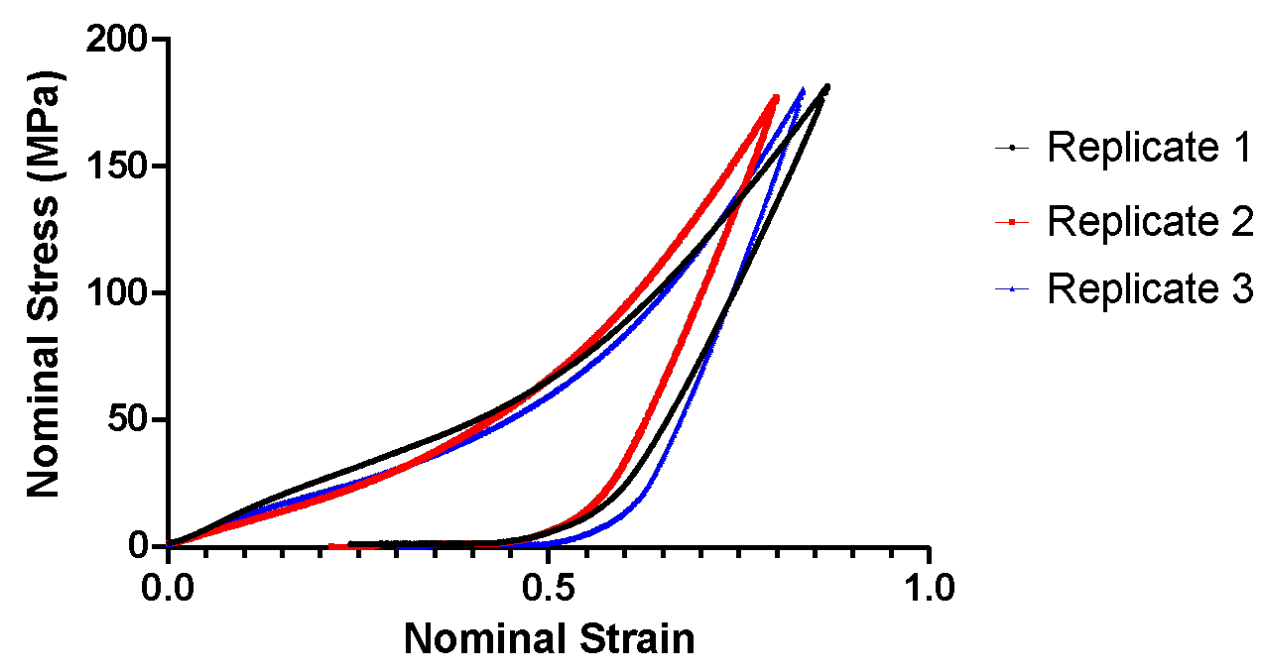

Figure S21. Compressive stress-strain curve for synthetic sporopollenin analogue 6

Stress vs. Strain

Synthetic Sporopollenin 7



Figure S22. Compressive stress-strain curve for synthetic sporopollenin analogue 7 


\section{Stress vs. Strain}

Synthetic Sporopollenin 8

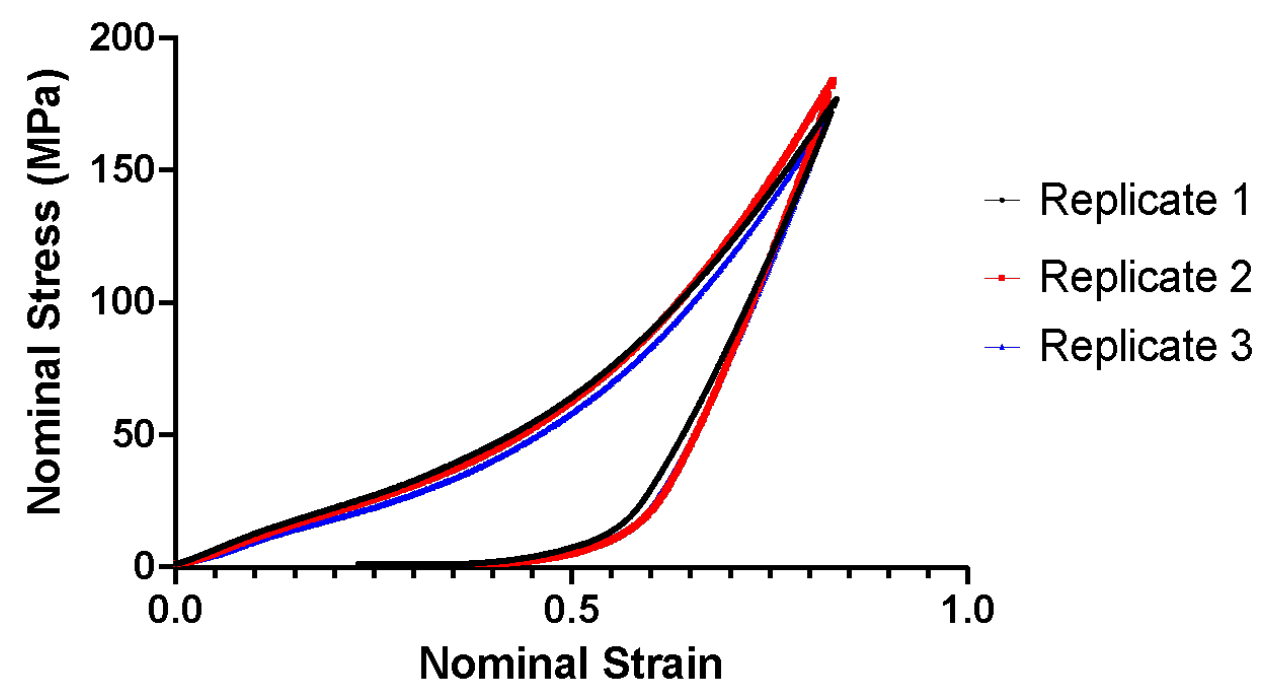

Figure S23. Compressive stress-strain curve for synthetic sporopollenin analogue 8

Stress vs. Strain

Synthetic Sporopollenin 9

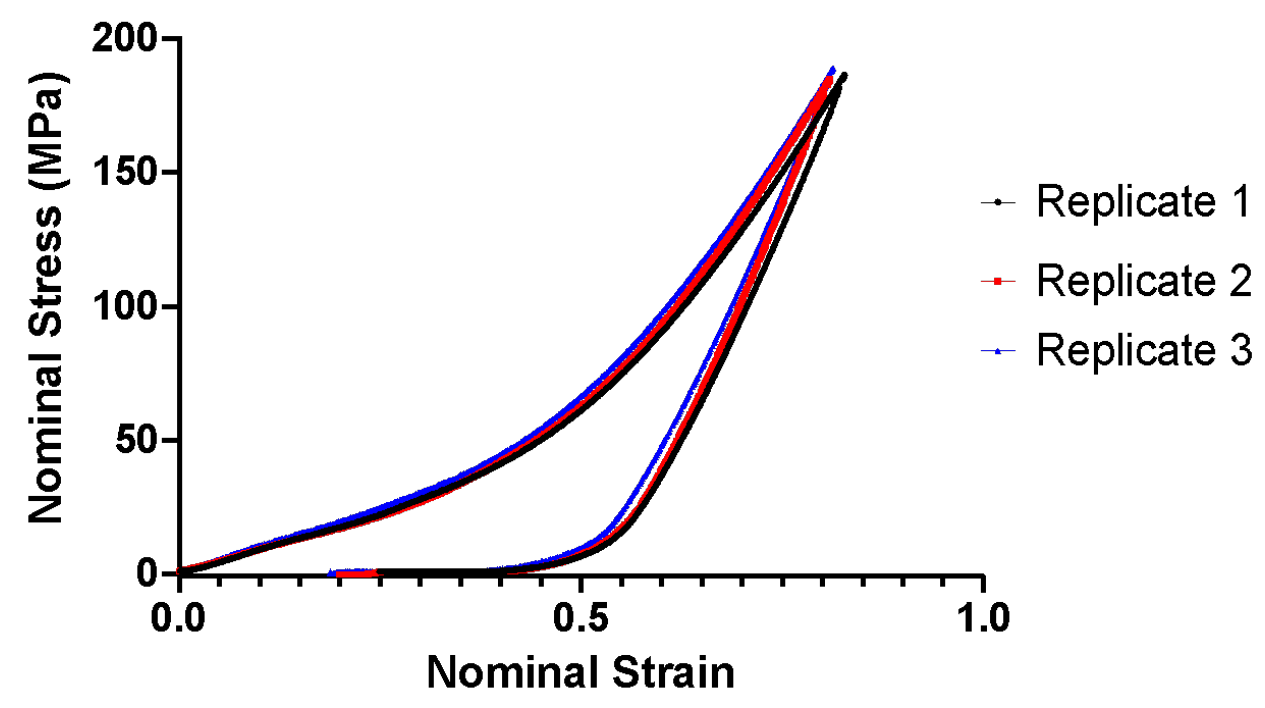

Figure S24. Compressive stress-strain curve for synthetic sporopollenin analogue 9 


\section{Stress vs. Strain}

Synthetic Sporopollenin 10

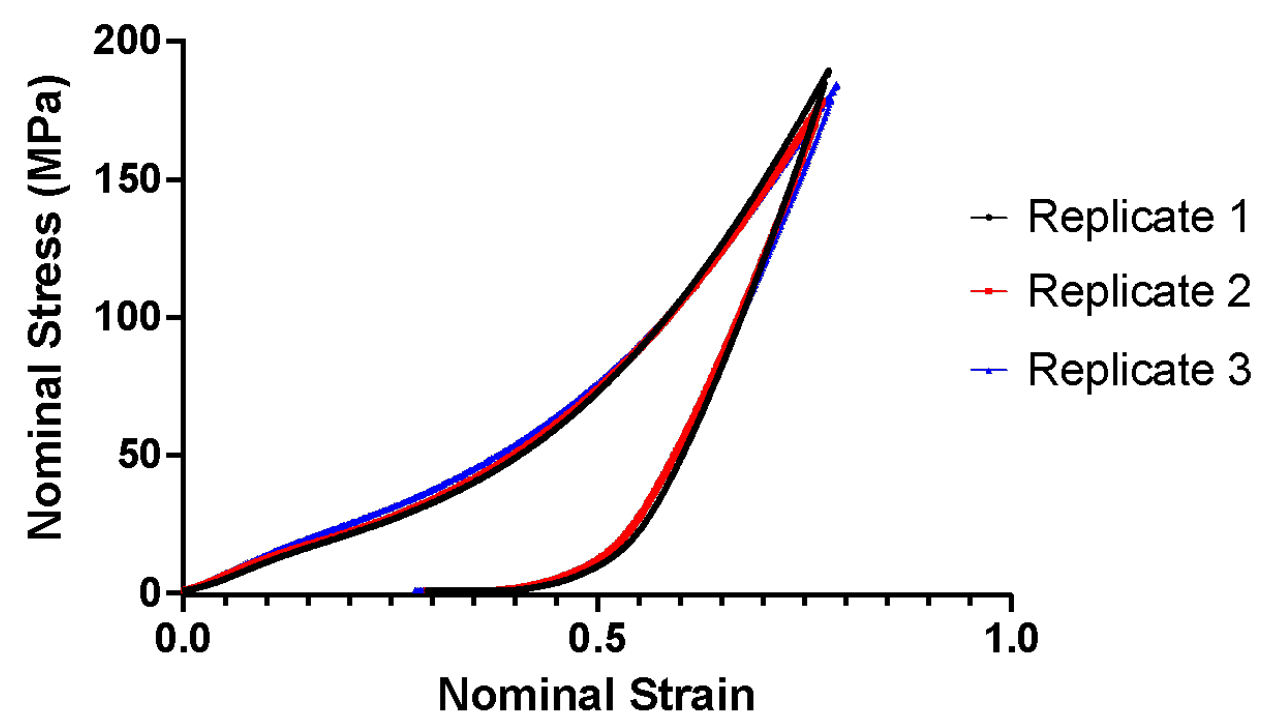

Figure S25. Compressive stress-strain curve for synthetic sporopollenin analogue $\mathbf{1 0}$

Stress vs. Strain

Synthetic Sporopollenin 11

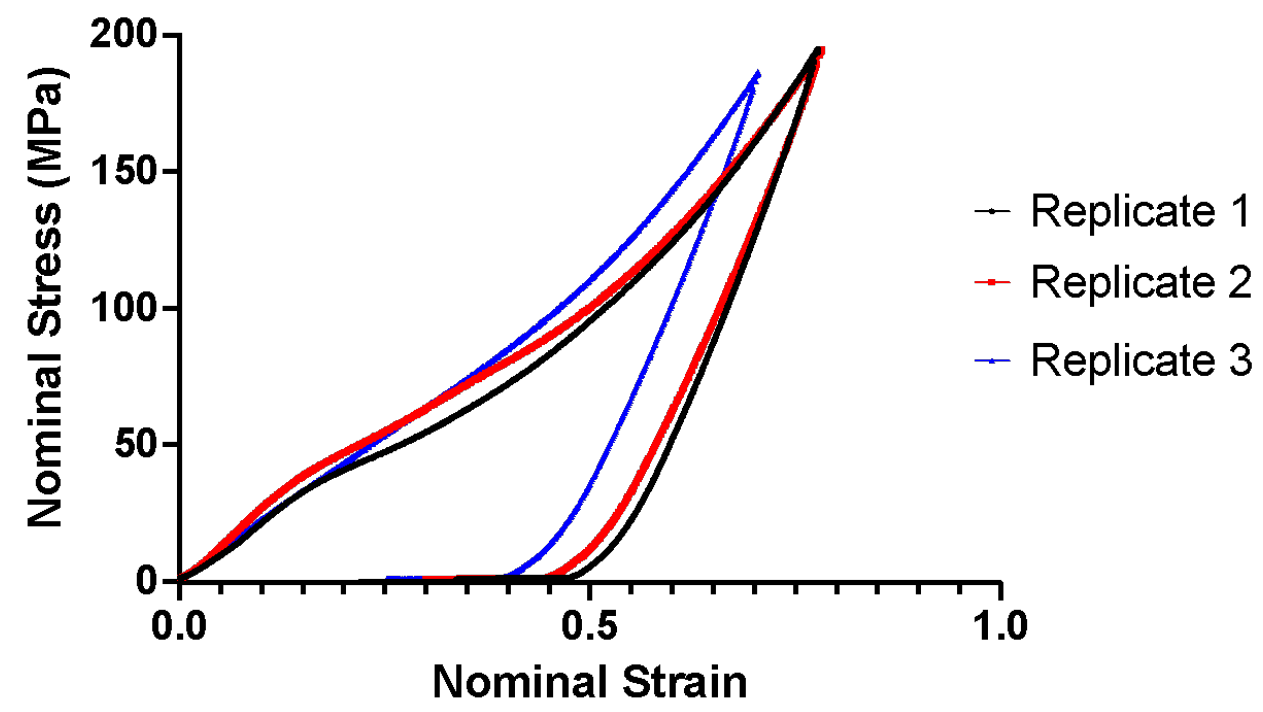

Figure S26. Compressive stress-strain curve for synthetic sporopollenin analogue 11 


\section{Stress vs. Strain}

Synthetic Sporopollenin 12

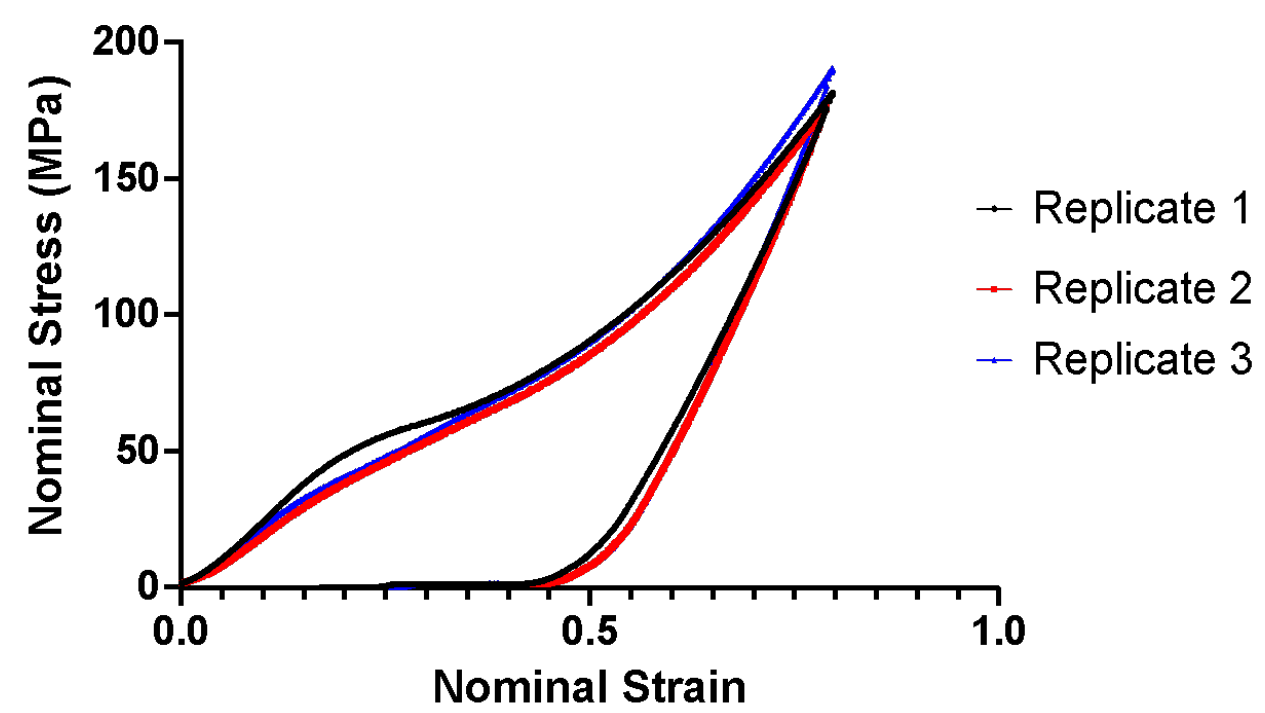

Figure S27. Compressive stress-strain curve for synthetic sporopollenin analogue 12

Stress vs. Strain

Synthetic Sporopollenin 13

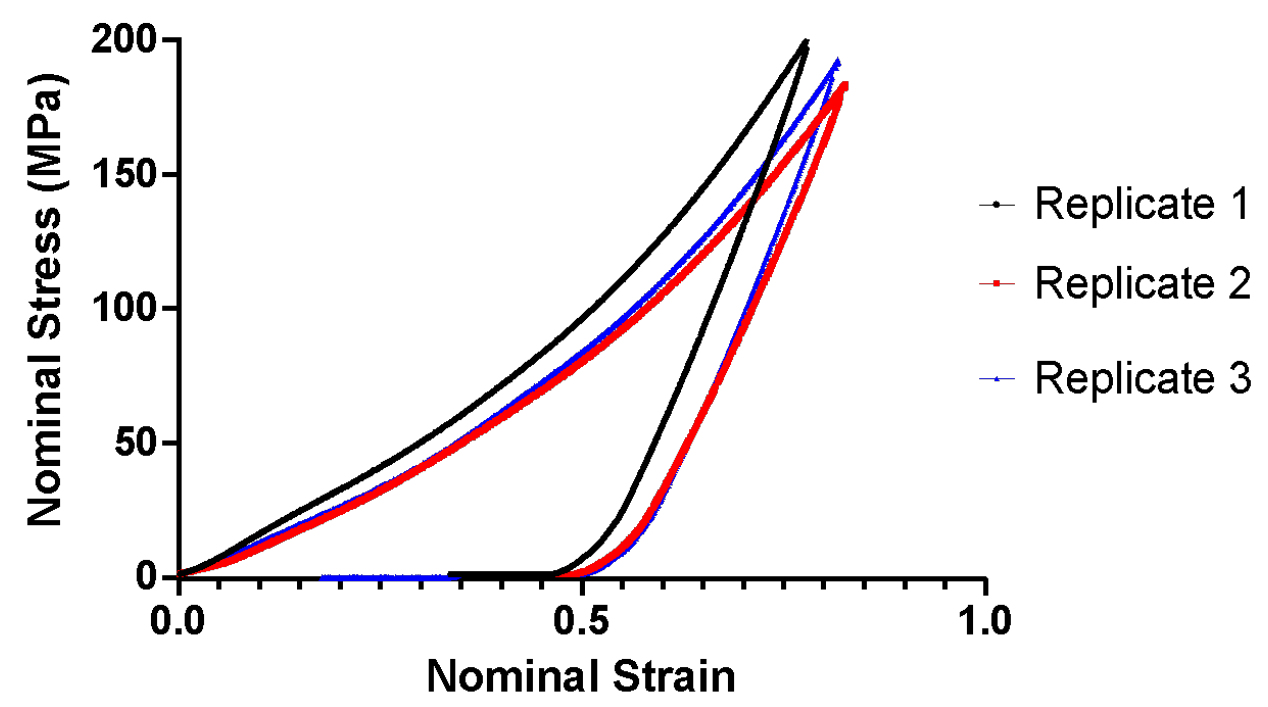

Figure S28. Compressive stress-strain curve for synthetic sporopollenin analogue 13 
Stress vs. Strain

Synthetic Sporopollenin 14

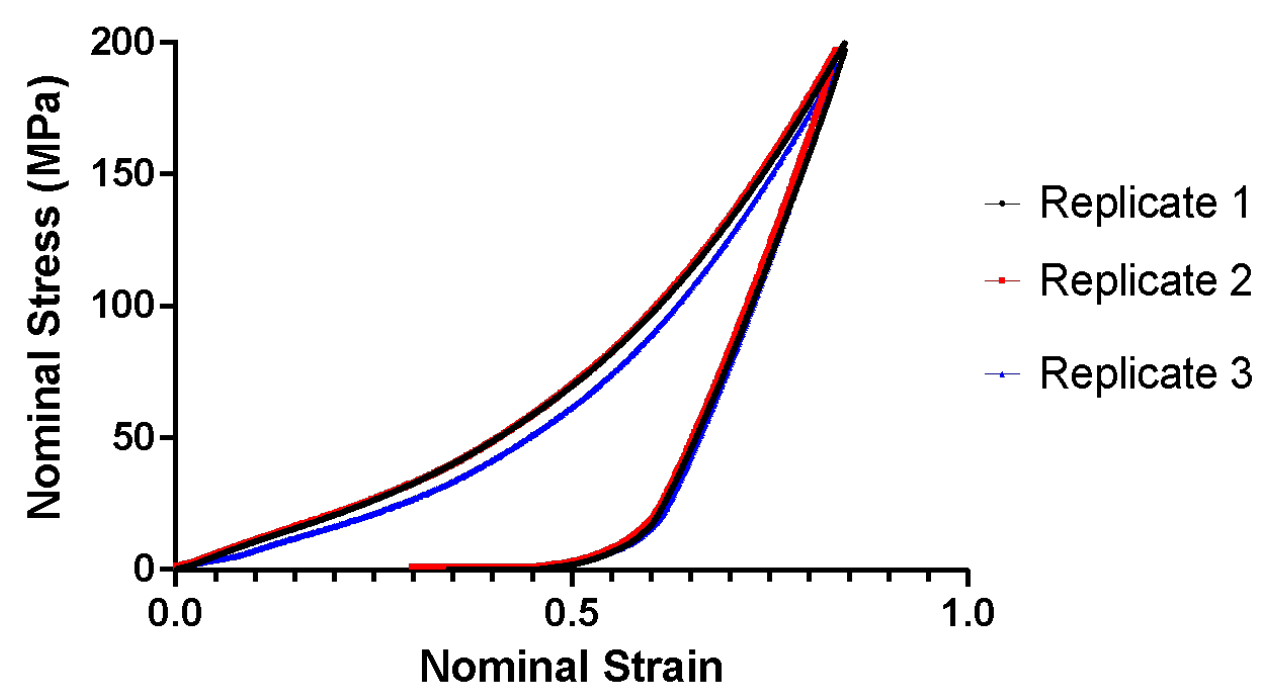

Figure S29. Compressive stress-strain curve for synthetic sporopollenin analogue 14

Stress vs. Strain

Synthetic Sporopollenin 15

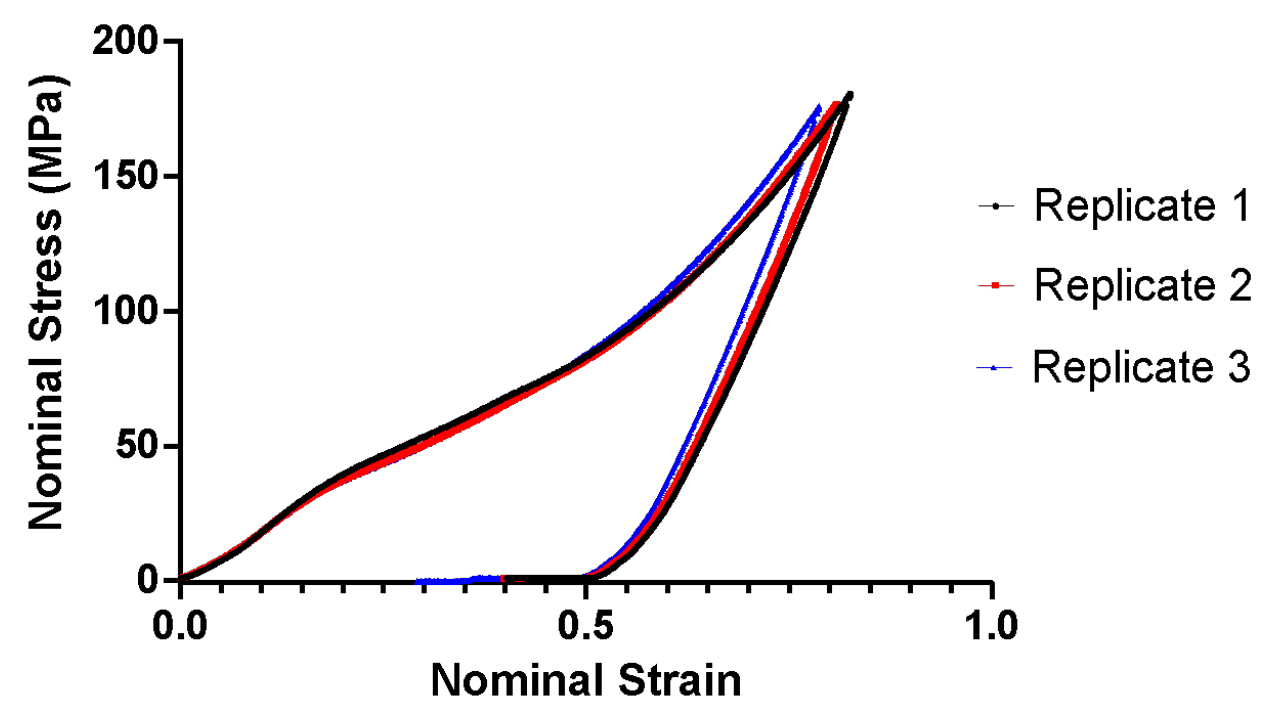

Figure S30. Compressive stress-strain curve for synthetic sporopollenin analogue $\mathbf{1 5}$ 


\section{Stress vs. Strain}

Synthetic Sporopollenin 16

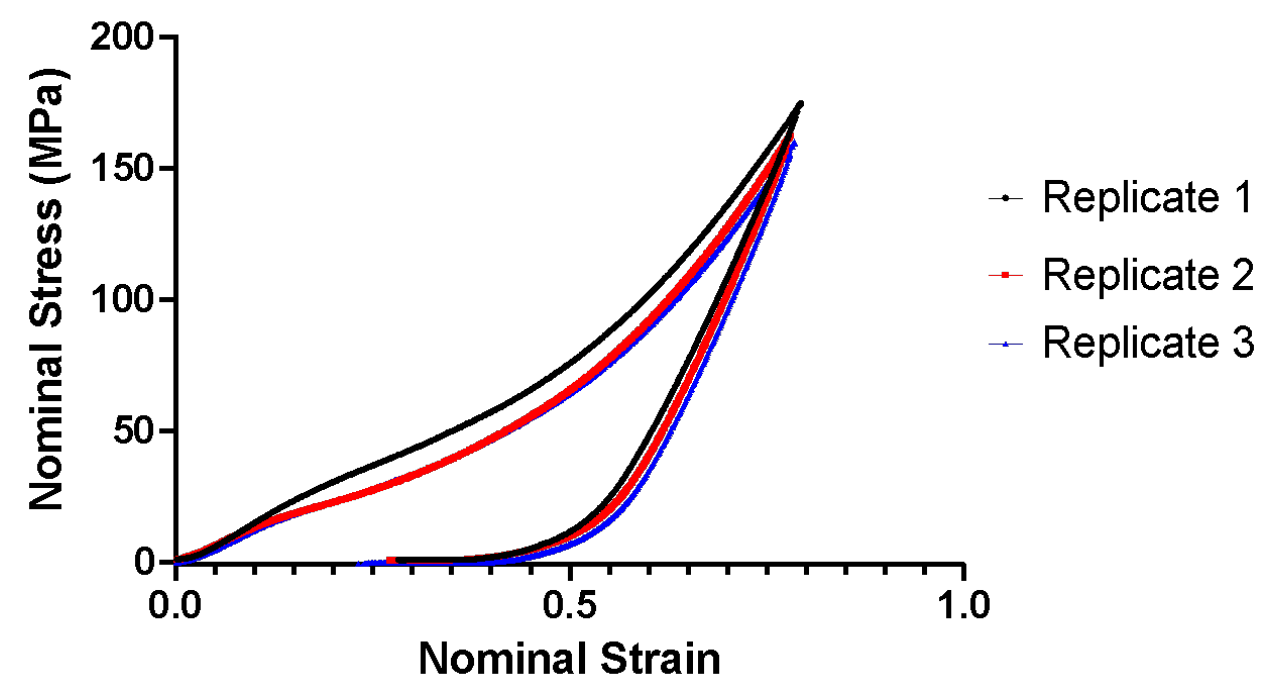

Figure S31. Compressive stress-strain curve for synthetic sporopollenin analogue 16

Stress vs. Strain

Synthetic Sporopollenin 17

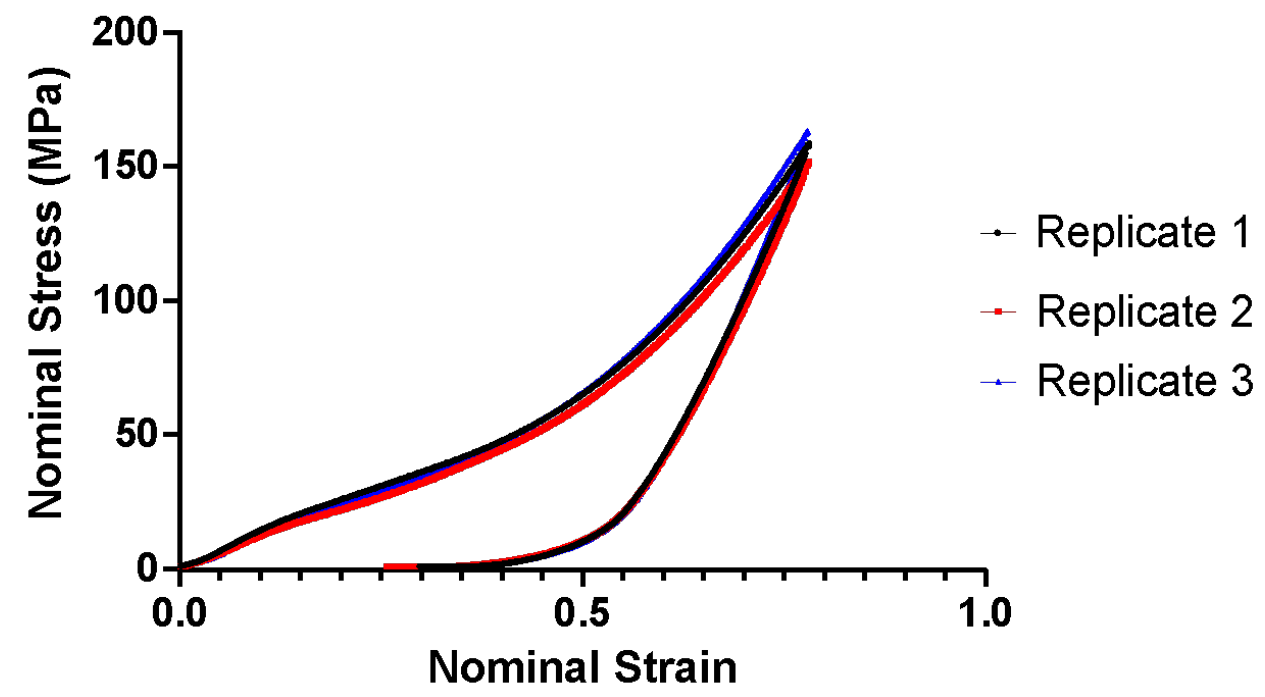

Figure S32. Compressive stress-strain curve for synthetic sporopollenin analogue 17 


\section{Stress vs. Strain}

Synthetic Sporopollenin 18

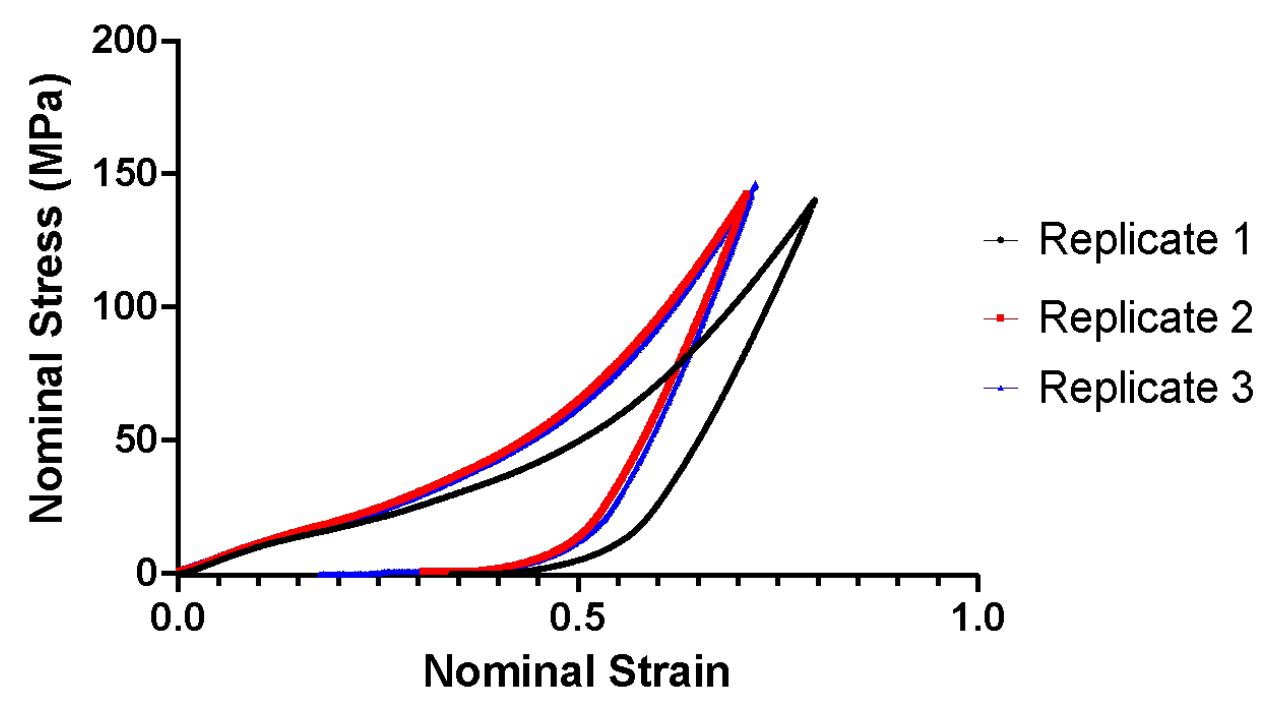

Figure S33. Compressive stress-strain curve for synthetic sporopollenin analogue 18

\section{Stress vs. Strain}

Synthetic Sporopollenin 26

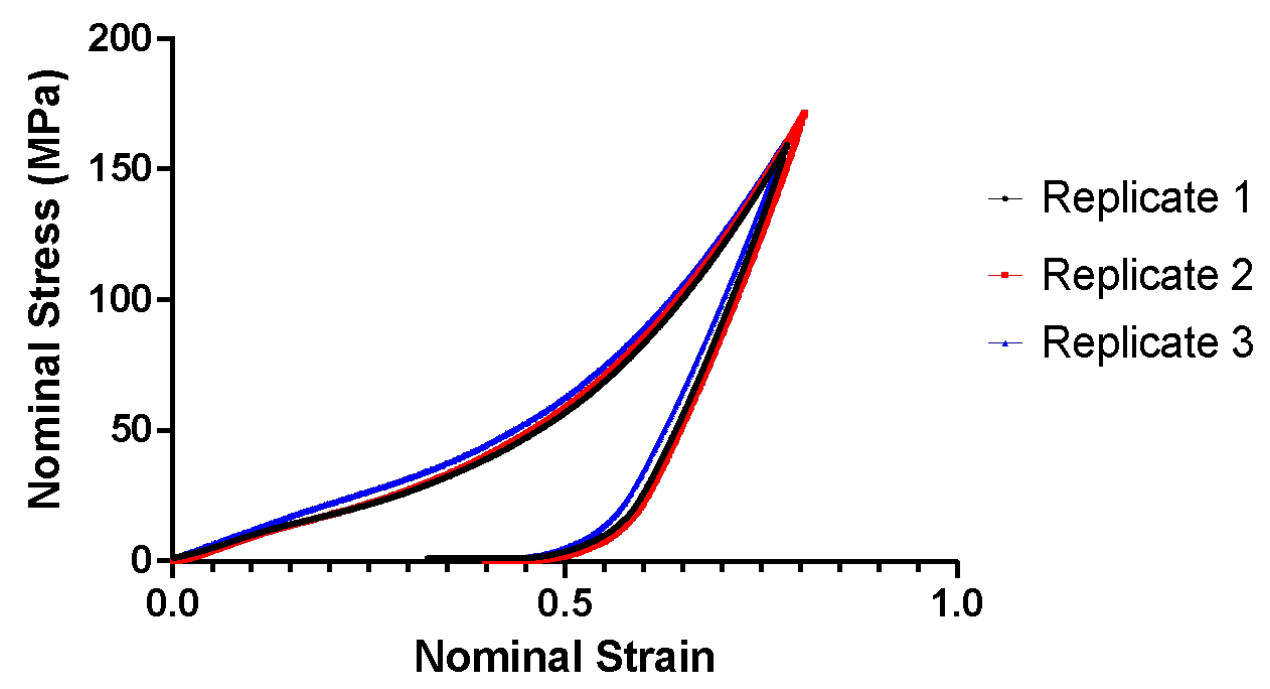

Figure S34. Compressive stress-strain curve for synthetic sporopollenin analogue 26 


\section{Modulus vs. Linker Carbon Count}

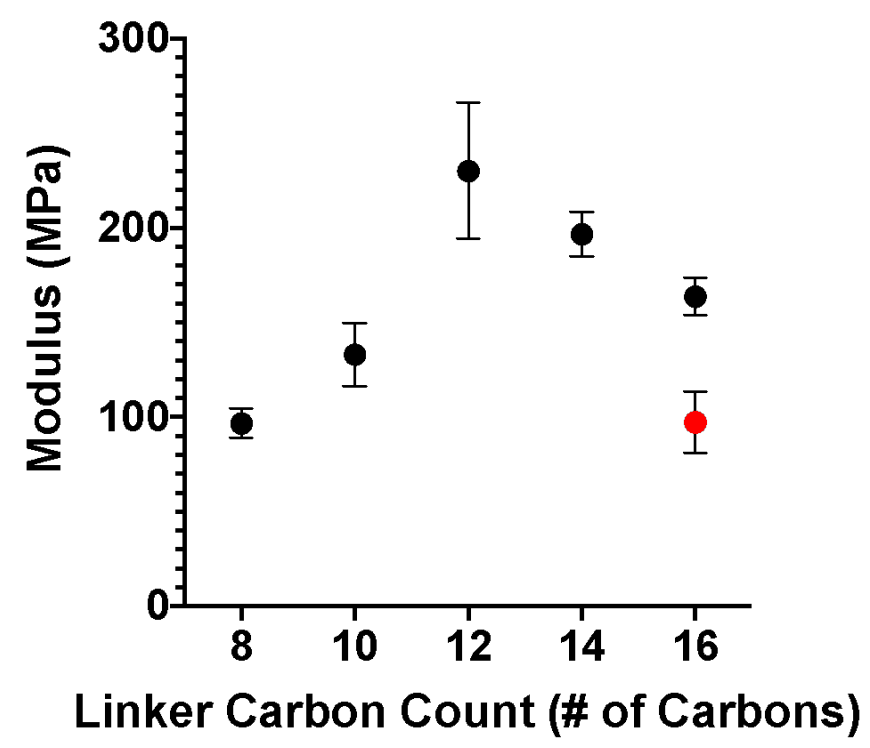

Figure S35. Relationship between compressive modulus and linker carbon count in simplified (black) and substituted (red) synthetic linker sporopollenin analogues

\section{Modulus vs. Linker Density}

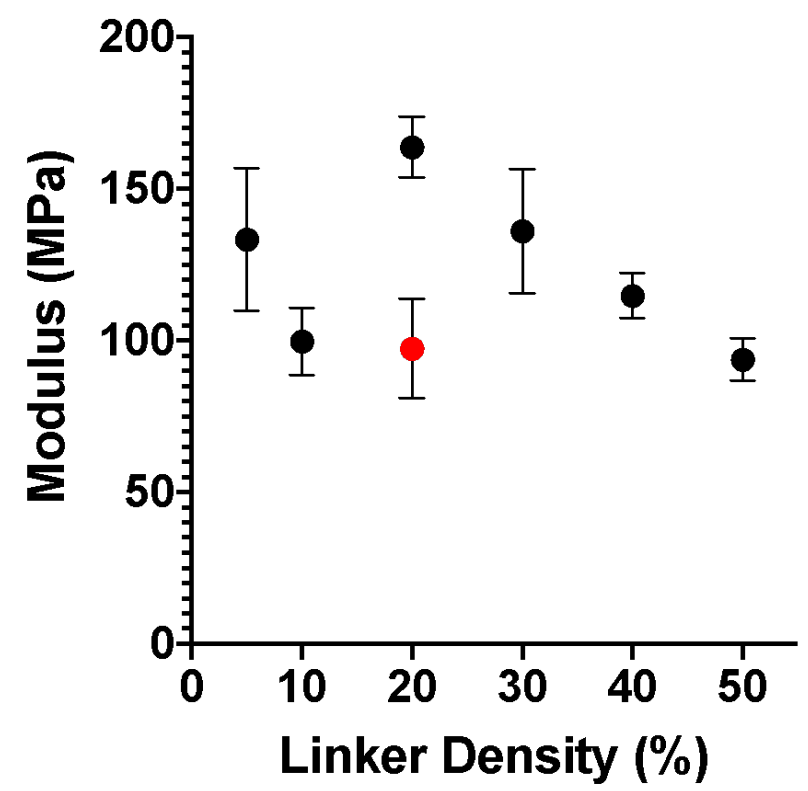

Figure S36. Relationship between compressive modulus and linker density in simplified (black) and substituted (red) synthetic linker sporopollenin analogues 


\section{Modulus vs. PVA Avg. MW}

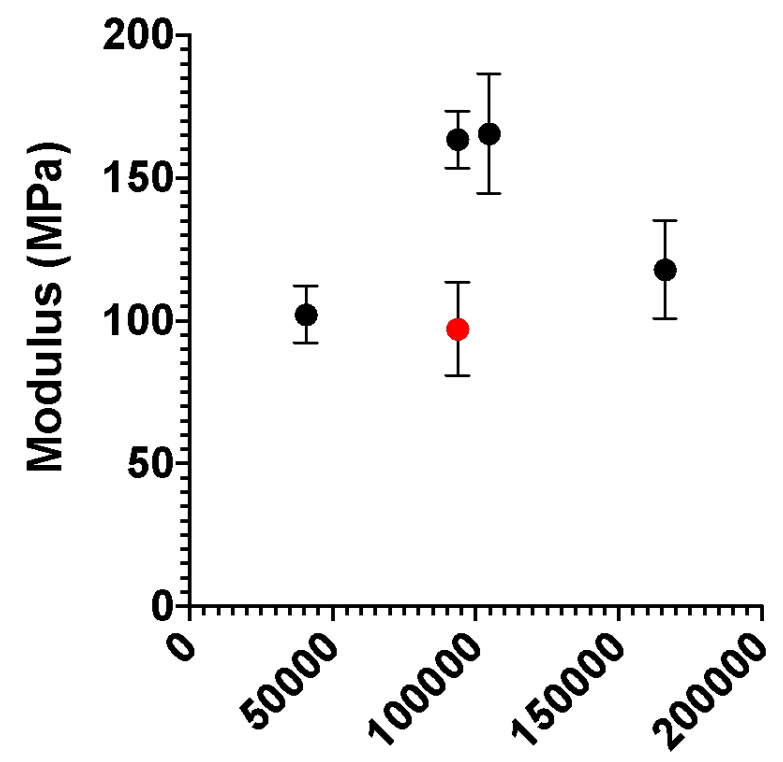

\section{PVA Average Molecular Weight $(\mathrm{g} / \mathrm{mol})$}

Figure S37. Relationship between compressive modulus and PVA $\mathrm{MW}_{\text {avg }}$ in simplified (black) and substituted (red) synthetic linker sporopollenin analogues

\section{Hysteresis Ratio vs. Linker Carbon Count}

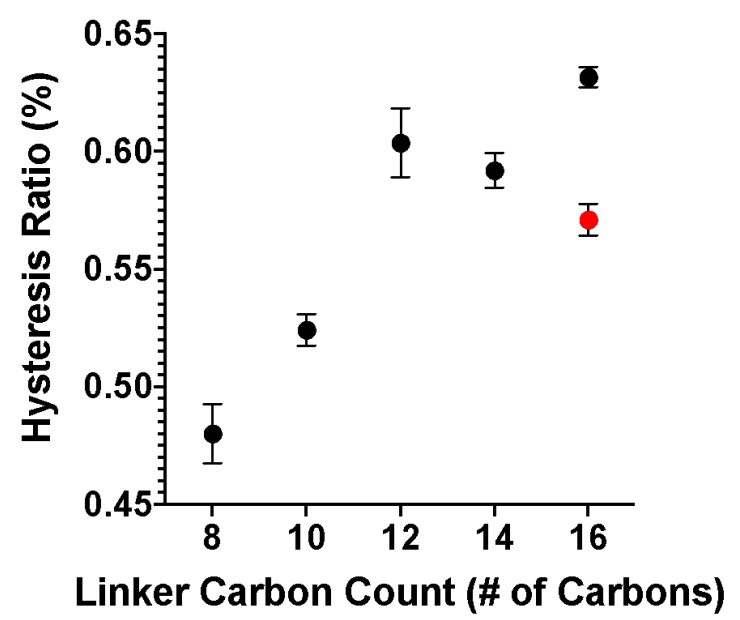

Figure S38. Relationship between compressive hysteresis ratio and linker carbon count in simplified (black) and substituted (red) synthetic linker sporopollenin analogues 


\section{Hysteresis Ratio vs. Linker Density}

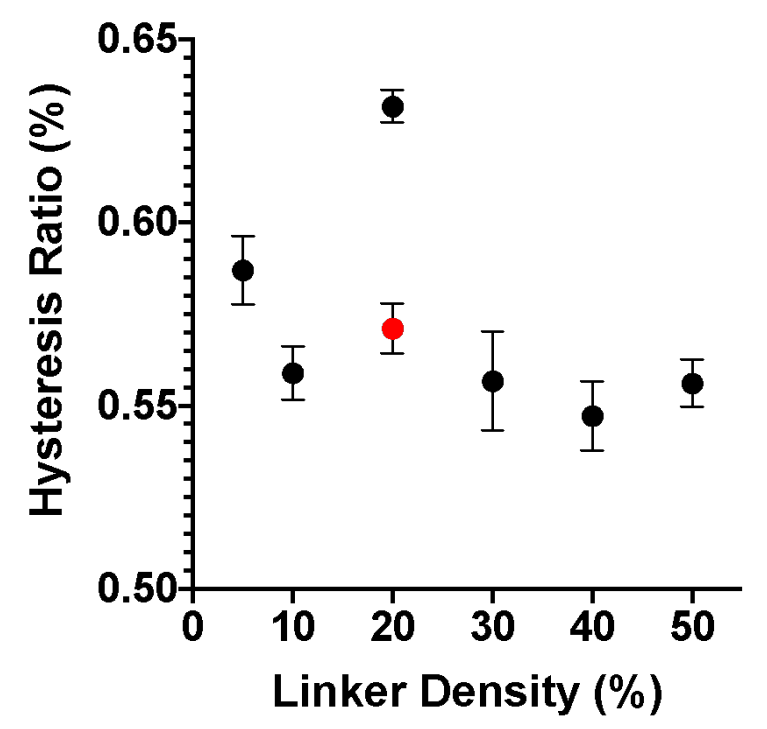

Figure S39. Relationship between compressive hysteresis ratio and linker density in simplified (black) and substituted (red) synthetic linker sporopollenin analogues

\section{Hysteresis Ratio vs. PVA Avg. MW}

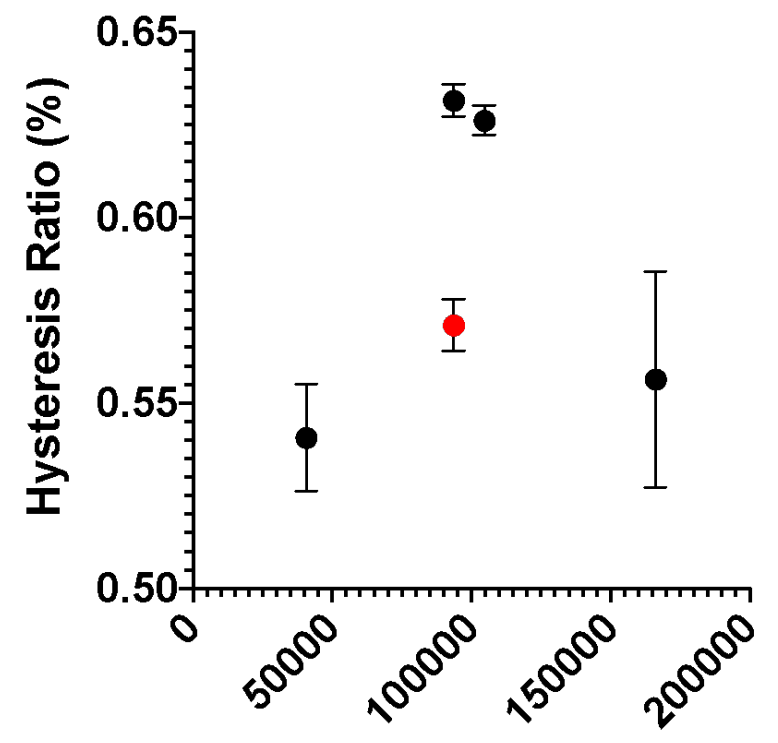

PVA Average Molecular Weight $(\mathrm{g} / \mathrm{mol})$

Figure S40. Relationship between compressive hysteresis ratio and PVA MW avg in simplified (black) and substituted (red) synthetic linker sporopollenin analogues 
Stress vs. Stretch

Rate Dependence, Tensile

Synthetic Sporopollenin 12

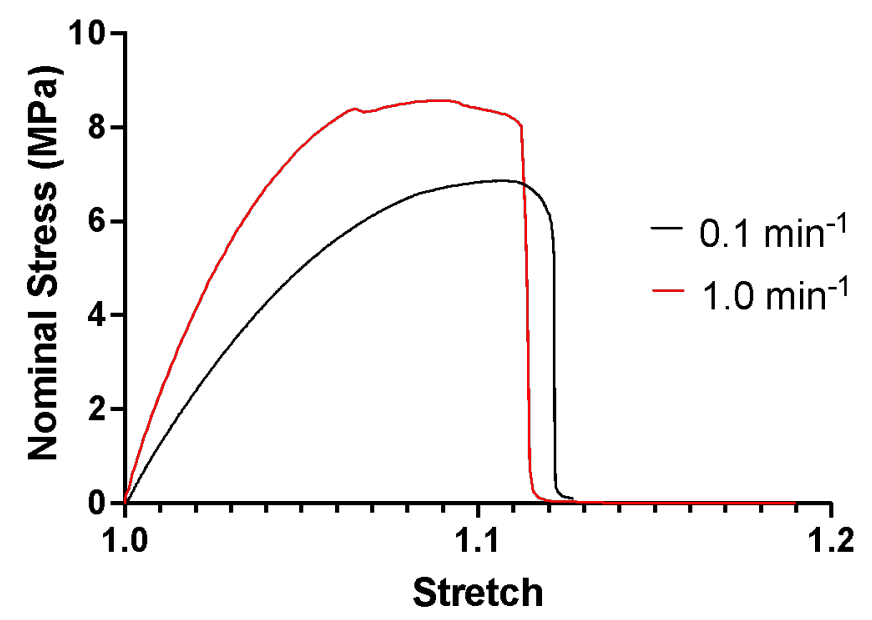

Figure S41. Tensile stress-strain curve for synthetic sporopollenin analogue 12 at 1.0 (red) and $0.1 \mathrm{~min}^{-1}$ (black) loading rates

\section{Stress vs. Stretch Loading Cycles, Tensile Synthetic Sporopollenin 12}

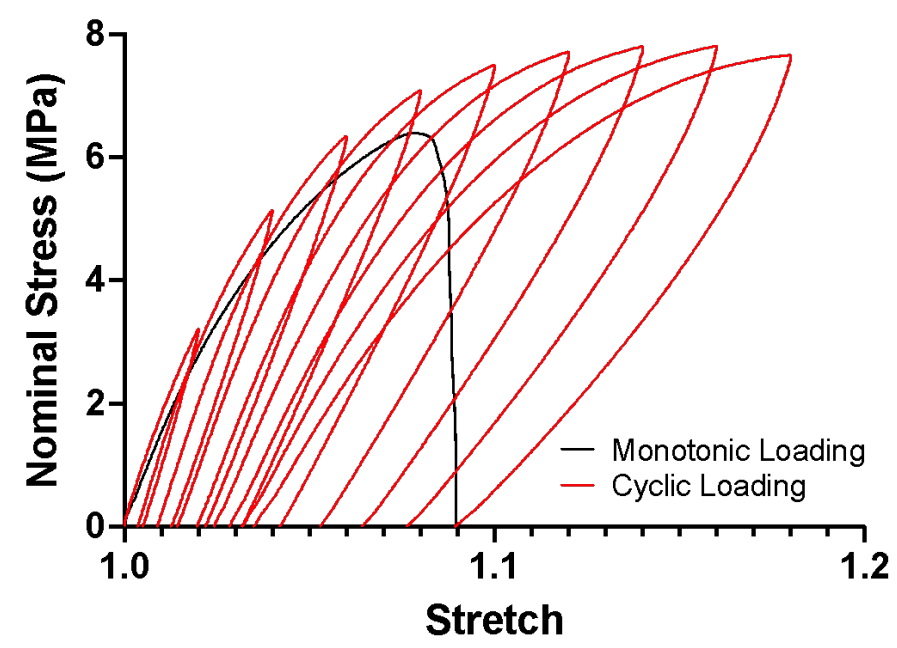

Figure S42. Tensile stress-strain curve for synthetic sporopollenin analogue 12 with cyclic (red) and monotonic (black) loading 


\section{Stress vs. Time \\ Relaxation, Tensile \\ Synthetic Sporopollenin 12}

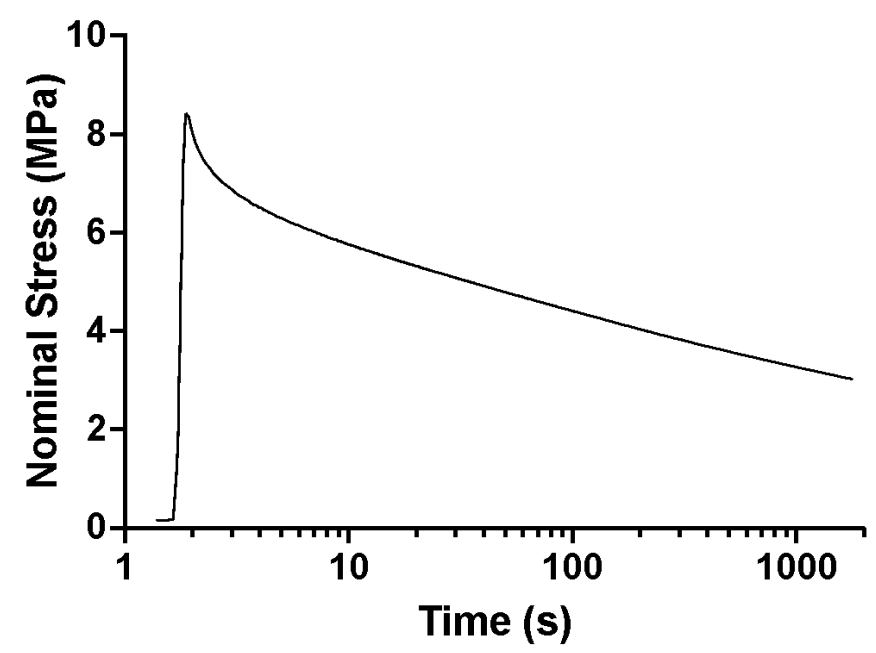

Figure S43. Tensile stress-time relaxation curve for synthetic sporopollenin analogue $\mathbf{1 2}$

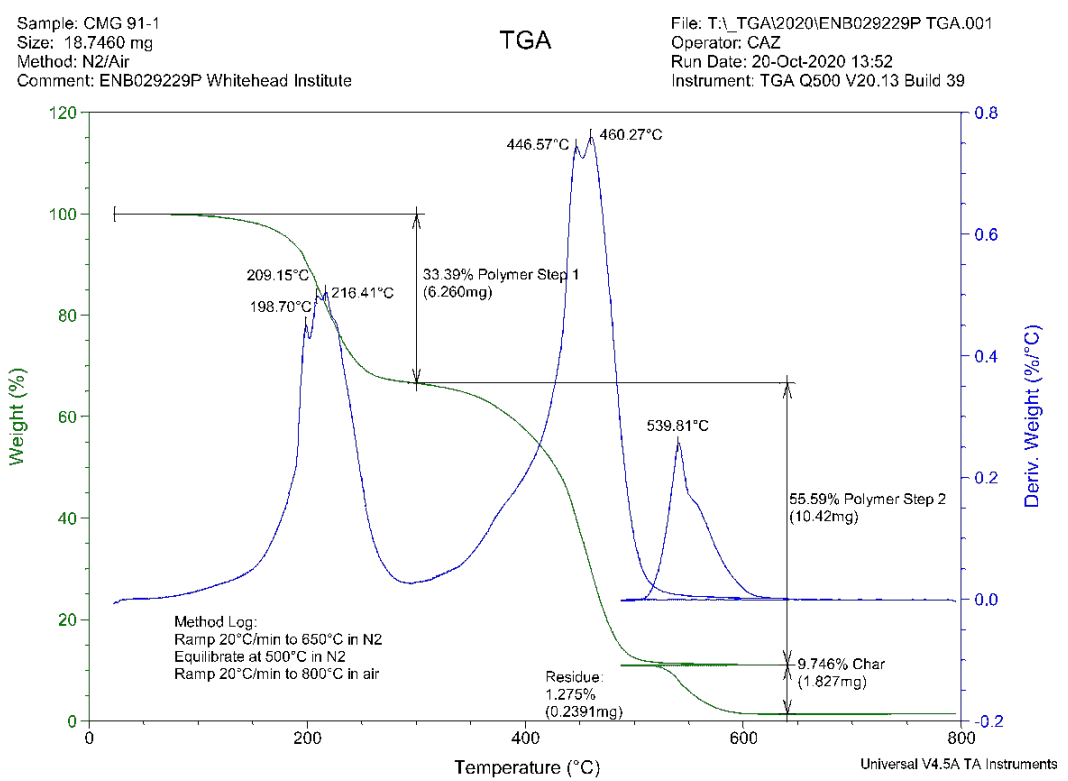

Figure S44. The TGA thermogram for synthetic sporopollenin analogue 6 


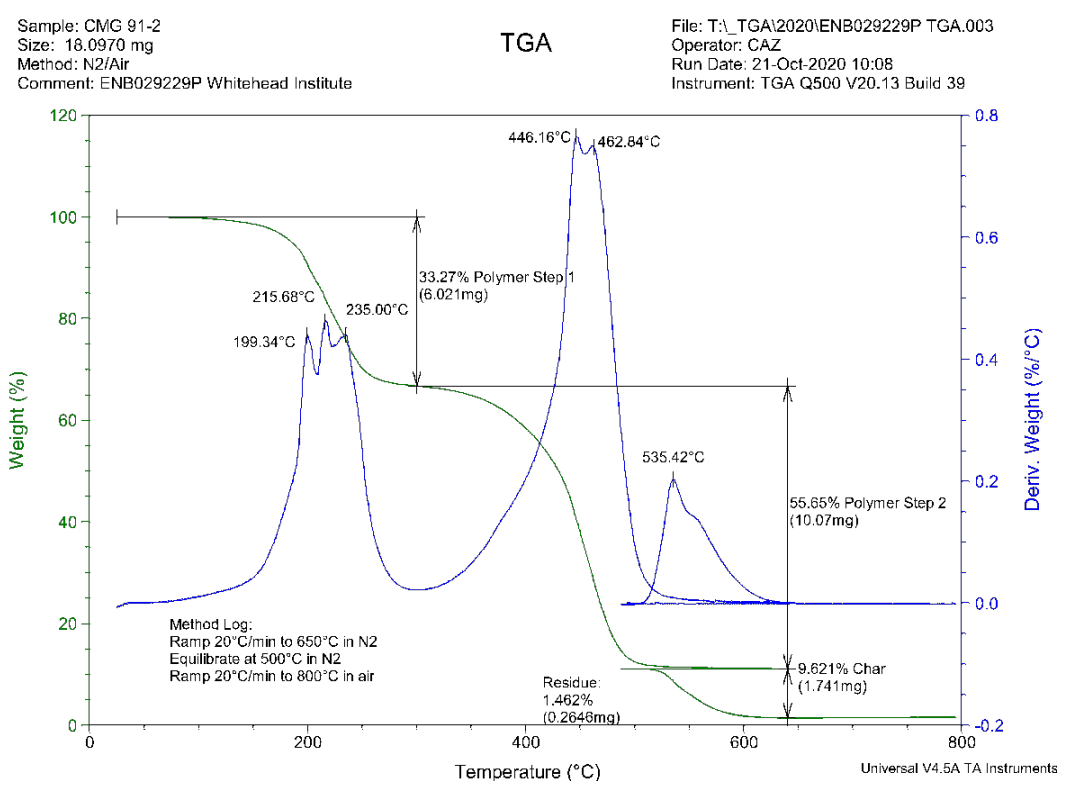

Figure S45. The TGA thermogram for synthetic sporopollenin analogue 7

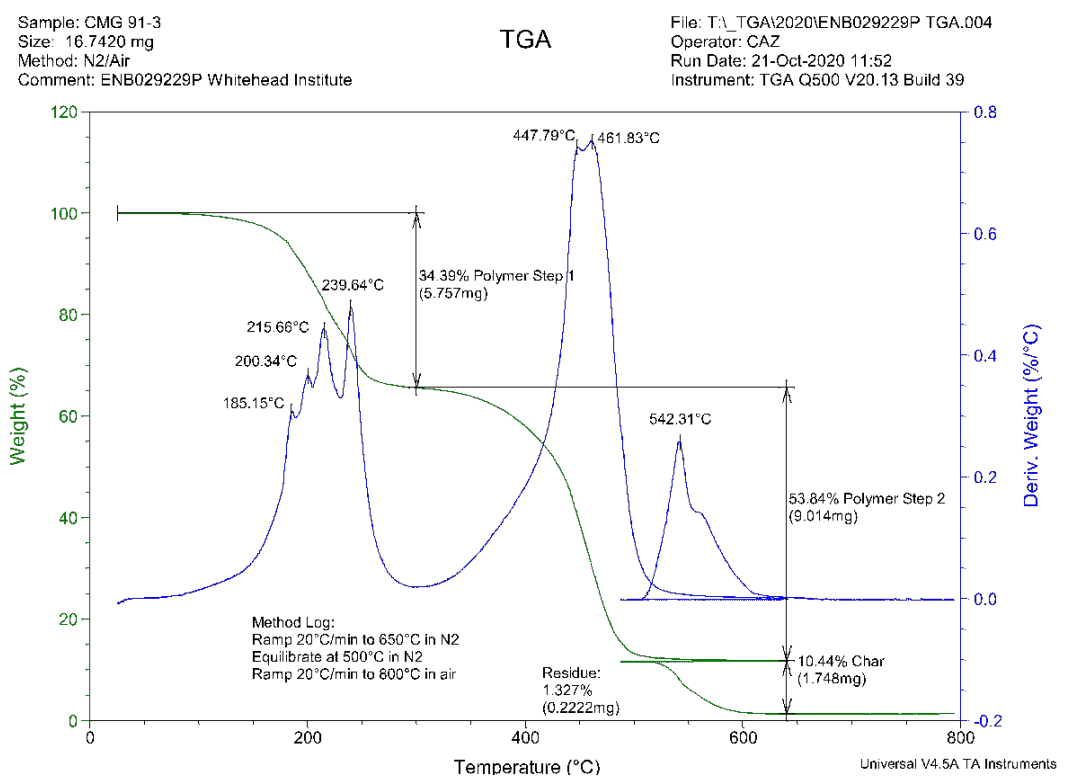

Figure S46. The TGA thermogram for synthetic sporopollenin analogue $\mathbf{8}$ 


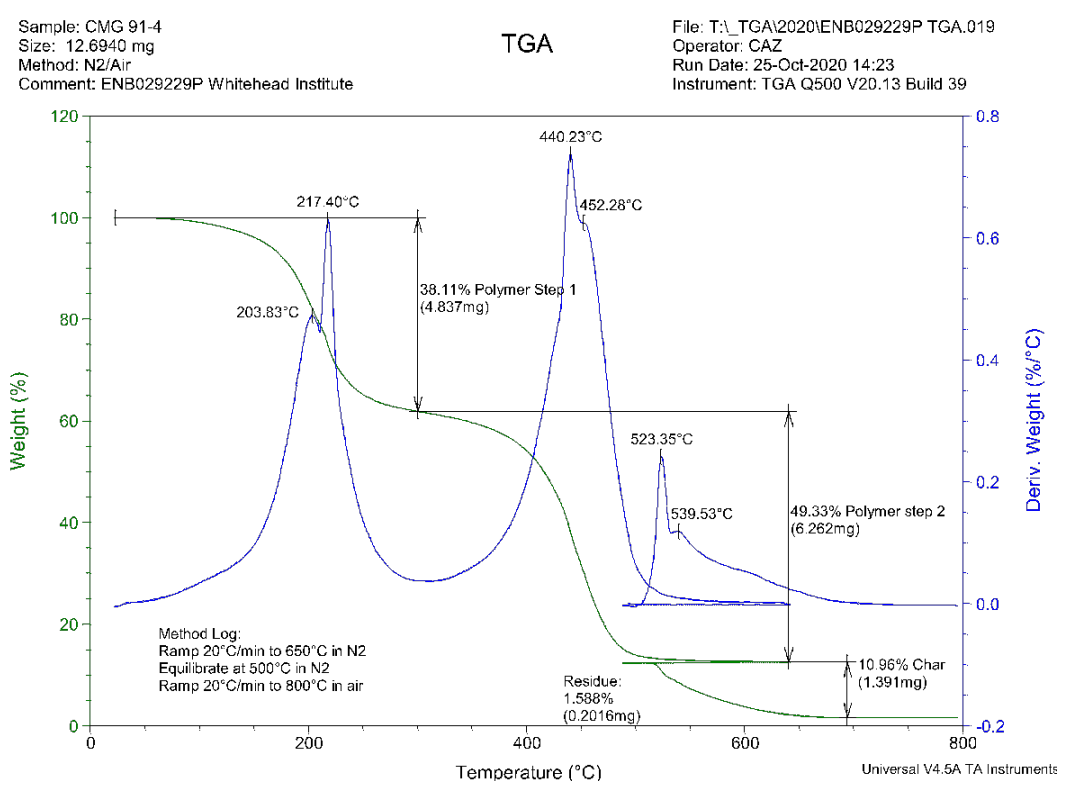

Figure S47. The TGA thermogram for synthetic sporopollenin analogue 9

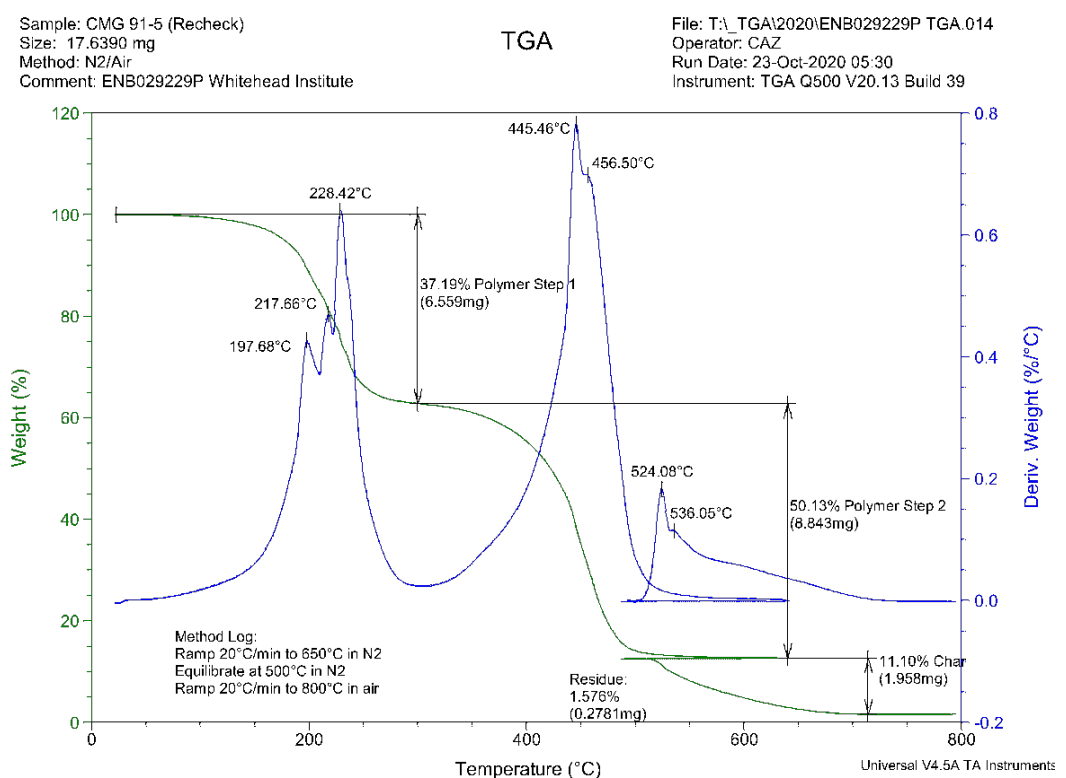

Figure S48. The TGA thermogram for synthetic sporopollenin analogue $\mathbf{1 0}$ 


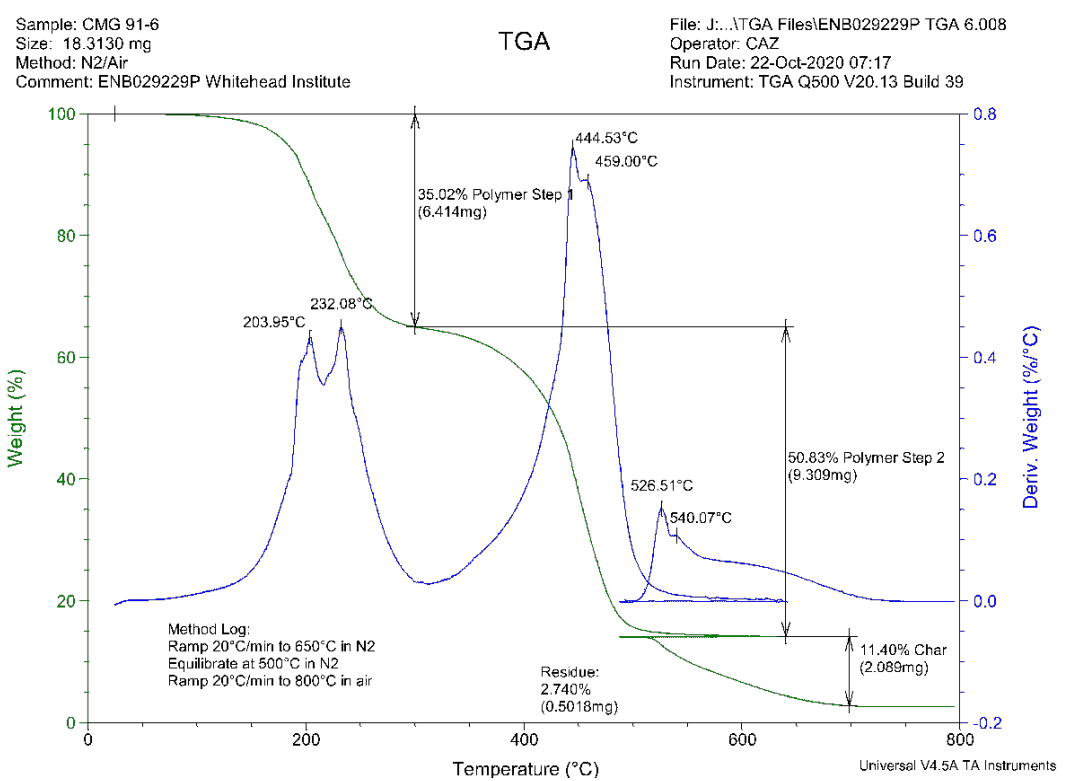

Figure S49. The TGA thermogram for synthetic sporopollenin analogue 11

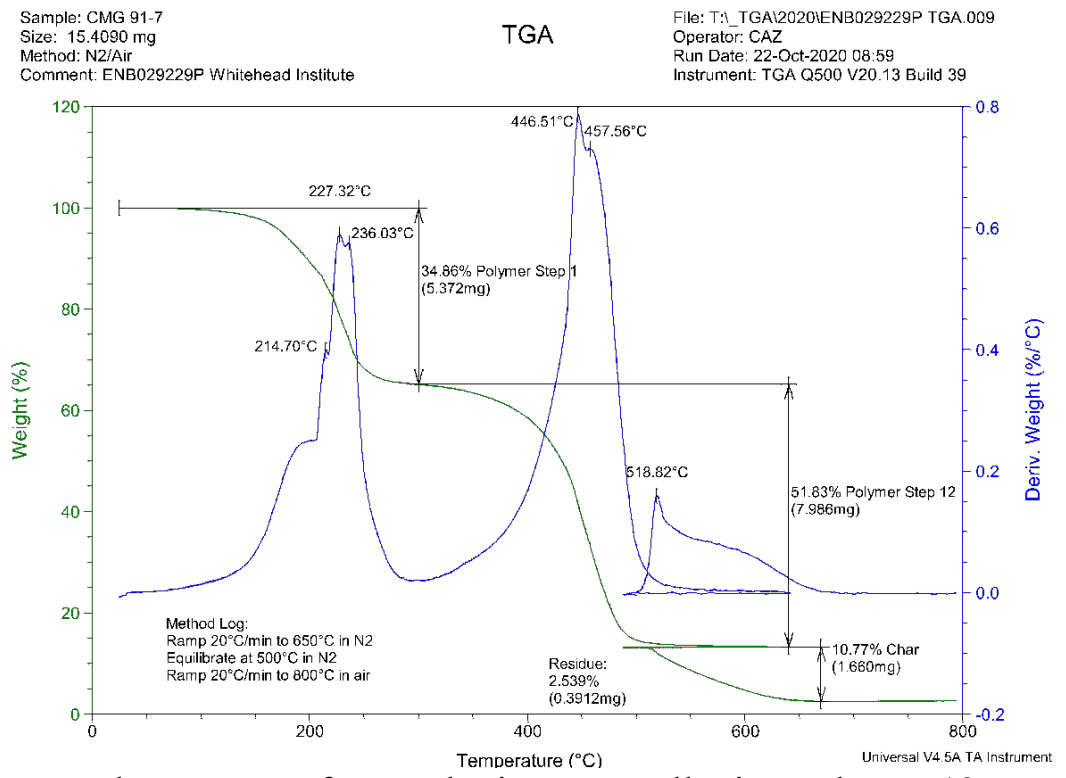

Figure S50. The TGA thermogram for synthetic sporopollenin analogue $\mathbf{1 2}$ 


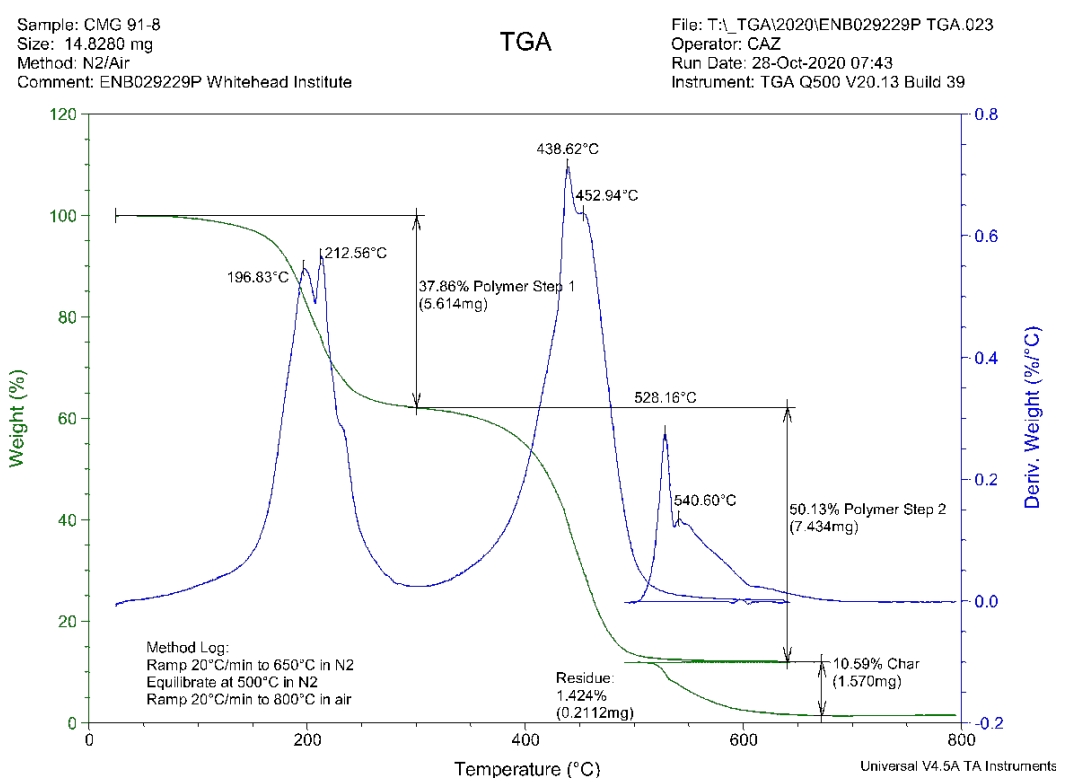

Figure S51. The TGA thermogram for synthetic sporopollenin analogue $\mathbf{1 3}$

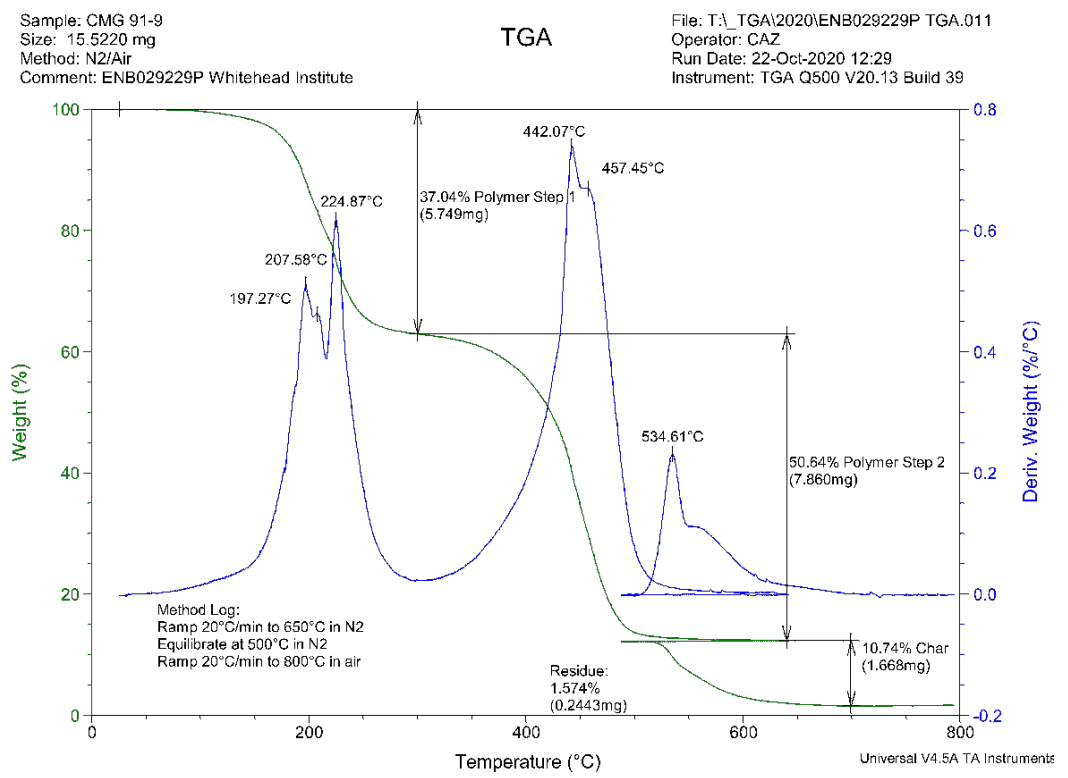

Figure S52. The TGA thermogram for synthetic sporopollenin analogue 14 


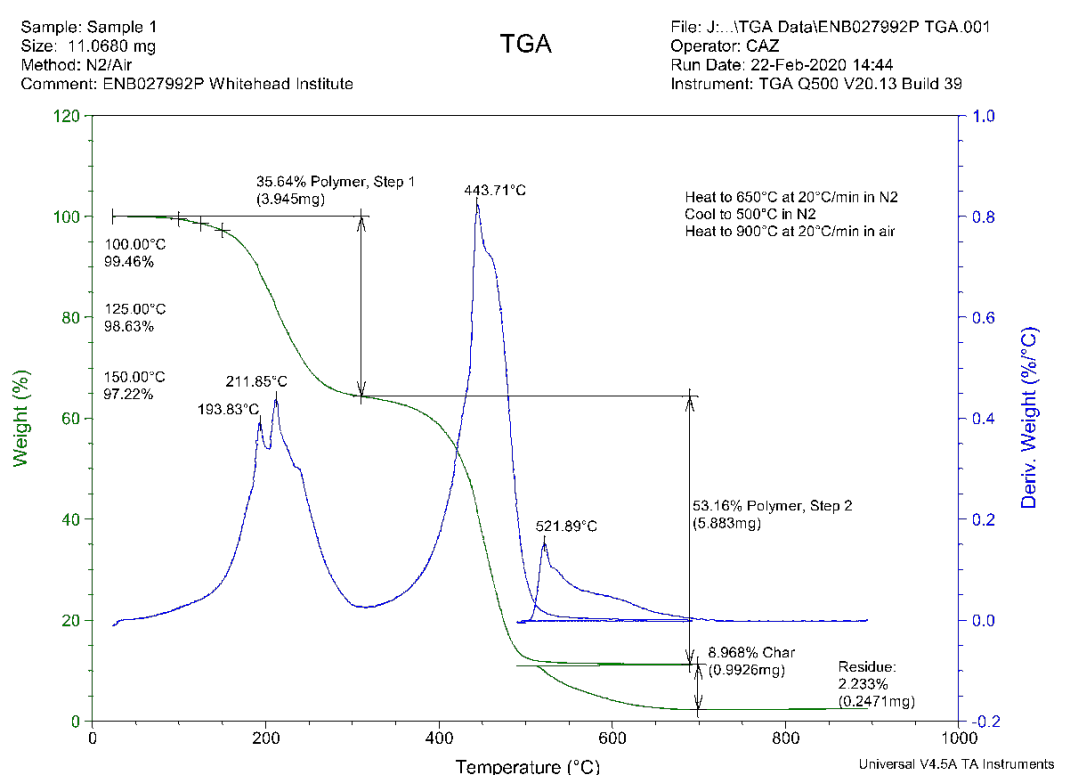

Figure S53. The TGA thermogram for synthetic sporopollenin analogue $\mathbf{1 5}$



Figure S54. The TGA thermogram for synthetic sporopollenin analogue 16 


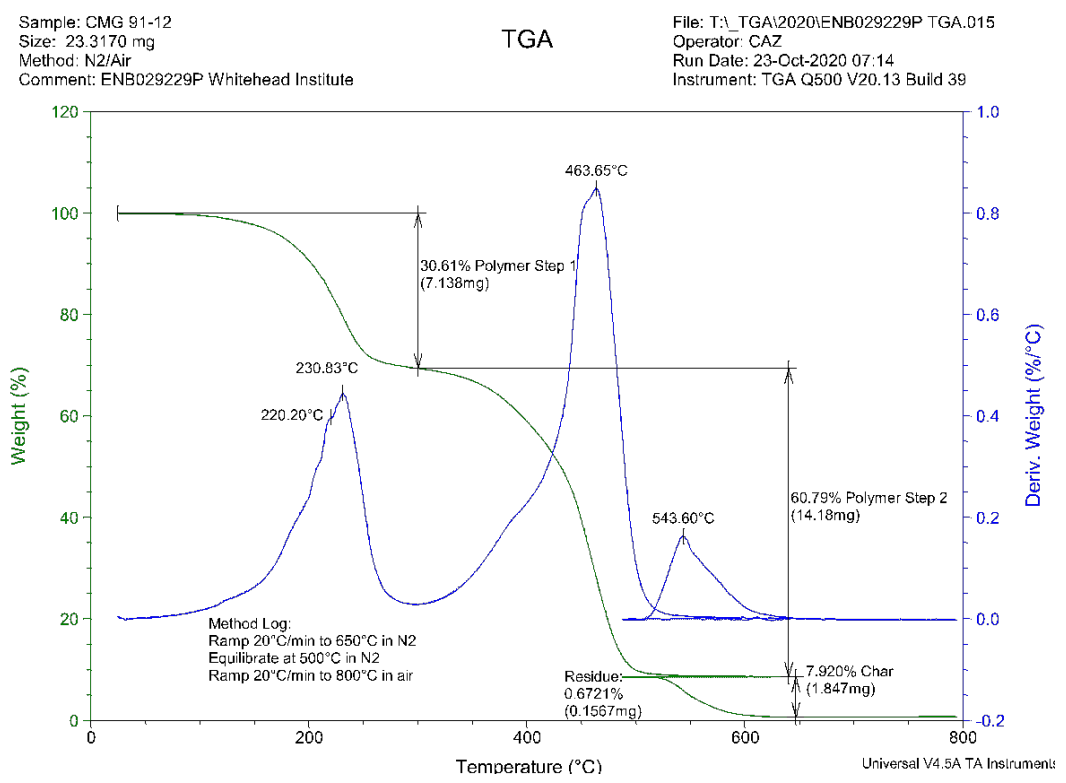

Figure S55. The TGA thermogram for synthetic sporopollenin analogue 17

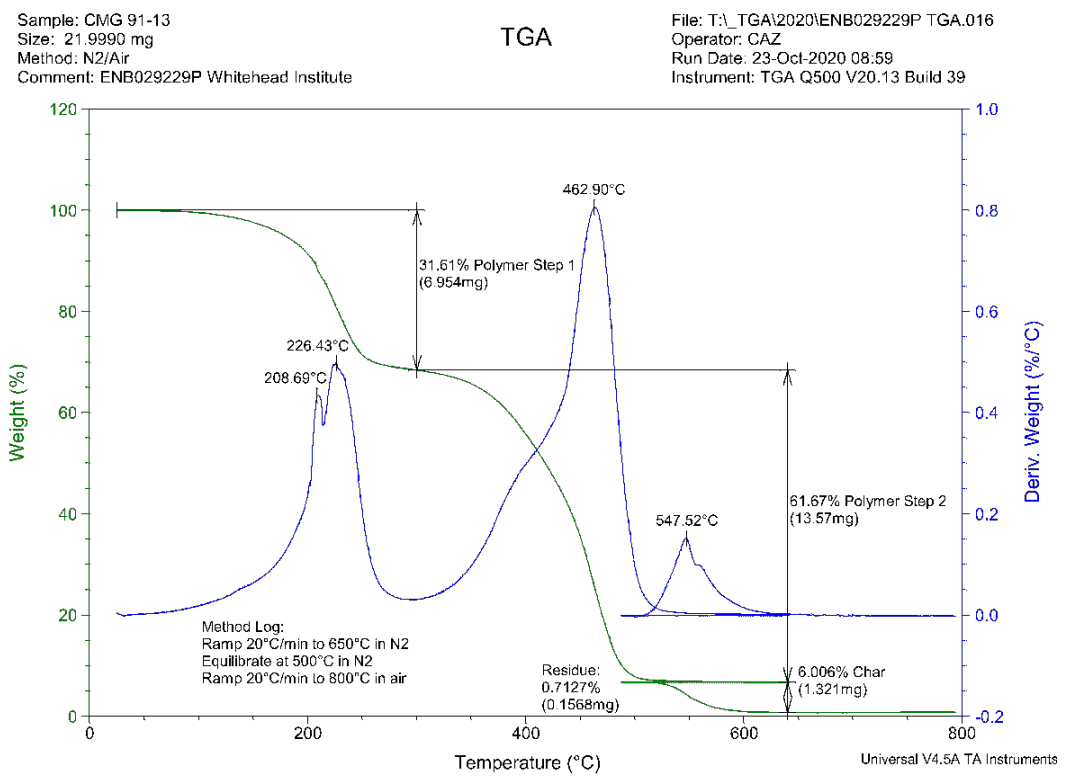

Figure S56. The TGA thermogram for synthetic sporopollenin analogue 18 


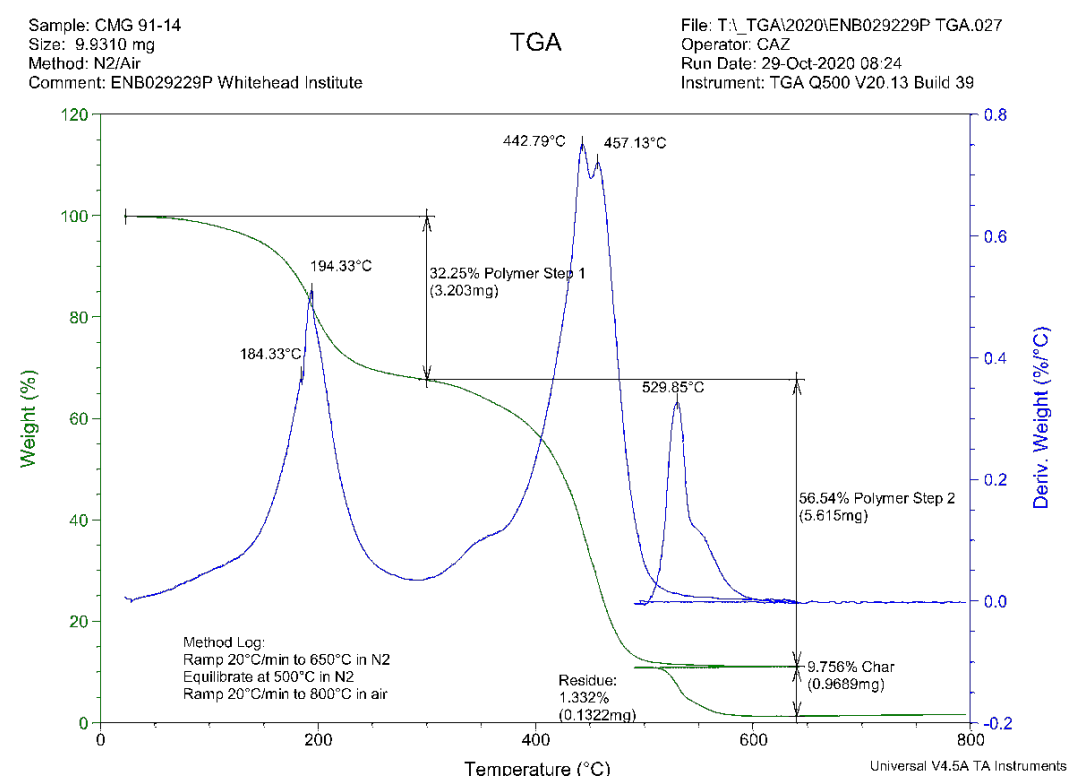

Figure S57. The TGA thermogram for synthetic sporopollenin analogue 26

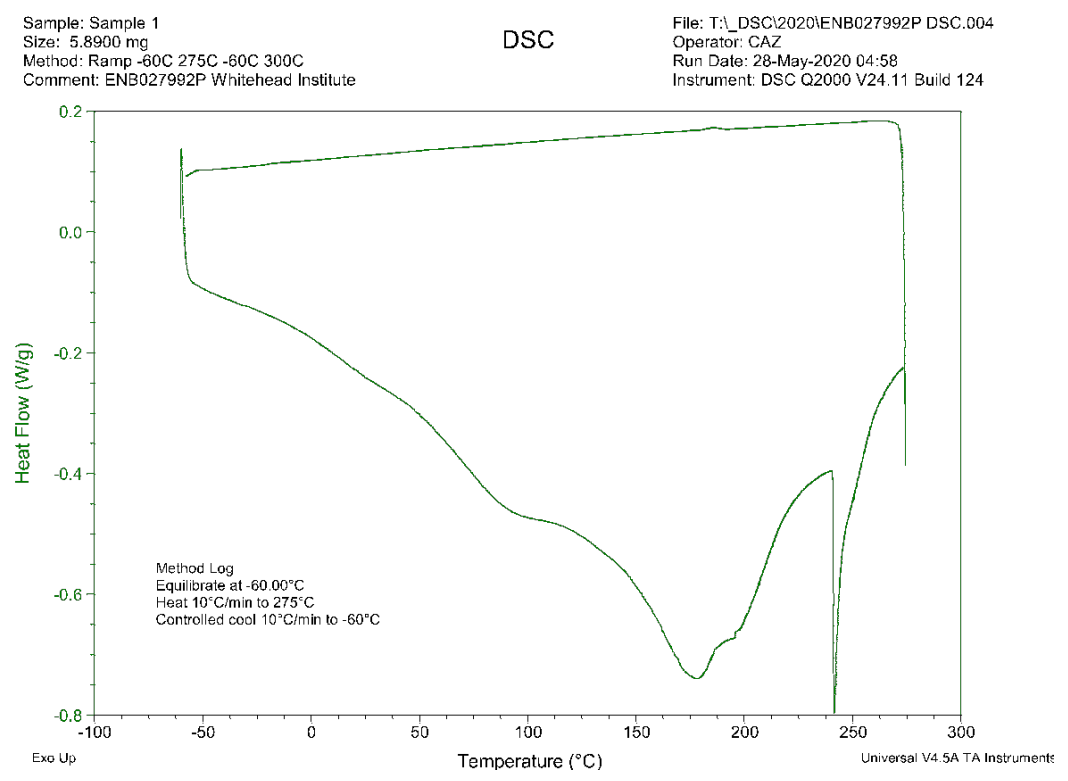

Figure S58. The DSC thermogram for synthetic sporopollenin analogue 15, first replicate 


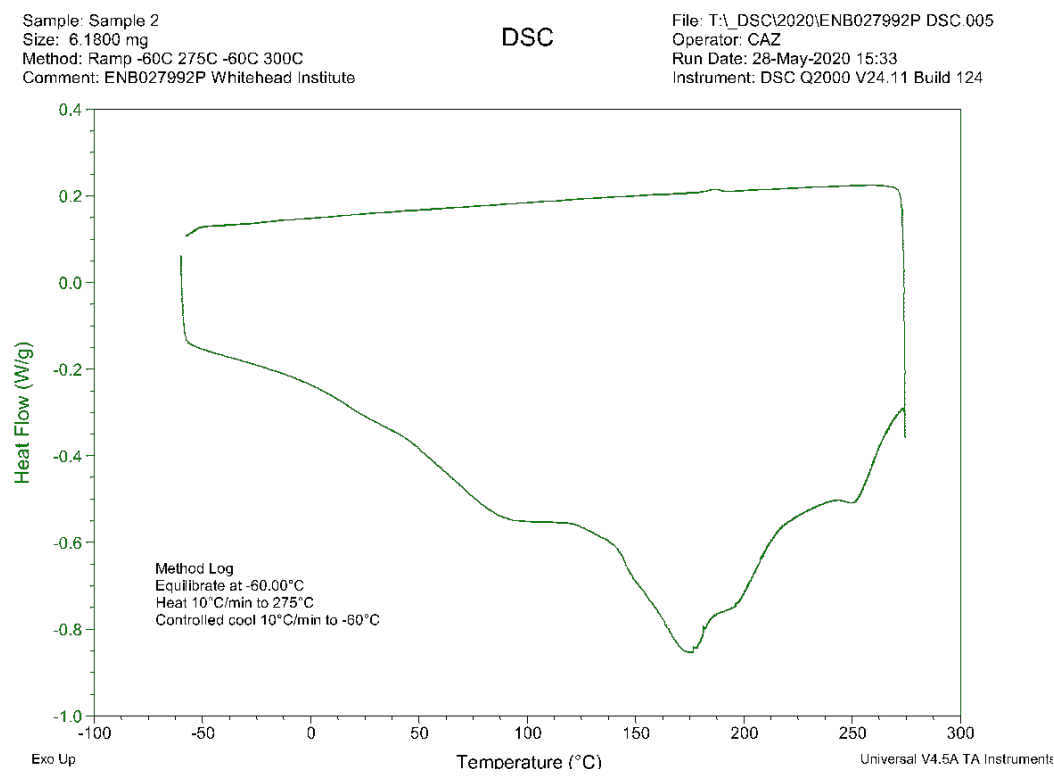

Figure S59. The DSC thermogram for synthetic sporopollenin analogue 15, second replicate

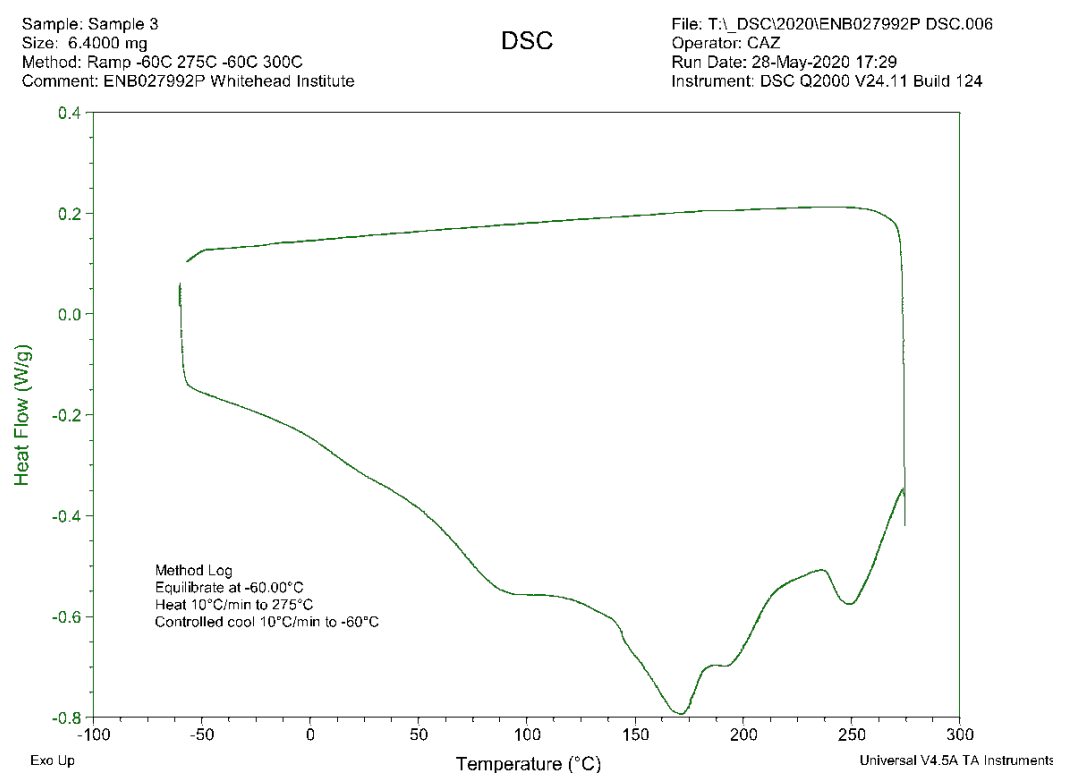

Figure S60. The DSC thermogram for synthetic sporopollenin analogue 15, third replicate 


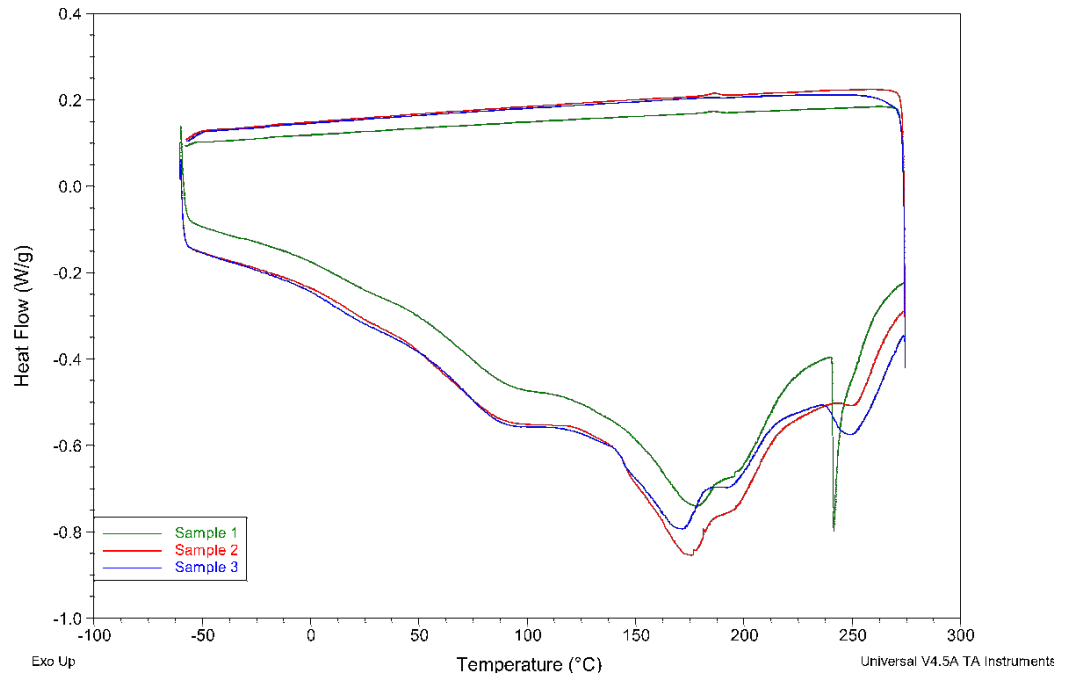

Figure S61. The DSC thermogram for synthetic sporopollenin analogue 15, all replicates

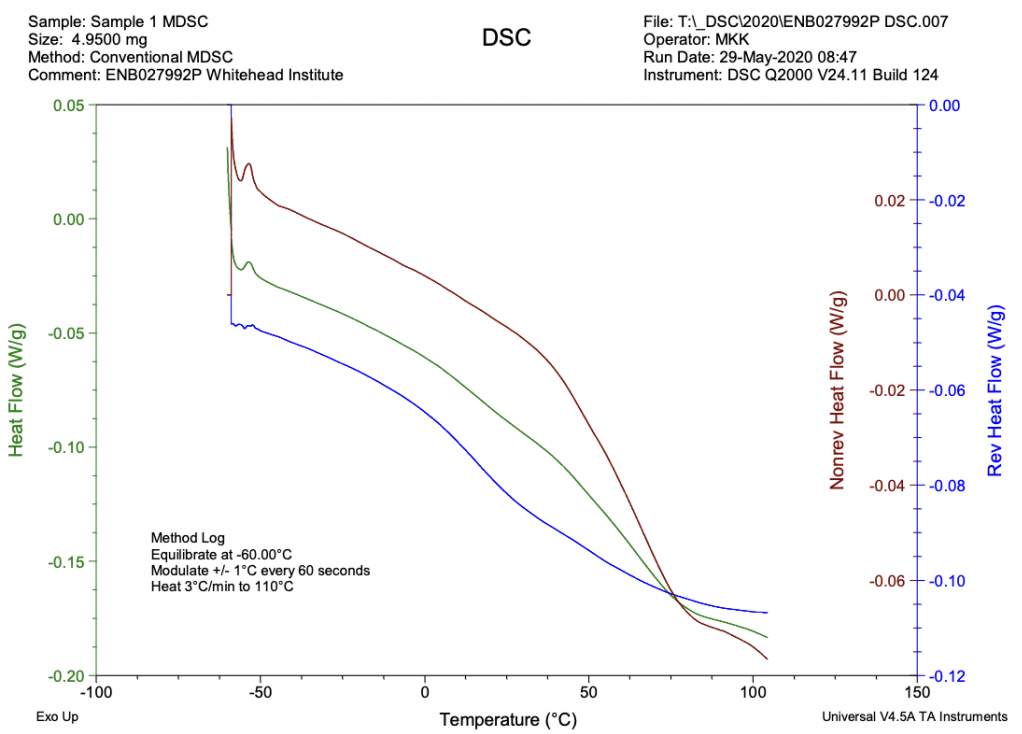

Figure S62. The modulated DSC thermogram for synthetic sporopollenin analogue $\mathbf{1 5}$ 


\section{References}

1. Roels, J.; Metz, P. Synlett 2001, 789.

2. Mangaleswaran, S.; Argade, N. P. J. Org. Chem. 2001, 66, 5259.

3. Ziffle, V. E.; Cheng, P.; Clive, D. L. J. J. Org. Chem. 2010, 75, 8024.

4. Degani, I.; Fochi, R. J. Chem. Soc. Perkin Trans. 1 1976, 323.

5. Guillonneau, L; Taddei, D.; Moody, C. J. Org. Lett. 2008, 10, 4505.

6. Takezawa, E.; Sakaguchi, S.; Ishii, Y. Org. Lett. 1999, 1, 713.

7. Song, Y.-Y.; Kinami, K.; Kato, A.; Jia, Y. -M.; Li, Y. -X.; Fleet, G. W. J.; Yu, C. -Y. Org. Biomol. Chem. 2016, 14, 5157.

8. (a) Brandt, D. R.; Pannone, K. M.; Romano, J. J.; Casillas, E. G. Tetrahedron 2013, 69, 9994.

(b) Liu, S. X.; Jin, J. Z.; Shan, L.; Zeng, H. W.; Chen, B. Y.; Sun, Q. Y.; Zhang, W. D. Bioorg. Med. Chem. Lett. 2013, 23, 2207.

9. Li, F. -S.; Phyo, P.; Jacobowitz, J.; Hong, M.; Weng, J. -K. Nat. Plants 2019, 5, 41. 